OPEN ACCESS

Edited by: Dayang Wang,

RMIT University, Australia

Reviewed by:

Haibing Xia,

Shandong University, China

Ali Abou-Hassan,

Pierre-and-Marie-Curie

University, France

*Correspondence:

P. Davide Cozzoli

davide.cozzoli@unisalento.it

Specialty section: This article was submitted to Colloidal Materials and Interfaces, a section of the journal Frontiers in Materials

Received: 06 September 2016 Accepted: 05 December 2016 Published: 20 December 2016

Citation:

Scarfiello R, Nobile C and Cozzoli PD (2016) Colloidal Magnetic Heterostructured Nanocrystals with Asymmetric Topologies: Seeded-Growth Synthetic Routes and Formation Mechanisms.

Front. Mater. 3:56

doi: 10.3389/fmats.2016.00056

\section{Colloidal Magnetic Heterostructured Nanocrystals with Asymmetric Topologies: Seeded-Growth Synthetic Routes and Formation Mechanisms}

\author{
Riccardo Scarfiello ${ }^{1,2}$, Concetta Nobile ${ }^{1}$ and P. Davide Cozzoli ${ }^{1,2,3 *}$ \\ ${ }^{1}$ CNR NANOTEC - Institute of Nanotechnology, Lecce, Italy, ${ }^{2}$ Department of Mathematics and Physics "E. De Giorgi", \\ University of Salento, Lecce, Italy, ${ }^{3}$ Consorzio INSTM, Firenze, Italy
}

Colloidal inorganic nanocrystals (NCs), free-standing crystalline nanostructures generated and processed in solution phase, represent an important class of advanced nanoscale materials owing to the flexibility with which their physical-chemical properties can be controlled through synthetic tailoring of their compositional, structural, and geometric features and the versatility with which they can be integrated in technological fields as diverse as optoelectronics, energy storage/conversion/production, catalysis, and biomedicine. In recent years, building upon mechanistic knowledge acquired on the thermodynamic and kinetic processes that underlie NC evolution in liquid media, synthetic nanochemistry research has made impressive advances, opening new possibilities for the design, creation, and mastering of increasingly complex "colloidal molecules," in which NC modules of different materials are clustered together via solid-state bonding interfaces into free-standing, easily processable multifunctional nanocomposite systems. This review will provide a glimpse into this fast-growing research field by illustrating progress achieved in the wet-chemical development of last-generation breeds of allinorganic heterostructured nanocrystals (HNCs) in asymmetric non-onionlike geometries, inorganic analogues of polyfunctional organic molecules, in which distinct nanoscale crystalline modules are interconnected in heterodimer, hetero-oligomer, and anisotropic multidomain architectures via epitaxial heterointerfaces of limited extension. The focus will be on modular HNCs entailing at least one magnetic material component combined with semiconductors and/or metals, which hold potential for generating enhanced or unconventional magnetic behavior, while offering diversified or even new chemical-physical properties and functional capabilities. The available toolkit of synthetic strategies, all based on the manipulation of seeded-growth techniques, will be described, revisited, and critically interpreted within the framework of the currently understood mechanisms of colloidal heteroepitaxy.

Keywords: colloidal nanocrystals, heterostructures, epitaxy, seeded growth, topological control, surface energy, strain, magnetism 


\section{INTRODUCTION}

The development of methodologies and tools for the controllable fabrication, manipulation, and in-depth analysis of artificial nanostructures encodes an essential transition pathway to realizing the potential of the unique size-dependent properties of low-dimensional solids. In the realm of nanomaterials, colloidal inorganic nanocrystals (NCs), solution free-standing crystalline nanoparticles entirely synthesized and processable in liquid media, have now raised to the rank of model systems for assessing the foundations of the physical-chemical laws of nanoscale matter owing to the precision and flexibility with which their crystal habit, shape, dimensions, and surface moieties can be tailored in the preparation stage (Burda et al., 2005; Cozzoli et al., 2006; Cozzoli, 2008; Baghbanzadeh et al., 2011). In addition, NCs are robust and versatile enough to be exploited as key active elements in artificial mesoscopic materials, innovative processes, and devices of great fundamental and practical significance for optoelectronics (Talapin et al., 2010; Vanmaekelbergh, 2011), catalysis (Chng et al., 2013; Vaneski et al., 2014; Xu et al., 2016), energy conversion (Carey et al., 2015; Xu et al., 2016) and storage (Frey et al., 2009; Niederberger and Pinna, 2009; Lee and Cho, 2011; Oszajca et al., 2014), sensing (Freeman and Willner, 2012; Palui et al., 2015), environmental remediation (Tong et al., 2012; Wilker et al., 2012; Rawalekar and Mokari, 2013), and biomedicine (Parak et al., 2003; Michalet et al., 2005; Palui et al., 2015).

Colloidal approaches, which enable governing the thermodynamics and kinetics of nucleation, growth, and crystallization of solids in liquid media (Jun et al., 2006; Kwon and Hyeon, 2008; Erdemir et al., 2009; Wang et al., 2015), have demonstrated to be powerful synthetic routes to precisely phase-engineered NCs for a huge materials library across diverse size-morphological regimes (Cozzoli et al., 2006; Jun et al., 2006; Park et al., 2007; Cozzoli, 2008; Baghbanzadeh et al., 2011; Kim et al., 2013; Yang et al., 2013; Lhuillier et al., 2015). Recently, to meet the raising demand for intelligent nanosystems capable to exhibit enhanced, diversified, or even completely unprecedented properties and capabilities for multitask applications, synthetic nanochemistry research has opened up new horizons in the design, creation, and mastering of increasingly complex NC-based assemblies and architectures. At the forefront of progress in this area are last-generation breeds of colloidal systems, broadly referred to as heterostructured nanocrystals (HNCs), which are sophisticate all-solid-state multicomponent nanoparticles, distinguished by a spatially controlled distribution of their composition and structure. HNCs incorporate a countable number of discrete nanometer-scale modules (with the largest dimension smaller than $\sim 100-150 \mathrm{~nm}$ ) made of chemically and/or structurally different materials, which are welded together via direct solid-state chemically bonded heterointerfaces to form individually distinguishable, solution freestanding multifunctional hybrid nanoplatforms. In general, HNCs may group inorganic (Cozzoli et al., 2006; Buonsanti et al., 2007; Jun et al., 2007; Casavola et al., 2008; Carbone and Cozzoli, 2010; Talapin et al., 2010; de Mello Donegà, 2011; Liu et al., 2011; Buck and Schaak, 2013; Sitt et al., 2013; Banin et al., 2014; Melinon et al., 2014; Purbia and Paria, 2015; Qi et al., 2015) and/or organic materials, such as polymers (Lattuada and Hatton, 2011; Liu et al.,
2011; Zhang et al., 2012; He et al., 2013; Kaewsaneha et al., 2013; Pang et al., 2014; Purbia and Paria, 2015) or some carbon allotropes (Peng et al., 2009; Liu et al., 2011; Purbia and Paria, 2015; Yan et al., 2015b). As far as the attached domains grow crystalline, the relevant heterojunctions can develop epitaxially, allowing the concerned lattices to hold precise, yet synthetically adjustable, crystallographic, and spatial relationships relative to one other (Carbone and Cozzoli, 2010; Shim and McDaniel, 2010). Owing to these exclusive interface structure characteristics that guarantee stable inter-domain connectivity, HNCs can be safely processed in liquid media and relocated to different environments after the synthesis while maintaining their native configuration intact. Furthermore, HNCs can be controllably engineered with extremely rich structural and topological diversity, thus exhibiting integrated functionalities as well as novel properties stemming from the efficient electronic communication established between joint material domains. All these prerogatives are prohibited both to traditional colloidal nanocomposites that are constructed by exploiting weak (electrostatic or van der Waals) interactions or bifunctional molecules serving as linking bridges between the involved building blocks (Kamat, 2007; Quarta et al., 2007; Bigall et al., 2012; Tian et al., 2015; Yin et al., 2015) and to their heterocluster analogues (albeit frequently based on much larger nano-/micro-particle units) that are derived by assembly mechanisms relying on weak inter-particle forces, external perturbations, and the guide of templating substrates and interfaces (Peng et al., 2009; Duguet et al., 2011; Gao and Fang, 2015; Vogel et al., 2015; Yan et al., 2015a,b; Yin et al., 2015).

The most easily controllable prototypes of HNCs delivered so far can be roughly classified into two main categories: (i) core@shell architectures, in which the component domains are arranged in concentric or eccentric onionlike topologies, where only the outer shell material, which protects the inner core, is exposed to the external environment (Cozzoli et al., 2006; Buonsanti et al., 2007; Jun et al., 2007; Casavola et al., 2008; Carbone and Cozzoli, 2010; de Mello Donegà, 2011; Liu et al., 2011; Zhang et al., 2012; Kaewsaneha et al., 2013; Sitt et al., 2013; Banin et al., 2014; Melinon et al., 2014; Purbia and Paria, 2015; Qi et al., 2015) [in the yolk/shell variant, a core- or shellconformal void space may also intervene in the interior (Casavola et al., 2008; Carbone and Cozzoli, 2010; Liu et al., 2011; Purbia and Paria, 2015)]; (ii) non-core@shell segregated heteroclusters, in which the constituent sections are asymmetrically arranged in space through small heterojunctions, such that a substantial fraction of the surface of each material module remains accessible (Cozzoli et al., 2006; Buonsanti et al., 2007; Jun et al., 2007; Casavola et al., 2008; Peng et al., 2009; Carbone and Cozzoli, 2010; de Mello Donegà, 2011; Lattuada and Hatton, 2011; Buck and Schaak, 2013; Kaewsaneha et al., 2013; Sitt et al., 2013; Banin et al., 2014; Melinon et al., 2014; Pang et al., 2014; Yan et al., 2015b). The latter cluster-type heterostructures, which encompass two-component Janus-type HNCs (Cozzoli et al., 2006; Casavola et al., 2008; Carbone and Cozzoli, 2010; Lattuada and Hatton, 2011; He et al., 2013; Kaewsaneha et al., 2013; Pang et al., 2014) to multicomponent hetero-oligomer HNCs (Cozzoli et al., 2006; Casavola et al., 2008; Peng et al., 2009; Carbone and Cozzoli, 2010; Buck and Schaak, 2013; Yan et al., 2015b), are 
especially significant in that they can be regarded as "nanocrystal molecules," inorganic analogues of organic molecules equipped with a programmable number of functional moieties. Thus, in a sense, rational design and programmable fabrication of asymmetric non-core@shell HNCs conceptually mimic the total-synthesis approach used by organic chemists to construct large molecules. The synthetic toolkit of colloidal nanochemistry is being now leveraged toward development of reaction equivalents of predictable solid-state nanoparticle manipulations, such as addition, coupling, and condensation reactions, for elaborating exceptionally intricate NC-based architectures, in which an increasingly high level of structural-architectural sophistication and compositional wealth expands the assortment of affordable NC-enabled properties and applications.

Unlike any other family of nanoheterostructures, asymmetric non-core@shell magnetic heterostructured nanocrystals (MHNCs) promise to unveil unexpectedly new, broad, and exciting scenarios in both fundamental science and future technology. As of today, the available repertory of MHNCs has greatly expanded to the point of covering a broad selection of all-inorganic heterostructures (Cozzoli et al., 2006; Buonsanti et al., 2007; Casavola et al., 2008; Wang et al., 2009c; Carbone and Cozzoli, 2010; Buck and Schaak, 2013) as well as of organic-inorganic architectures (Wang et al., 2008; Peng et al., 2009; Feyen et al., 2010; Lattuada and Hatton, 2011; He et al., 2013; Kaewsaneha et al., 2013; Pang et al., 2014; Gao and Fang, 2015; Yan et al., 2015b), which conglobate magnetic transitionmetals (Fe, Ni, Co, Mn, and alloys thereof) and corresponding oxides, on one side, and non-magnetic transition-metals ( $\mathrm{Au}, \mathrm{Ag}$, $\mathrm{Pt}, \mathrm{Pd})$, oxides $\left(\mathrm{SiO}_{2}, \mathrm{TiO}_{2}, \mathrm{ZnO}\right)$, polymeric, and carbonaceous materials, on the other side. MHNCs undoubtedly exemplify prototypes of advanced nanoscale entities, where integration and synergistic interplay of the prerogatives of the constituent modules and proper engineering of their three-dimensional arrangement and interactions can underpin enhanced and/or diversified, or entirely unprecedented functionalities and capabilities. Primarily, robust, liquid-phase processable MHNCs obviously combine non-homologous chemical, optical, dielectric, and/or magnetic properties across distinct, yet permanently interconnected material sections featuring nanoscale spatial separation (Wang et al., 2008; Buonsanti et al., 2010; Feyen et al., 2010; Lattuada and Hatton, 2011; Buck and Schaak, 2013; He et al., 2013; Kaewsaneha et al., 2013; Pang et al., 2014). Second, MHNCs represent appealing multifunctional nanoplatforms on which new processes and applications can be founded and governed. These encompass, for example, the feasibility to assemble nanoparticle-based "superstructures," to carry out cooperative chemical conversions over magnetically recoverable catalytic grounds, to perform remote manipulations for assembly or delivery purposes, to install an anisotropic surface distribution of functional molecules, and to elaborate multimodal techniques for biomedical diagnostics and therapeutics (Cozzoli et al., 2006; Buonsanti et al., 2007; Jun et al., 2007; Casavola et al., 2008; Wang et al., 2009c; Carbone and Cozzoli, 2010; Feyen et al., 2010; Talapin et al., 2010; de Mello Donegà, 2011; Lattuada and Hatton, 2011; Liu et al., 2011; He et al., 2013; Kaewsaneha et al., 2013; Sitt et al., 2013; Banin et al., 2014; Melinon et al., 2014; Pang et al., 2014; Purbia and Paria,
2015; Qi et al., 2015; Yan et al., 2015b). Additionally, the direct electronic communication that is established in MHNCs across their interconnected material domains may underlie exchangecoupling interactions between non-homologous moieties, leading to modulated, synergistically reinforced, or even entirely unprecedented properties and functionalities, otherwise unreachable to their isolated material components and their physical-mixture counterparts. For example, MHNCs based on optically active semiconductors and/or noble metals supporting localized surface plasmon resonances (LSPRs) may exhibit anomalous absorption/emission and/or conductivity behavior due to modifications in electronic structure, degree of carrier confinement, recombination, separation and relocation dynamics of photostimulated charges carriers, and/or LSPR-to-exciton coupling (de Mello Donegà, 2011; He et al., 2013; Sitt et al., 2013; Banin et al., 2014; Melinon et al., 2014). In the case of MHNCs embodying magnetic phases and plasmonic metals, abnormally modified or mutually switchable magnetic, optical, and magneto-optical responses may reflect the synergistic interplay of magnetism, magneto-optical activity, and LSPR oscillations through various exchange-coupling mechanisms (Cozzoli et al., 2006; Jun et al., 2007; Casavola et al., 2008; Carbone and Cozzoli, 2010; Armelles et al., 2013; Pineider et al., 2013; López-Ortega et al., 2015). The possibility of achieving favorable electronic-structure hybridization at the heterointerfaces and of programing chargecarrier destination pathways across interfacial potential barriers of tunable height and widths in HNCs holds great fundamental and practical implications for (photo)catalytic, electrocatalytic, and chemical-sensing applications (Wang et al., 2009c; Costi et al., 2010; Chng et al., 2013; He et al., 2013; Rawalekar and Mokari, 2013; Banin et al., 2014; Song, 2015; Liao et al., 2016). These prerogatives suggest that creation of effective bonding junctions among selected nanoscale domains in appropriately configured MHNCs may be deliberately exploited to engineer their chemical-physical behavior and active functionalities, overcoming intrinsic limitations of single-material NCs (Jun et al., 2007; Casavola et al., 2008; Carbone and Cozzoli, 2010; de Mello Donegà, 2011; Buck and Schaak, 2013; Banin et al., 2014; LópezOrtega et al., 2015).

The tailored total synthesis of colloidal HNCs (including MHNCs) with predetermined topologies requires a high level of creativity in architectural design and exquisite synthetic ingenuity. The most widely exploited and effective approach to allinorganic HNCs relies on the so-called "seeded growth," whereby preformed NC seeds are manipulated at relatively high temperatures in a variety of ways: they can be used as (i) primary nucleation substrates for accommodating secondary domains of different materials upon heterogeneous reaction of the respective molecular precursors; (ii) starting building blocks that may be forced to weld to one another (Cozzoli et al., 2006; Casavola et al., 2008; Carbone and Cozzoli, 2010; Buck and Schaak, 2013); (iii) sacrificial templates into which foreign domains of other materials can be selectively implanted by inducing solid-state chemical-structural transformations and topological rearrangements (Cozzoli et al., 2006; Carbone and Cozzoli, 2010; Moon et al., 2011; Nag et al., 2014; De Trizio and Manna, 2016). In contrast to colloidal assembly techniques (Duguet et al., 2011; Vogel et al., 
2015; Yan et al., 2015a), seeded-growth routes guarantee the creation of genuine chemical bonds between dissimilar materials and can even allow the formation of epitaxial heterointerfacial connections in crystalline heterostructures (Carbone and Cozzoli, 2010; Shim and McDaniel, 2010).

Despite the seeming technical straightforwardness of the seeded-growth strategy and its successful applicability, the synthesis of HNCs with deliberately programmable spatial arrangements poses major inherent challenges. In fact, the formation of multimaterial architectures occurs at a critical thermodynamic-kinetic crossover, whereby the delicate dynamics that governs the structural and geometric evolution of individual constituent modules can heavily interplay with complex heterogeneous deposition or transformative growth pathways, which are, in turn, strongly affected by facet-dependent reactivity, interfacial strain, solidstate atomic diffusivity, and/or amenability to lattice ion exchange and/or incorporation (Cozzoli et al., 2006; Erdemir et al., 2009; Carbone and Cozzoli, 2010; Shim and McDaniel, 2010; Moon et al., 2011; Nag et al., 2014; De Trizio and Manna, 2016). Despite the current limited availability of general mechanistic knowledge on pathways to heterostructuring and the marginal understanding of their dependence on specific chemical routes, in many cases the growth dynamics of HNCs may be monitored and successfully governed on the basis of accurate empirical analysis of the synthetic impact of systematically varied reaction conditions on HNC product quality features (Hodges et al., 2016).

This review will provide a focused glimpse into this fastgrowing research field by illustrating recent advances made in the wet-chemical development and characterization of the valuable class of all-inorganic MHNCs with non-centrosymmetric topologies, a broad family of multifunctional entirely crystalline nanoheterostructures in which distinct material modules are clustered into non-core@shell multidomain architectures via bonding heterointerfaces of limited extension. Emphasis will be on heterostructures composed of monocrystalline domains with individually well-defined lattice orientation and surface faceting, which can feature defined structural and topological patterns with intervening epitaxial heterojunctions as a result of regioselective sequential growth pathways. Phase-segregated MHNCs of this type, which entail at least one magnetic metal or oxide material component structurally, chemically, and electronically connected to one or more magnetic or non-magnetic semiconductors, metals and/or metal oxides, hold remarkable potential not only as multifunctional nanosystems for catalysis, drug delivery, and biomedical theranostics, but also as nanoplatforms over which unconventional magnetic behavior and actively switchable magnetic and optical functionalities can be generated and manipulated to underpin the broad application spectrum of optoelectronics and, in particular, to propel the emerging field of magnetoplasmonics. For a wide range of material associations, various synthetic strategies, all of which rely on the application of seeded-growth technique variants, will be outlined, described and interpreted within the framework of the currently understood colloidal heteroepitaxy mechanisms. The unique structural and functional prerogatives offered by such generations of complex nanomaterials will also be shortly highlighted.

\section{BASIC PRINCIPLES UNDERLYING THE COLLOIDAL SYNTHESIS OF NANOCRYSTAL HETEROSTRUCTURES}

\section{Synthesis of Single-Material NCs}

Colloidal NCs are generated upon reaction of molecular precursors in a liquid medium that usually contains selected coordinating solvents and stabilizing agents (e.g., polyelectrolytes, ligands, polymers, surfactants, or soft self-assembled nanotemplates, such as micelles). The synthesis is triggered at a suitable temperature, at which highly reactive "monomers," the smallest building units (atomic species or clusters, or molecular fragments) with which the target material lattice may be constructed, are released upon conversion of the reactants and, above a critical supersaturation threshold, condense into a solid phase, thus initiating the nucleation of nanoparticles and sustaining their subsequent growth and crystallization (Ruckenstein and Djikaev, 2005; Erdemir et al., 2009; Gebauer and Colfen, 2011; Li et al., 2014). The organic stabilizers introduced into the liquid environment play several key roles during $\mathrm{NC}$ evolution: they can (i) form complexes with the monomers, thus dictating their actual chemical potential in the solution; (ii) dynamically adsorb onto/desorb from the surface of the growing NCs, guaranteeing controllable incorporation of monomers and steady growth; (iii) act as size- and shaperegulating agents; (iv) render the NCs highly soluble both in the synthesis environment and in other solvents of appropriate polarity after post-synthesis extraction and manipulation (Cozzoli et al., 2006; Jun et al., 2006; Kwon and Hyeon, 2008; Talapin et al., 2010).

Judicious adjustment of the temperature, the type, and the relative concentrations of precursors and organic stabilizers impacts on numerous thermodynamic conditions and kinetic processes underlying NC formation, including solution supersaturation, reactant and monomer diffusivity, lattice crystallization, solidstate in-lattice ion diffusivity, relative polymorph stability, and crystallographic-direction-dependent lattice growth rate (Cozzoli et al., 2006; Jun et al., 2006; Talapin et al., 2010). Experimental and theoretical insights have indicated that, in order to produce monodisperse NCs, a discrete, burst-like nucleation event must ideally be combined with a diffusion-controlled growth, and these two reaction stages should be temporally separated (Park et al., 2007; Kwon and Hyeon, 2008; Wang et al., 2015). Within this framework, systematically tuned sizes and narrow size variances can be achieved by balancing the relative consumption of monomers between the nucleation and the growth stages across an appropriately programed time evolution of the monomer concentration. Depending on the particular material target, this dynamics may be realized by applying ad hoc reactant delivery techniques (e.g., a primary swift "hot-injection," combined with secondary slow additions of extra reactants), by manipulating the unique reactivity of the system (e.g., a "delayed" nucleation event followed by rapid autocatalytic growth), or deliberate promotion of digestive ripening (to promote growth of the larger, more stable nanoparticles at the cost of the spontaneous dissolution of the smallest, unstable ones) (de Mello Donegà et al., 2005; Park et al., 2007; Kwon and Hyeon, 2008; Wang et al., 2015). Importantly, 
the dynamic binding of organic stabilizers can significantly affect the relative stability of the surface facets enclosing the growing NCs, thereby driving their shaping into non-spherical habits (e.g., cubes, polyhedrons, rods, wires, and polypods). In particular, anisotropic lattice development and branching-out, most frequently observed for materials forming in reduced-symmetry crystal phases and/or featuring polytypism during their evolution, may be promoted within kinetically dominated growth regimes and/or under conditions facilitating growth symmetry breaking (e.g., in the presence of soft surfactant or polymer lamellar templates, of foreign particle catalysts, or when crystal-oriented attachment pathways are allowed, or growth is conducted in external electric or magnetic fields or under microwave irradiation) (Cozzoli et al., 2006; Jun et al., 2006; Talapin et al., 2010; Zhang et al., 2010; Baghbanzadeh et al., 2011; Bouet et al., 2013; Hu and Wang, 2013; Li et al., 2013; Yang et al., 2013; Hu et al., 2014; Wang et al., 2014; Lhuillier et al., 2015).

\section{Simplified Thermodynamics of Heterostructure Formation}

In a thermodynamic perspective, the construction of a HNC may be formally viewed as a sequence of reaction steps, each involving the addition of one secondary material domain to a preexisting (hetero)nanocrystal "substrate" and the concomitant formation of a corresponding bonding solid-state heterointerface. In general, the material modules that are grouped together are likely to be both chemically and structurally dissimilar (i.e., crystallize in different phases and/or with dissimilar lattice parameters). At a first approximation, the energy balance underlying colloidal heterodeposition can be considered to be analogous to that accounting for the epitaxial growth of thin-film heterostructures and strained quantum dots onto large-area oriented substrates, performed by vapor-phase techniques (e.g., molecular beam epitaxy, chemical vapor deposition, etc.). This is illustrated in Figure 1. The sign of the total Gibbs free surface energy change function, $\Delta G_{S}$, that accompanies the heterogeneous deposition of a secondary material (2) over a primary preexisting substrate of a different composition/structure (1) will essentially dictate the growth mode of the former (Markov, 2003; Carbone and Cozzoli, 2010; Li et al., 2014):

$$
\Delta G_{\mathrm{S}}=\gamma_{1}-\gamma_{2}+\gamma_{1,2}
$$

where $\gamma_{1}$ and $\gamma_{2}$ are the solid/solution interfacial energies (also termed surface energies, i.e., the Gibbs free energies of solids 1 and 2 in contact with the solution per unit surface area, respectively) of the bare surfaces (facets) of the substrate and of the secondary material through which the heterojunction will be attained, and $\gamma_{1,2}$ is the solid/solid interfacial energy (i.e., the Gibbs free energy of solid 1 in contact with solid 2, or, equally, the Gibbs free energy of solid 1 in contact with solid 2, per unit interface area, respectively) associated with the formed heterointerface(s). Assuming that both $\gamma_{1}$ and $\gamma_{2}$ (hence, the surface energies of all facets exposed to the liquid environment) are not noticeably affected by the heterodeposition process itself, the $\gamma_{1}$ and $\gamma_{2}$ terms can be expected to be mainly influenced by the binding or adsorption of solution species (e.g., surfactants, ligands, and reactive monomers),

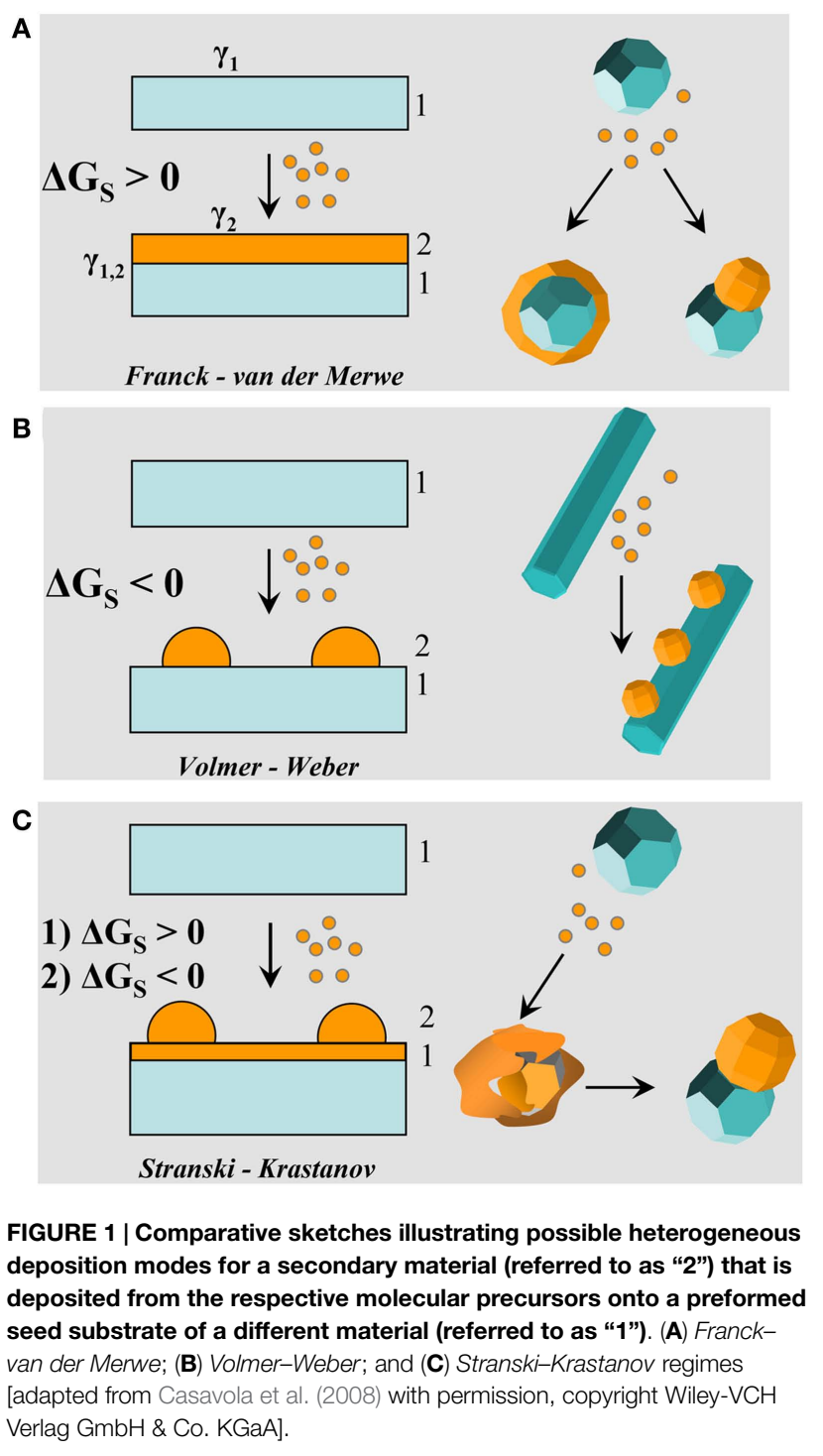

$\gamma_{1,2}$ will strictly depend on the bonding strength and degree of structural similarity of the involved lattices at the interfacial region.

If the secondary material exposes lower-energy surfaces (i.e., $\left.\gamma_{2}<\gamma_{1}\right)$ and/or can crystallographically match with the substrate to a satisfactory extent (hence, $\gamma_{1,2}$ is small), then its deposition will likely proceed layer-by-layer, resulting in a continuous and uniform coverage $\left(\Delta G_{S}>0\right.$ : Frank-van der Merwe mode in Figure 1A). As opposed, if the secondary material features higher-energy surfaces (i.e., $\gamma_{2}>\gamma_{1}$ ) and/or is significantly latticemismatched (hence, $\gamma_{1,2}$ is high), then it will tend to deposit as an array of island-to-droplet-like domains as a means of minimizing of the overall interfacial area (hence, the interfacial misfit strain) shared with the seed substrate underneath $\left(\Delta G_{S}<0\right.$ : Volmer-Weber mode in Figure 1B). The mean inter-domain distance would approximately scale with the extent of excess strain that has to be accommodated (Markov, 2003; Li et al., 2014). An evolutionary mixed-deposition regime could also be observed (Stranski-Krastanov mode in Figure 1C). In the early stages, the 
secondary material may grow according to a layer-by-layer mode $\left(\Delta G_{S}>0\right)$. Subsequently, as the deposited layer exceeds a threshold thickness and/or reaches a critical composition (for example, in those cases in which it chemically reacts with the substrate underneath, forming an alloy or solid solution), and/or an excess of thermal energy is provided (e.g., when reaction temperature is increased), subsequent growth continuation will proceed in the form of segregated domains protruding out of the initially deposited layer $\left(\Delta G_{S}<0\right)$ in response to an intensification of interfacial strain fields. Exceedingly high strain fields, combined with a strong cohesive energy of the secondary material, may also led to a complete dewetting of the initially deposited thin layer and its reshaping into discrete domains (Gentili et al., 2012; Thompson, 2012).

\section{Liquid-Phase Epitaxy via Seeded-Growth Routes}

The most widely exploited and facile "seeded growth" route to synthesize HNCs relies on liquid-phase heterogeneous deposition, which represents the solution-phase analogue of heteropitaxial growth performed by vapor-phase techniques. According to this approach, the liquid growth environment contains preformed NCs of a starting material, which serve as primary "seeds" for accommodating one or more secondary inorganic domains of a different material that is generated upon reaction of suitable molecular precursors. According to the classical nucleation theory, the energy barrier, $\Delta G_{\text {het }}^{*}$, that has to be surpassed for a foreign material "droplet" to nucleate heterogeneously onto a preexisting condensed phase (e.g., the seeds) is lower than the activation energy, $\Delta G_{\mathrm{hom}}^{*}$, required to trigger otherwise independent homogeneous nucleation of crystal embryos in the bulk liquid, according to Eq. 2 (Cozzoli et al., 2006; Jun et al., 2006; Park et al., 2007; Casavola et al., 2008; Kwon and Hyeon, 2008; Carbone and Cozzoli, 2010):

$$
\Delta G_{\mathrm{het}}^{*}=f(\theta) \Delta G_{\mathrm{hom}}^{*}
$$

where $f(\theta) \quad(0<f(\theta)<1)$ is the "wetting" function (Eq. 3) that depends on the contact angle $\theta$ formed between the substrate surface and droplet surface when the surface and interface tensions equilibrate at the three-boundary seed/nucleated "droplet"/solution-phase region (Eq. 4). (It is useful to recall that the surface tension is a vectorial quantity defined as the normal force per unit length which should be applied to create a line cut in the surface; therefore, in Eq. $4, \gamma_{1}$ and $\gamma_{2}$ rigorously represent the moduli of the surface tensions of the seed (1) and of the "droplet" (2), respectively, and $\gamma_{1,2}$ is the modulus of interfacial tension of the seed/"droplet" interface, which are dimensionally identical to the surface and interfacial energies appearing in Eq. 1.) In general, $\theta$ will depend on the nature of the materials, extension and structure of the exposed surfaces, geometry of the substrate seed and of the secondary domain deposited thereon, and on the free energy change accompanying the heterodeposition process (Eq. 1) (Markov, 2003; Carbone and Cozzoli, 2010; Jerison et al., 2011):

$$
\begin{aligned}
f(\theta) & =\frac{(2+\cos \theta)(1-\cos \theta)^{2}}{4} \\
\gamma_{1} & =\gamma_{2} \cos \theta+\gamma_{1,2}
\end{aligned}
$$

Note that the barrier for the growth of a heterogeneously nucleated domain, $\Delta G_{\text {growth }}^{*}$, is far smaller than both $\Delta G_{\text {hom }}^{*}$ and $\Delta G_{\text {het }}^{*}$ and corresponds to the limiting case of complete wetting $[f(\theta) \rightarrow 0$, for $\theta \rightarrow 0]$. In an equivalent way, heterogeneous nucleation can be understood as a process requiring a much lower chemical potential of solution monomers (proportional to their concentration) to be triggered, relative to homogenous nucleation:

$$
\Delta \mu_{\text {het }}<\Delta \mu_{\text {hom }}
$$

The deposition regimes predicable on the basis of Eq. 1 can be safely transcribed to the context of a seeded-growth synthesis, whereby the overall energy change accounting for the preference for a given topological configuration depends on how the surface $\left(\gamma_{1}, \gamma_{2}\right)$ and interfacial energy $\left(\gamma_{1,2}\right)$ terms competitively interplay with each other. For example, starting from a NC seed with well-defined facets, a foreign material can be deposited and evolve either into an ubiquitous shell (thus leading to a HNC with onionlike configuration) or into a discrete domain attached aside (thus leading to HNC with a heterodimer habit), if the conditions for a Frank-van der Merwe regime are either fulfilled for all facets exposed, or selectively for just one of them, respectively (Figure 1A). On the other hand, under circumstances favoring Volmer-Weber growth, one or more sufficiently extended facets of the original seeds may accommodate multiple domains of the foreign material, thus resulting in HNCs with patchy geometry (Figure 1B). In the intermediate case of a Stranski-Krastanov or dewetting regime, an initial metastable layered heteroarchitecture, where the secondary material has formed an ubiquitous thin coverage on a few or on all facets of the seed, can be observed to convert to a phase-segregated hetero-dimer/-oligomer topology as an efficient pathway toward lowering of excess interfacial strain (Figure 1C). Clearly, this evolution may be observed only when sufficient thermal energy is supplied to overcome the kinetic activation barrier for the restructuring under the specific synthesis conditions.

The formation of HNCs in seeded-growth syntheses may also proceed via pathways that do not necessarily involve heterogeneous nucleation/growth events. For example, under suitable conditions, in a reaction environment loaded with preformed lownuclearity HNC seeds (e.g., hetero-dimers) in the absence of extra molecular precursors, higher-order (hence, larger) heterostructures may be created by promotion of controlled aggregative growth, involving directional attachment of a discrete number of seeds into higher-nuclearity hetero-oligomer HNCs (Cozzoli et al., 2006; Casavola et al., 2008; Carbone and Cozzoli, 2010; Buck and Schaak, 2013). Alternatively, chemical, structural, and topological rearrangement of preexisting $\mathrm{NC}$ or HNC seeds into different HNCs may result from ion exchange, red-ox replacement reactions, and/or thermally driven phase segregation (Cozzoli et al., 2006; Carbone and Cozzoli, 2010; Moon et al., 2011; Nag et al., 2014; De Trizio and Manna, 2016). Thermodynamically, all these growth mechanisms and the predictable outcomes in terms of topologies preferentially or selectively achievable may still be safely described by evaluating the change in free surface energy accompanying the evolution of the starting seeds into the final HNC products (Eq. 1).

Experimental determination of the detailed formation mechanisms of colloidal heterostructures under given reaction 
conditions is a complex task that requires in situ and ex situ monitoring of the composition, structure, geometric, and topological features of HNCs captured at different growth stages (Hodges et al., 2016). Such study can be carried out by combining state-of-art scanning electron microscopy and transmission electron microscopy (TEM), high-angle annular dark-field imaging in scanning transmission electron microscopy mode (HAADF-STEM), phase-contrast high-resolution transmission electron microscopy (HRTEM), electron diffraction, TEM tomography, and elemental microanalysis techniques available in a TEM microscope (Midgley and Weyland, 2003; Seyring et al., 2011; Ringe, 2014; Ross, 2015; Carenco et al., 2016), with electron and X-ray scattering and diffraction approaches (Ringe, 2014; Ghigna and Spinolo, 2015; Giannini et al., 2016; Li et al., 2016). Indirect, yet complementary, information can be gathered by monitoring the time evolution of the pertinent optical and magnetic properties.

Finally, it is important to stress that the creation of nanoscale heterointerfaces in a colloidal solution can benefit from the binding of organic stabilizers or other solution species, which can significantly influence the surface energy terms (i.e., $\gamma_{1}$ and $\gamma_{2}$ ), to the point that even large $\gamma_{1,2}$ may be significantly compensated for. This opportunity lays the basis for the high synthetic flexibility of colloidal epitaxy routes, which can thus be expected to open an avenue to "exotic" HNCs made of rather structurally dissimilar materials that would otherwise be difficult to forge into the form of epitaxial nanoheterostructures.

\section{ASYMMETRIC HETEROSTRUCTURES}

The configuration in which MHNCs (and HNCs in general) have most frequently been engineered is the so-called core@shell topology. In such systems an inner NC "core" is evenly enwrapped within a "shell" made of one or more layers of other materials, which ultimately governs or mediates MHNC interactions with the external environment. Semiconductors, metals, and oxides arranged in centrosymmetric onionlike or eccentric core@shell configurations share large connecting heterointerfaces, across which direct electronic communication and hybridization may lead not only to chemical-physical properties distinct from those inherent to the individual components (e.g., increased photoluminescence, emission over spectral ranges prohibited to the individual material components alone, enhanced or modified LSPR absorption, increased magnetic anisotropy, enhanced ionintercalation capabilities, and unexpected catalytic activity), but also to exchange interactions of non-homologous properties (e.g., exciton-LSPR coupling, exchange coupling between different magnetic phases), depending on the specific material association (Casavola et al., 2008; Carbone and Cozzoli, 2010; Talapin et al., 2010; Ghosh Chaudhuri and Paria, 2011; Lee and Cho, 2011; Liu et al., 2011; Su et al., 2011; Chatterjee et al., 2014; Melinon et al., 2014; Oszajca et al., 2014; Purbia and Paria, 2015).

Magnetic heterostructured nanocrystals with coherently grown epitaxial interfaces may be attained when the core and shell materials are characterized by similar crystal phase and closely matching lattice parameters, two structural prerequisites that guarantee misfit strain to be kept acceptably low and prevent the generation of defects as long as the coating thickness is sufficiently small. However, various circumstances may occur under which the requirements of lattice compatibility can be fairly less restrictive (Cozzoli et al., 2006; Carbone and Cozzoli, 2010; Melinon et al., 2014). Pathways enabling plastic strain relaxation may be available when the deposition proceeds non-epitaxially, for example, when the shell grows polycrystalline or amorphous. Misfit strain constraints could easily be circumvented when shell growth is accomplished under kinetically driven conditions, yet at the cost of incorporation of a large density of crystal defects (e.g., dislocations, stacking faults) at the interfacial regions and/or within the shell. In such cases, the core/shell interface may ultimately entail a number of small-area coherent heterojunctions at which dissimilar crystallographic relationships may locally hold between the joint lattices. Alternatively, the ligand environment may allow an otherwise excedingly high interfacial energy to be efficiently offset by a proportional decrease in surface energy associated with the outermost exposed shell surface (Casavola et al., 2008; Carbone and Cozzoli, 2010; Melinon et al., 2014; López-Ortega et al., 2015).

Over the past decade, however, a high degree of heterostructure diversity has been mastered by colloidal seeded-growth routes, which have allowed access to MHNCs with non-core@shell topologies. The available broad library comprises prototypes of "nanocrystal molecules" featuring a spatially asymmetric distribution of their composition and crystal structure. These are heterodimer, hetero-oligomer, and anisotropic multidomain MHNCs that integrate distinct size- and shape-controlled sections of dissimilar materials interconnected through one or multiple solid-state heterojunction, without any organic molecular bridges. Distinct from their onionlike counterparts, the modular, most frequently asymmetric configuration of these nanoheterostructures with inherent multifunctionality, is conceptually reminiscent of the architecture of complex polyfunctional molecules, whereby organic moieties are correspondingly replaced by domains of different inorganic materials (Duguet et al., 2011; Buck and Schaak, 2013). These MHNCs not only group the distinctive properties of their constituents, allowing electronic interactions among them, but also offer diversified sets of surface platforms onto which a topologically controlled distribution of functional moieties may eventually be anchored (Cozzoli et al., 2006; Jun et al., 2007; Casavola et al., 2008; Carbone and Cozzoli, 2010; de Mello Donegà, 2011; Buck and Schaak, 2013).

In seeded-growth synthesis, various growth conditions can make MHNC deviate from a core@shell development regime. Under thermodynamically controlled conditions, the topology will be dictated by the ultimate surface energy balance accompanying the deposition event or heterostructuring process (Eq. 1). For example, materials that do not form alloys and/or are strongly lattice-uncorrelated can evolve into heterodimer/-oligomer-type heterostructures as a pathway toward minimizing the overall interfacial strain at a proportionally smaller cost of increased surface energy (associated to the multiple material surfaces exposed). Other circumstances favoring the formation of non-core@shell MHNCs may related to the introduction of seeds characterized by an apparent site-preferential accessibility or chemical reactivity that could arise from the variable degrees of lattice matching 
achievable at the exposed facets, or from kinetically driven deposition conditions at selected locations (Cozzoli et al., 2006; Jun et al., 2007; Casavola et al., 2008; Carbone and Cozzoli, 2010; de Mello Donegà, 2011; Buck and Schaak, 2013). Equally, small bonding junctions may be induced to form among preexisting MHNCs as a means of alleviating the high surface energy that would otherwise characterize their physical mixtures in solution phase, for example, in the case of ineffective ligand stabilization of selected domain surfaces (Cozzoli et al., 2006; Carbone and Cozzoli, 2010).

\section{Hetero-dimers and Hetero-Oligomers Grouping Nearly Isotropic-Shaped Material Domains}

A variety of hetero-dimer and hetero-oligomer MHNCs that are composed of two or more isotropically shaped (e.g., spherical, cubic, and polyhedral) modules can readily be prepared, within the framework of seeded-growth based techniques, by exploitation of different mechanisms of heterostructuring: (a) direct heterogeneous nucleation; (b) non-epitaxial shell deposition followed by thermally driven crystallization and dewetting; (c) reactions at liquid/liquid interfaces; (d) self-regulated homogeneous-heterogeneous nucleation and growth; and (e) induced attachment of preformed MHNCs. Representative examples that illustrate the level of architectural precision and sophistication achievable are collected in Figures 2-6 and described in the following paragraphs.

\section{Heterogeneous Nucleation \\ Direct Heterogeneous Nucleation}

Direct heterogeneous nucleation (Figure 2A) is among the most frequently exploited pathways for constructing MHNCs in the form of binary and ternary assemblies composed of diverse associations of magnetic, metal, and materials. Hetero-dimer and higher-order hetero-oligomer MHNCs have been envisioned as key elements on which new technological solutions may be envisaged, especially in fields where multifunctionality and multitasking capabilities are intrinsically needed (e.g., sensing, imaging, drug delivery, and therapy in biomedicine). For example, it has been proposed that distinct material modules of a HNC can be used as anchoring platforms for the site-specific attachment of selected biomolecules or ligands (Jun et al., 2007; Gao et al., 2009; Bigall et al., 2012; Lim and Majetich, 2013). In addition, while a metal or semiconductor domain can enable optical detection (e.g., via excitonic or LSPR absorption, or photoluminescence), a magnetic module can be utilized for complementary purposes, such as for magnetic resonance imaging (MRI), optical imaging, and magnetic separation (Choi et al., 2006, 2008; Jun et al., 2007; Jiang et al., 2008; Xu et al., 2008; Gao et al., 2009; Schladt et al., 2010; Bigall et al., 2012; Lim and Majetich, 2013). The existence of bonding heterointerfaces through which dissimilar materials can electronically communicate has clearly been recognized to impact on the magnetic (Xu et al., 2008; Lee et al., 2010a; Umut et al., 2012; Pineider et al., 2013; Kim and Song, 2014; Schick et al., 2014; LópezOrtega et al., 2015; Velasco et al., 2015), optical (Levin et al.,
2009; Korobchevskaya et al., 2011; Comin et al., 2012), transport (Lee et al., 2010a), magneto-optical (Li et al., 2005; Armelles et al., 2013), (electro)catalytic (Yin et al., 2008; Wang et al., 2009a, 2010; Wu et al., 2009; Lee et al., 2010b; George et al., 2011a, 2013; Jang et al., 2011b; Lin and Doong, 2011; Chen et al., 2012; Sun et al., 2012), and energy-storing properties of appropriately engineered MHNCs (Liu et al., 2015). Examples of MHNCs derived from heterogeneous deposition pathways are collected in Figures 2B-Q.

Thermal decomposition of metallorganic precursors in the presence of preformed noble metal, $\mathrm{Fe}_{3} \mathrm{O}_{4}, \mathrm{FePt}$, or $\mathrm{UO}_{2}$ seeds in non-coordinating solvents, such as octadecene (ODE) or phenyl ether containing oleic acid (OLAC), oleyl amine (OLAM), and/or tri- $n$-octyl phosphine (TOP) surfactants at $200-300^{\circ} \mathrm{C}$ has enabled access to hetero-dimer HNCs made of two nearly spherical and/or cubic-shaped domains epitaxially interconnected, such as of $\mathrm{Me}-\mathrm{Fe}_{3} \mathrm{O}_{4}(\mathrm{Me}=\mathrm{Au}, \mathrm{AuAg}, \mathrm{PtPd}, \mathrm{AuPd}, \mathrm{AuPt}, \mathrm{Pt}, \mathrm{Pd}, \mathrm{Ni}$, $\mathrm{Cu}$ ) (Yu et al., 2005; Shi et al., 2006b; Choi et al., 2008; Wei et al., 2008; Wang et al., 2009a, 2010; George et al., 2011a, 2013; Jang et al., 2011a; Lin and Doong, 2011; Nakhjavan et al., 2011; Zhai et al., 2011; Leung et al., 2012; Sun et al., 2012; Kim and Song, 2014; Victor et al., 2015), $\mathrm{Fe}_{3} \mathrm{O}_{4}-\mathrm{MnO}$ (Lee et al., 2012), $\mathrm{Au}-\mathrm{MnO}$ (Choi et al., 2008; Schladt et al., 2010), $\mathrm{Fe}_{3} \mathrm{O}_{4}-\mathrm{CdSe}$ (Tao et al., 2009), $\mathrm{UO}_{2}-\mathrm{In}_{2} \mathrm{O}_{3}$ (Wu et al., 2011a), and FePt-YZ (YZ $=\mathrm{CoFe}_{2} \mathrm{O}_{4}, \mathrm{Co}$, $\left.\mathrm{Ni}, \mathrm{Fe}, \mathrm{CdS}, \mathrm{ZnS}, \mathrm{PbS}, \mathrm{PbSe}, \mathrm{CdSe}, \mathrm{In}_{2} \mathrm{O}_{3}, \mathrm{Fe}_{3} \mathrm{O}_{4}, \mathrm{MnO}\right)(\mathrm{He}$ et al., 2009; Lee et al., 2010a; Wu et al., 2011a; Schladt et al., 2012; Liu et al., 2014; Yang et al., 2015), respectively. Depending on the geometric features of the two-component domains, these heterostructures exhibit various morphological profiles, spanning from peanut-, dumbbell-, brick- to flower-like (Figures 2B-D). In most cases, the non-negligible difference in lattice parameters has been considered to be the main driving force that promotes extensive segregation of the concerned materials into discrete domains, oriented relative to one another so as to guarantee coherent small-area heterointerfaces and minimal misfit strain. Among these cases, the formation mechanism of $\mathrm{Au}-\mathrm{Fe}_{3} \mathrm{O}_{4}$ hetero-dimers was investigated in detail (Yu et al., 2005; Wei et al., 2008). The solvent was found to play a fundamental role in regulating the density of $\mathrm{Fe}_{3} \mathrm{O}_{4}$ nucleation sites on the Au seeds. The dumbbelllike configuration yielded by reactions performed in non-polar media was explained by invoking induction of polarization charge at those regions of the $\mathrm{Au}$ seeds where $\mathrm{Fe}_{3} \mathrm{O}_{4}$ had initially been deposited. Such polarization resulted in corresponding electron density depletion at other locations, where further nucleation events could in fact be inhibited. By contrast, in syntheses carried out in a more polar electron-donor solvent, any electron deficiency generated over the $\mathrm{Au}$ surface could be compensated for and leveled off by the medium molecules, which contributed to render the seed a more suitable ground either for the installation of multiple $\mathrm{Fe}_{3} \mathrm{O}_{4}$ "petals" or for the accommodation of a uniform $\mathrm{Fe}_{3} \mathrm{O}_{4}$ coverage (Yu et al., 2005; Wei et al., 2008; Wang et al., 2010).

In additional mechanistic investigations on other metal/metal oxide and metal/semiconductor systems, the transition from dumbbell- to flower-like geometry was controllably achieved by increasing the temperature and/or relative precursor to seed proportions (Shi et al., 2006b; Choi et al., 2008; Jiang et al., 2008; 


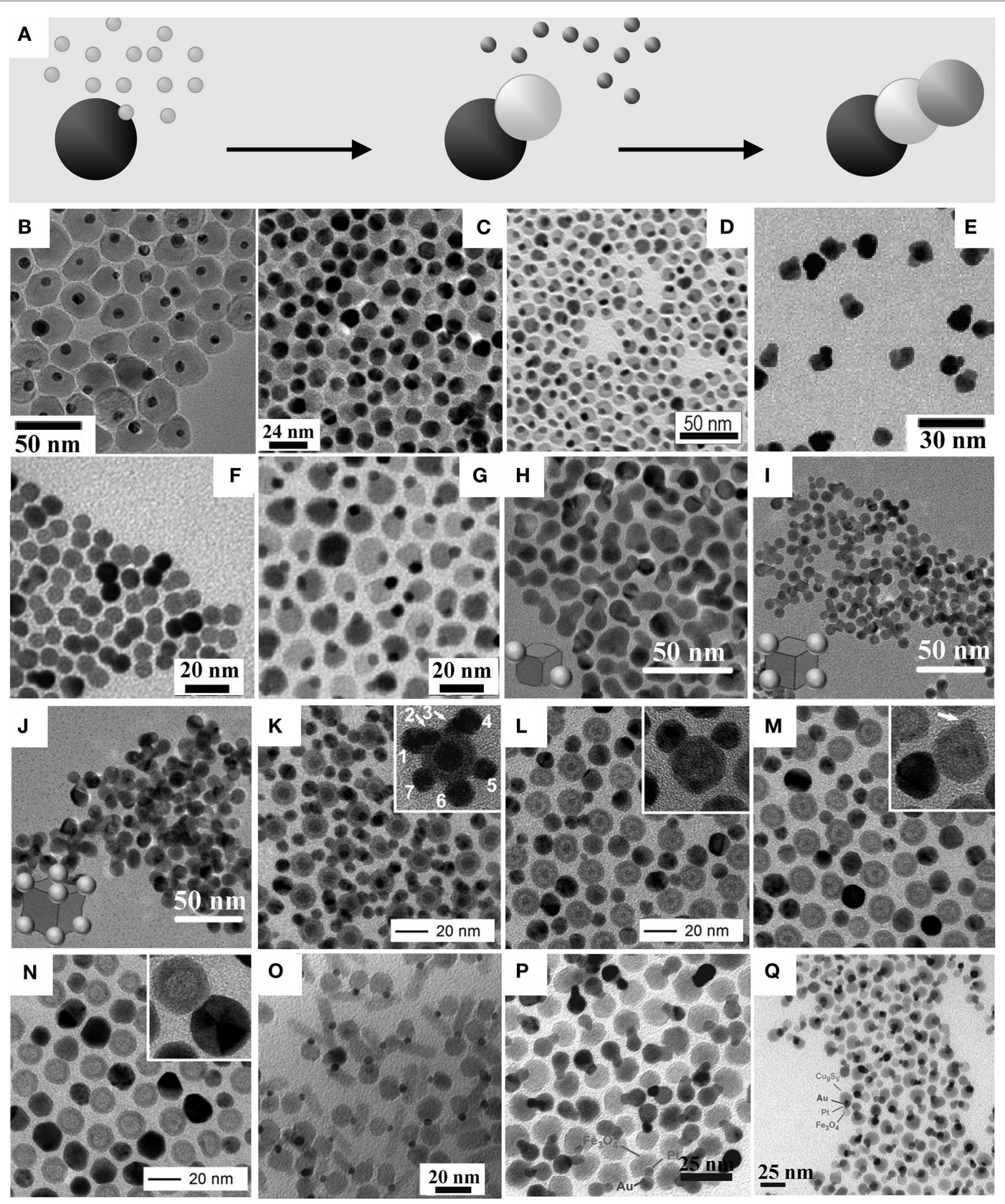

FIGURE 2 | Examples of hetero-dimers and hetero-trimer magnetic heterostructured nanocrystals (MHNCs) synthesized by direct epitaxial heterogeneous nucleation onto preformed seeds. (A) sketch of the mechanism [adapted from Casavola et al. (2008) with permission, copyright Wiley-VCH Verlag GmbH \& Co. KGaA]. (B-Q) Low-magnification transmission electron microscopy images of: (B) peanut-shaped Au-MnO MHNCs [adapted from Choi et al. (2008) with permission, copyright American Chemical Society]; (C) dumbbell-like Au-Fe $\mathrm{O}_{4} \mathrm{MHNCs}$ [reproduced from Yu et al. (2005) with permission, copyright American Chemical Society]; (D) peanut-shaped FePt- $\mathrm{In}_{2} \mathrm{O}_{3}$ hetero-dimer MHNCs with cubic-shaped FePt domains [reproduced from Wu et al. (2011a) with permission, copyright American Chemical Society]; (E) FePt-Au hetero-dimer MHNCs made of cubic-shaped FePt and spherical-shaped Au domains [reproduced from Choi et al. (2006) with permission, copyright American Chemical Society]; (F) symmetric CoPt ${ }_{3}-\mathrm{Au}$ hetero-dimer MHNCs synthesized according to Pellegrino et al. (2006); (G) peanut-shaped Pt- $\mathrm{Fe}_{3} \mathrm{O}_{4}$ MHNCs [adapted from Wang et al. (2010) with permission, copyright American Chemical Society]; (H-J) trimeric and oligomeric FePt-Au heterostructured nanocrystals grown starting from cubic FePt seeds [reproduced from Zhu et al. (2013) with permission from The Royal Society of Chemistry]; (K-N) evolution from Fe@Fe $\mathrm{O}_{y}-\mathrm{Ag}$ hetero-oligormer MHNCs to lower-order hollow-Fe@ $\mathrm{Fe}_{x} \mathrm{O}_{y}-\mathrm{Ag}$ hetero-oligormers to hollow-Fe $\mathrm{O}_{y}-\mathrm{Ag}_{\mathrm{g}}$ hetero-dimers via ripening and Kirkendall diffusion [reproduced with permission from Peng et al. (2011), copyright Wiley-VCH Verlag GmbH \& $\mathrm{Co}$. $\mathrm{KGaA}$ ]; (0) Au-Fe $3 \mathrm{O}_{4}-\mathrm{PbS}$ hetero-trimer MHNCs obtained by nucleating a rod-shaped PbS section on the Au domain of $\mathrm{Au}-\mathrm{Fe}_{3} \mathrm{O}_{4}$ hetero-dimer seeds [reproduced from Shi et al. (2006b) with permission, copyright American Chemical Society]; $(\mathbf{P}, \mathbf{Q}) \mathrm{Ag}-\mathrm{Pt}-\mathrm{Fe}_{3} \mathrm{O}_{4}$ and $\mathrm{Cu}_{9} \mathrm{~S}_{5}-\mathrm{Pt}_{-}-\mathrm{Fe}_{3} \mathrm{O}_{4}$ hetero-trimer $\mathrm{MHNCs}$ obtained by chemoselective nucleation of Au or $\mathrm{Cu}_{9} \mathrm{~S}_{5}$ on the $\mathrm{Pt}$ domain of $\mathrm{Pt}-\mathrm{Fe}_{3} \mathrm{O}_{4}$ hetero-dimer seeds [reprinted from Buck et al. (2012) by permission from Macmillan Publishers Ltd, copyright 2012]. 

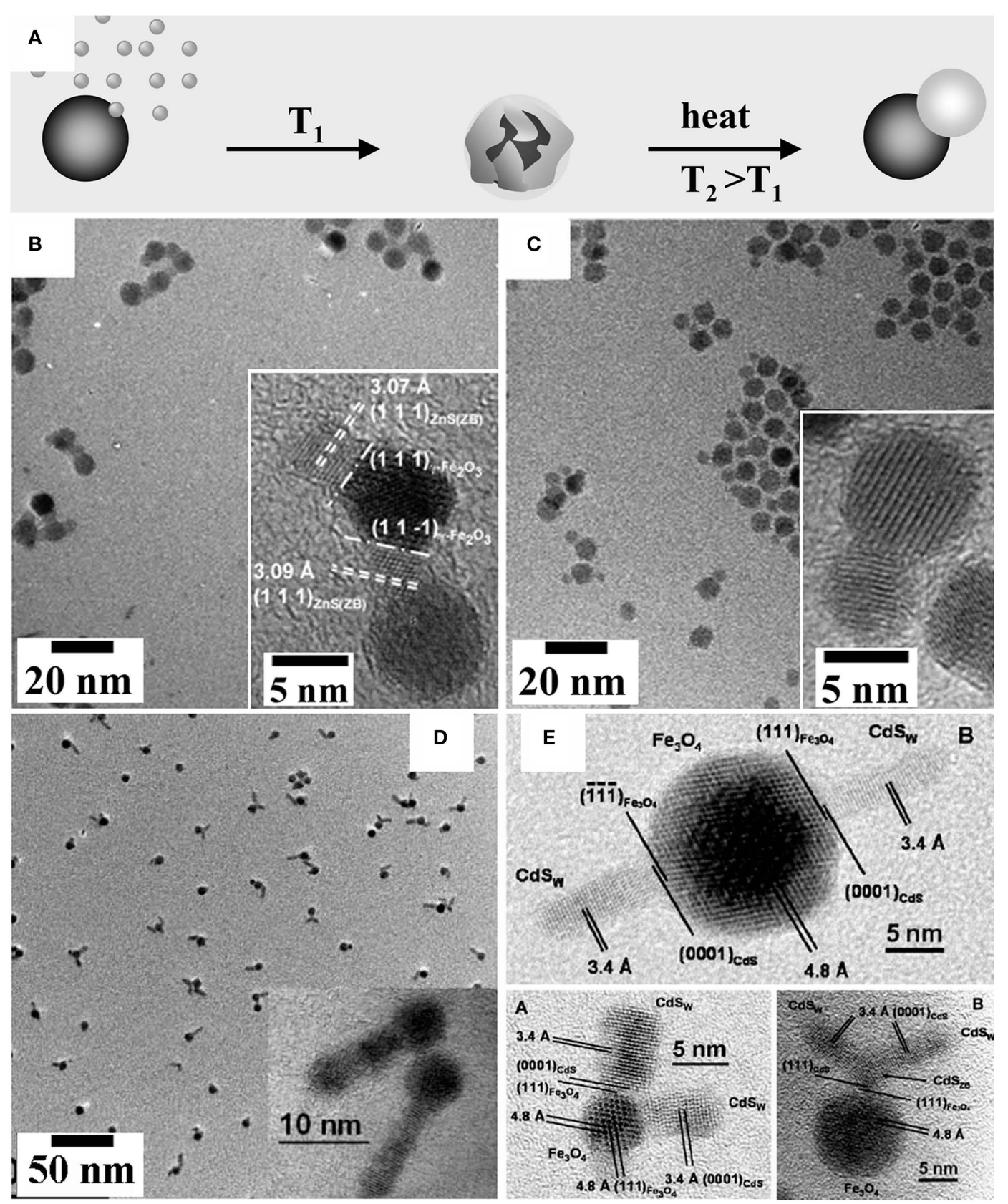

FIGURE 3 | Examples of heterocluster magnetic heterostructured nanocrystals (MHNCs) synthesized by post-deposition crystallization, coalescence, and dewetting. (A) Sketch of the mechanism [adapted from Casavola et al. (2008) with permission, copyright Wiley-VCH Verlag GmbH \& Co. KGaA]. (B-E) Low-magnification transmission electron microscopy galleries and selected high-resolution transmission electron microscopy images of: (B,C) $\mathrm{Fe}_{3} \mathrm{O}_{4}-\mathrm{ZnS}$ hetero-dimers and hetero-oligomer MHNCs [adapted from Kwon and Shim (2005) with permission, copyright American Chemical Society]; (D,E) Fe $\mathrm{O}_{4}-\mathrm{CdS}$ anisotropic MHNCs with one or multiple rod-shaped CdS sections departing from a single spherical $\mathrm{Fe}_{3} \mathrm{O}_{4}$ seed [reproduced from McDaniel and Shim (2009) with permission, copyright American Chemical Society].

Wei et al., 2008; He et al., 2009; Schladt et al., 2010; Zhai et al., 2011). In some cases, the nature of ligands that were originally bound to the surface of the starting seeds or were added during the seeding stage was found to be critical to driving the preference for a hetero-dimer topology over a core@shell one, which pointed to the influence of kinetic processes on topology selection (Yu et al., 2005; Choi et al., 2008; Jiang et al., 2008; Wei et al., 2008; He et al., 2009; Lee et al., 2010a; Schladt et al., 2010; Nakhjavan et al., 2011; Zhai et al., 2011).

In an effort to rationalize the formation of peanut-shaped FePt- $\mathrm{In}_{2} \mathrm{O}_{3}$ hetero-dimer MHNCs (Wu et al., 2011a), the crystallographic relationships holding between two materials were first investigated on the basis of the coincidence site lattice theory (CSLT) (Randle, 1997; Kwon and Shim, 2005; Kwon et al., 2006; 


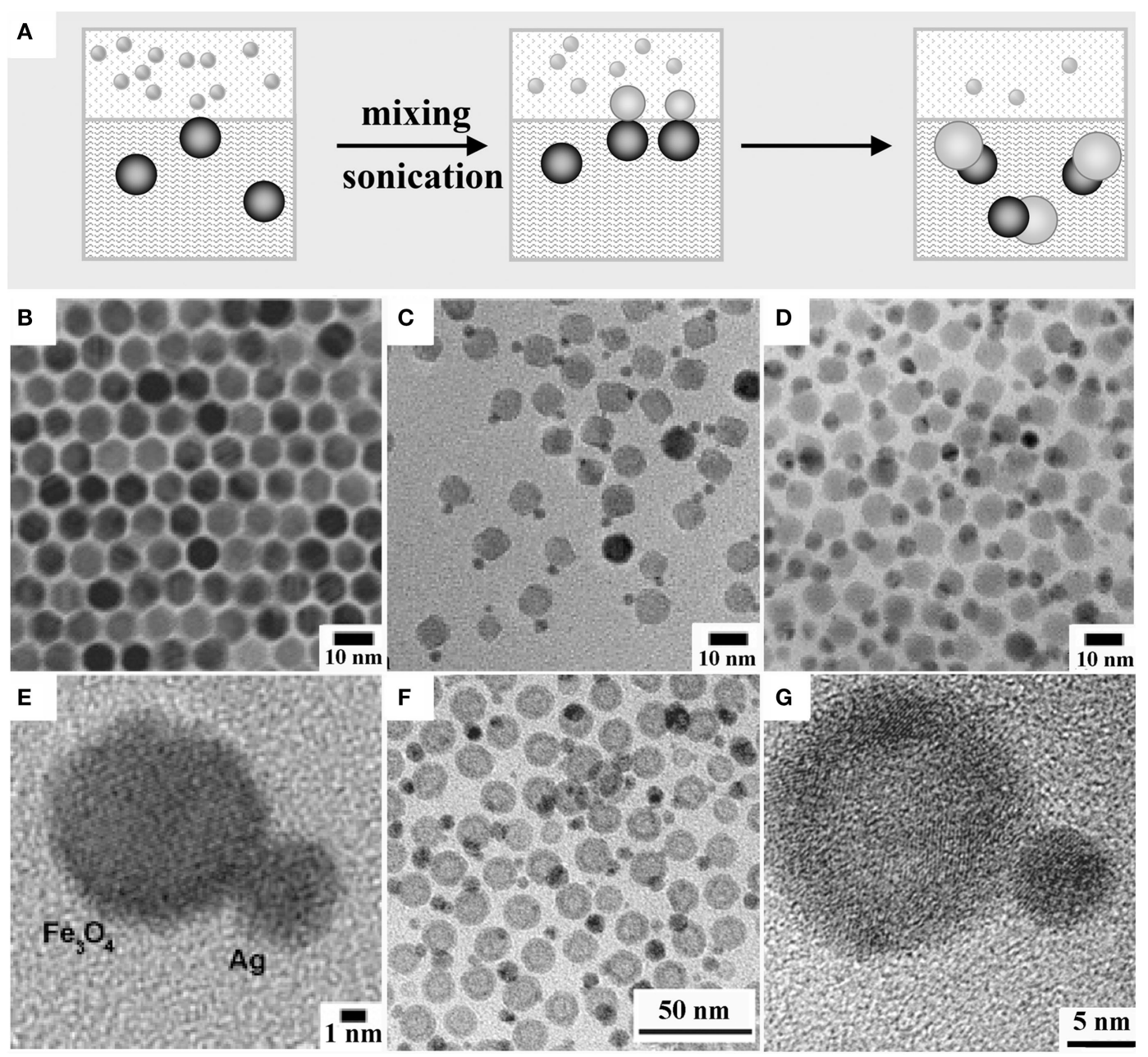

FIGURE 4 | Examples of hetero-dimer magnetic heterostructured nanocrystals (MHNCs) synthesized upon heterogeneous nucleation liquid/liquid interfaces. (A) Sketch of the mechanisms [adapted from Casavola et al. (2008) with permission, copyright Wiley-VCH Verlag GmbH \& Co. KGaA]. (A-G) Variable-magnification transmission electron microscopy images of: (B) starting $\mathrm{Fe}_{3} \mathrm{O}_{4}$ seeds; (C-E) $\mathrm{Fe}_{3} \mathrm{O}_{4}$-Ag hetero-dimers thereof with different Ag domain size [reproduced from Gu et al. (2005) with permission, copyright American Chemical Society]; (F,G) $\mathrm{Fe}_{3} \mathrm{O}_{4}-\mathrm{Ag}$ hetero-dimers made of one solid $\mathrm{Ag}$ domain and one hollow- $\mathrm{Fe}_{3} \mathrm{O}_{4}$ domain [adapted from Pan et al. (2010) with permission, copyright American Chemical Society].

McDaniel and Shim, 2009). In the CSLT approach, starting from the assessment of the relative domain orientations in a MHNC upon analysis of the relevant HRTEM data, the degree of matching between points of the concerned lattices and the frequency at which this correspondence occurs along the relevant heterojunction planes can be studied. Several couples of facets of the FePt and $\mathrm{In}_{2} \mathrm{O}_{3}$ lattices were identified to be potentially involved in the formation of satisfactorily lattice-matched heterointerfaces. An empirical law, referred to as a "bonding energy criterion," was then proposed to explain the preference for the few particular relative FePt to $\mathrm{In}_{2} \mathrm{O}_{3}$ lattice orientations that were observed experimentally in the hetero-dimers: instead of assuming that $\operatorname{In}_{2} \mathrm{O}_{3}$ overgrowth took place on the facets of the FePt seeds, at which lattice mismatch could be minimized, the epitaxial deposition of $\operatorname{In}_{2} \mathrm{O}_{3}$ was considered to most be favored on the FePt crystal facets for which the first atomic monolayer of the deposited $\mathrm{In}_{2} \mathrm{O}_{3}$ had the strongest chemical affinity (Wu et al., 2011a).
By a reverse reaction scheme, hetero-dimers made of $\mathrm{FePt}-\mathrm{Au}$ (Mokari et al., 2005; Choi et al., 2006; Shi et al., 2006b; Selvan et al., 2007; Wark et al., 2008; He et al., 2009; Franchini et al., 2010; Zeng et al., 2010; Mao et al., 2011; Krylova et al., 2012; Zhu et al., 2013), $\mathrm{CoPt}_{3}-\mathrm{Au}$ (Pellegrino et al., 2006; PazosPerez et al., 2007; Krylova et al., 2012), and $\mathrm{Fe}_{3} \mathrm{O}_{4}-\mathrm{Ag}$ (Zhang et al., 2006; Jiang et al., 2008; Yang and Ying, 2009; Huang et al., 2011; Peng et al., 2011; Mao et al., 2013) were generated upon reduction of $\mathrm{Au}(\mathrm{I})-, \mathrm{Au}(\mathrm{III})-$, or $\mathrm{Ag}(\mathrm{I})$-ligand complexes onto $\mathrm{FePt}, \mathrm{CoPt}_{3}$, and $\mathrm{Fe}_{3} \mathrm{O}_{4}$, seeds, respectively, with mild reducing agents (alkyl amines, alkyl diols, $\mathrm{Ar} / \mathrm{H}_{2}$ atmosphere) at moderate temperatures $\left(<120^{\circ} \mathrm{C}\right)$ (Figures 2E-G). With the introduction of an extra reaction step, $\gamma-\mathrm{Fe}_{2} \mathrm{O}_{3}-\mathrm{Cu}_{2} \mathrm{O}$ and $\mathrm{Au}-\mathrm{Fe}_{3} \mathrm{O}_{4}$ heterodimer MHNCs were produced from parent $\gamma-\mathrm{Fe}_{2} \mathrm{O}_{3}-\mathrm{Cu}$ (Mirtchev et al., 2014) and $\mathrm{Au}-\mathrm{Fe}$ (Jiang et al., 2016) hetero-dimers upon post-synthesis air oxidation of their $\mathrm{Cu}$ and Fe sections, respectively. 


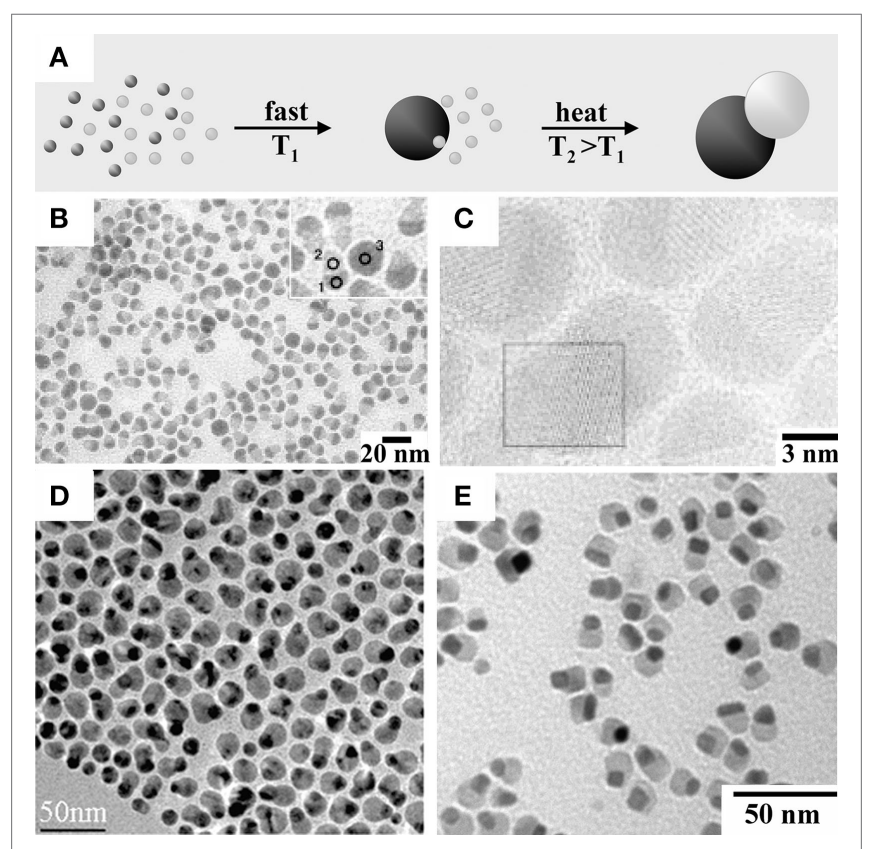

FIGURE 5 | Examples of magnetic heterostructured nanocrystals (MHNCs) synthesized by self-controlled nucleation-growth mechanisms. (A) Sketch of the mechanism [adapted from Casavola et al. (2008) with permission, copyright Wiley-VCH Verlag GmbH \& Co. KGaA]. (B-E) Low-magnification transmission electron microscopy overviews and selected high-resolution transmission electron microscopy images of: (B,C) acorn-shaped $\mathrm{Co}_{9} \mathrm{~S}_{8}-\mathrm{PdS}_{x}$ hetero-dimers [reproduced from Teranishi et al. (2004) with permission, copyright American Chemical Society]; (D) Au-Ni hetero-dimers [reproduced from Wang and Li (2010) with permission, copyright American Chemical Society]; (E) peanut-shaped $\mathrm{FePt}-\mathrm{Fe}_{3} \mathrm{O}_{4}$ hetero-dimers [reproduced from Figuerola et al. (2008) with permission, copyright American Chemical Society].

The properties of these magnetic/metal/semiconductor MHNCs have been found to clearly diverge from those of the individual components alone. For example, the luminescence of semiconductor modules is severely quenched due to the metal contact promoting electron transfer, hence, decreasing the probability of radiative electron-hole recombination (de Mello Donegà, 2011; Sitt et al., 2013; Banin et al., 2014). The LSPR features of coinage-metal sections are largely modified, depending on the dielectric characteristics of their proximal neighbors (Mokari et al., 2005; Shi et al., 2006b; Yang et al., 2006a,b, 2009; Yang and Ying, 2009; Pazos-Perez et al., 2007; Selvan et al., 2007; Wark et al., 2008; He et al., 2009; Franchini et al., 2010; Zeng et al., 2010). In addition, the relevant magnetic parameters of magnetic domains are often found to unpredictably deviate from those of the otherwise isolated components (Choi et al., 2006; Huang et al., 2011; Mao et al., 2011; Wu et al., 2011b; Leung et al., 2012; Zhu et al., 2013), indirectly revealing an influence of interfacial electron communication on the electronic and magnetic behavior (Pellegrino et al., 2006; Shi et al., 2006b; Zhang et al., 2006; Pazos-Perez et al., 2007; Jiang et al., 2008; Yang and Ying, 2009; Yang et al., 2009).

Further mechanistic knowledge on MHNC formation has been grasped upon analysis of the topological evolution of MHNCs as a function of time and of systematically varied synthesis parameters, as well as on the basis of dedicated control experiments. The facile tunability of the geometric features of $\mathrm{CoPt}_{3}-\mathrm{Au}, \mathrm{FePt}-\mathrm{Au}$, and $\mathrm{Fe}_{3} \mathrm{O}_{4}-\mathrm{Ag}$ hetero-dimers can be rationalized on considering that the $\mathrm{CoPt}_{3}$ and $\mathrm{Fe}_{3} \mathrm{O}_{4}$ seeds utilized in the reported circumstances acted as red-ox active heterogeneous catalysts that exhibited varying degrees of reactivity toward reduction of the utilized $\mathrm{Au}(\mathrm{I})$ - or $\mathrm{Au}(\mathrm{III})$ - and $\mathrm{Ag}(\mathrm{I})$-ligand complex precursor, depending on their size and faceting. In the particular case of bimetallic $\mathrm{CoPt}_{3}-\mathrm{Au}$ and $\mathrm{FePt}-\mathrm{Au}$ hetero-dimers, it was ascertained that the reduction of $\mathrm{Au}(\mathrm{I})$ - or $\mathrm{Au}(\mathrm{III})$-complexes by metal species in the $\mathrm{CoPt}_{3}$ and the FePt seeds, respectively, triggerred the Au nucleation event, while subsequent growth of Au domain was fed by reduction of $\mathrm{Au}(\mathrm{I})$ or $\mathrm{Au}(\mathrm{III})$ ions at the seed surface performed by the external reducing agent (hexadecyl amine) (Krylova et al., 2012). The size to which the secondary metal domains could ultimately be grown was regulated through adjustment of seed to precursor concentration ratio and temperature, which indeed dictated the rate and extent of secondary metal-ion incorporation into the seeds (Pellegrino et al., 2006; Jiang et al., 2008; Huang et al., 2011; Peng et al., 2011; Mao et al., 2013; Zhu et al., 2013). It has been learnt that, in general, when interfacial strain is not prohibitively high, smaller-sized seeds may accommodate a continuous thick shell of the foreign materials as a means of reducing their excessive surface energy (Lee et al., 2012). On the other hand, when the seeds expose crystallographically and/or chemically inequivalent facets and/or the emergent interfacial strain, being highly dependent on surface curvature, may not be alleviated, heterodimer-type topologies are preferred over core@shell ones (Pazos-Perez et al., 2007; Jiang et al., 2008; Zhu et al., 2013).

The formation of hetero-oligomers with binary composition has frequently been reported. When faceted NCs enclosed by relatively large and stable facets with comparable surface atomic structure and/or reactivity are utilized as seeds, growth of numerous domains of the secondary components may occur on the equivalent facets or on the many surface sites (e.g., defects, edges) where nucleation may be kinetically favored and/or interfacial strain may be minimized. These circumstances may lead to heterostructures made of patchy or flower-like architectures, in which multiple satellites of the secondary material are assembled around a single central core that corresponds to one of the starting seeds (Kwon et al., 2006; McDaniel and Shim, 2009; Peng et al., 2011; Zhai et al., 2011; Hou et al., 2012; Lee et al., 2012; Mao et al., 2013; Yu et al., 2015). Depending on the specific case, the use of appropriate surface-binding surfactants (Mao et al., 2013) or judicious adjustment of the reducing conditions (Zhu et al., 2013) have been identified as effective strategies for either accentuating or leveling off the reactivity of the available seed locations, thereby modulating the frequency of heterogeneous nucleation events (Figures $\mathbf{2 H}-\mathbf{J}$ ). In another interesting study (Peng et al., 2011), during heterogeneous Ag deposition on spherical $\mathrm{Fe} @ \mathrm{Fe}_{x} \mathrm{O}_{y}$ seeds in organic media, high-order $\mathrm{Fe} @ \mathrm{Fe}_{x} \mathrm{O}_{y}-\mathrm{Ag}$ hetero-oligormers gradually converted to lower-order Fe@hollow- $\mathrm{Fe}_{x} \mathrm{O}_{y}$-Ag hetero-oligomers and, finally, to hollow- $\mathrm{Fe}_{x} \mathrm{O}_{y}-\mathrm{Ag}$ hetero-dimers (Figures $2 \mathrm{~K}-\mathbf{N}$ ). During this progression, the average Ag domain and $\mathrm{Fe}_{x} \mathrm{O}_{y}$ shell sizes increased, while the inner Fe cores of the seeds shrunk until disappearance. The time evolution of the density and size of the Ag domains was found to be consistent with the classical 


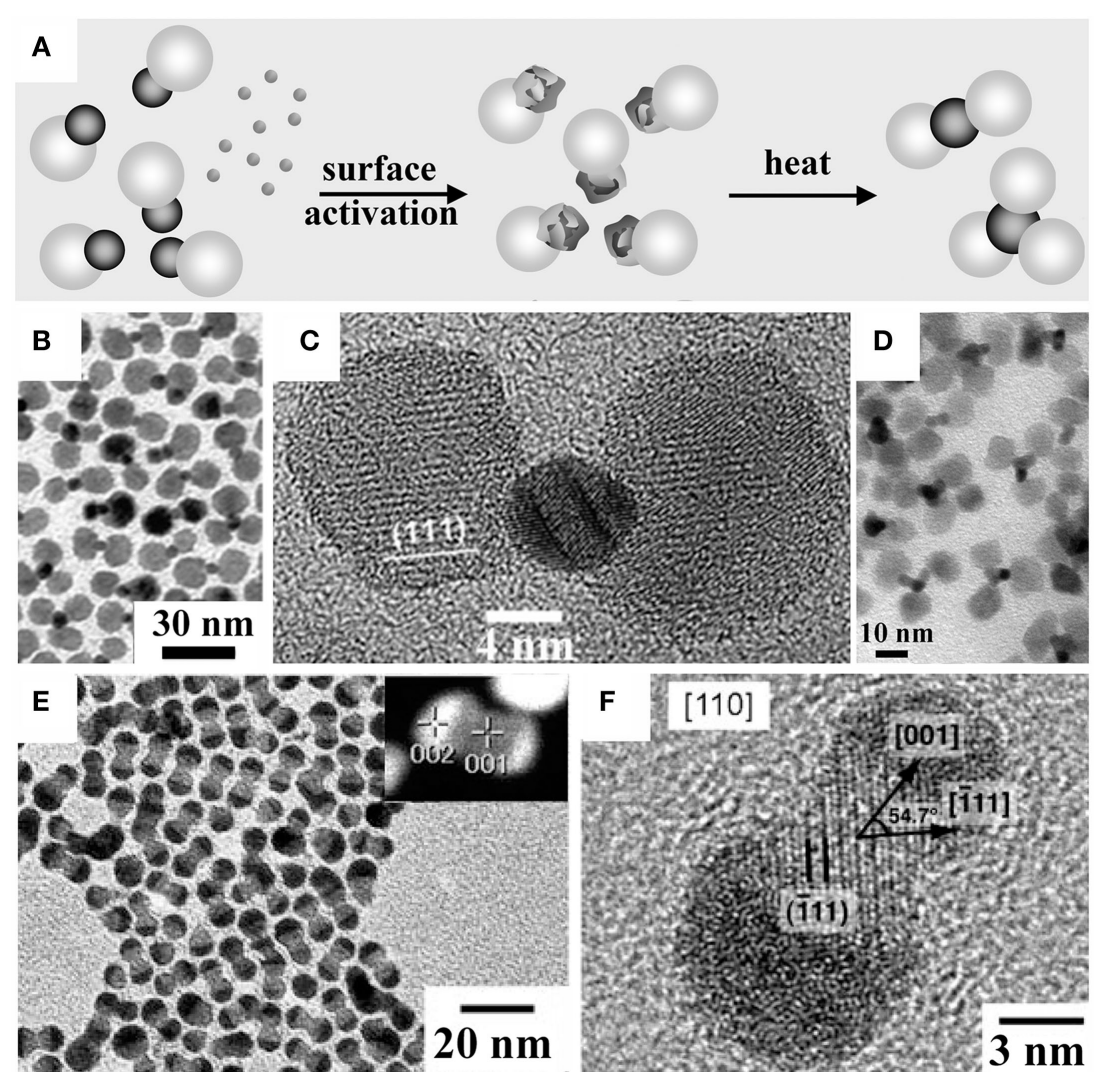

FIGURE 6 | Examples of ternary magnetic heterostructured nanocrystals (MHNCs) synthesized by induced fusion of preformed hetero-dimer seed. (A) Sketch of the mechanism [adapted from Casavola et al. (2008) with permission, copyright Wiley-VCH Verlag GmbH \& Co. KGaA]. (B-F) Low-resolution transmission electron microscopy overviews and selected high-resolution transmission electron microscopy images of: (B,C) ternary $\mathrm{Fe}_{3} \mathrm{O}_{4}-\mathrm{Au}_{-} \mathrm{Fe}_{3} \mathrm{O}_{4} \mathrm{MHNCs}_{\mathbf{H}}$ with an intermediate Au connecting domain [reproduced from Shi et al. (2006b) with permission, copyright American Chemical Society]; (D) $\left(\mathrm{Fe}_{3} \mathrm{O}_{4}-\mathrm{PtPb}\right)_{n}$ flower-like MHNCs with a central PtPb domain [reproduced from Bradley et al. (2013) with permission, copyright American Chemical Society]; (E,F) ternary PdS $S_{x}-C_{9} S_{8}-P d S_{x}$ MHNCs with a $\mathrm{Co}_{9} \mathrm{~S}_{8}$ bridging section [reproduced from Teranishi et al. (2007b), copyright Wiley-VCH Verlag GmbH \& Co. KGaA].

LaMer nucleation and Ostwald ripening mechanisms, according to which NCs are generated from a temporally limited nucleation burst, followed by the larger NCs within the population growing at the expense of the dissolution of the smaller, unstable ones. Concomitantly, the $\mathrm{Fe}$ cores of the $\mathrm{Fe} @ \mathrm{Fe}_{x} \mathrm{O}_{y}$ modules (i.e., the original seeds) underwent oxidation and entered a Kirkendall diffusion regime (An and Hyeon, 2009; Zhang et al., 2009; Wang et al., 2013b) (most likely, driven by trace $\mathrm{O}_{2}, \mathrm{Ag}^{+}$, and/or $\mathrm{NO}_{3}^{-}$ ions), which eventually led to extensive Fe dissolution and creation of hollow-Fe $\mathrm{O}_{y}$ domains with thick walls (Figure 2N) (Peng et al., 2011).

Conceptually replicating the total-synthesis approach used to build complex organic molecules equipped with different functional moieties, elaborate multicomponent hetero-oligomer MHNCs with progressively higher nuclearity order have been engineered by performing chemoselective heterogeneous nucleation steps on preformed heterostructured seeds (Figure 2A). To this purpose, well-known chemical pathways for growing the target materials were rationally combined and applied under appropriate conditions. For example, $\mathrm{Fe}_{3} \mathrm{O}_{4}-\mathrm{Au}-\mathrm{PbSe}$ heterotrimers (Figure 20) were synthesized by directing deposition of $\mathrm{PbSe}$ onto $\mathrm{Fe}_{3} \mathrm{O}_{4}-\mathrm{Au}$ dumbbell-shaped hetero-dimer seeds upon reaction with $\mathrm{Pb} / \mathrm{Se}$-surfactant complexes (Shi et al., 2006a,b).
Interestingly, the tertiary cubic-phase PbSe component grew out of the Au domains anisotropically via a solution-liquid-solid growth mechanism (Cozzoli et al., 2006), developing into rodshaped sections that eventually detached from the hetero-dimer seeds and transferred into the solution (Shi et al., 2006a). Similarly, linear $\mathrm{Fe}_{3} \mathrm{O}_{4}-\mathrm{Pt}-\mathrm{Me}(\mathrm{Me}=\mathrm{Au}, \mathrm{Ag}, \mathrm{Ni}, \mathrm{Pd})$ and $\mathrm{Fe}_{3} \mathrm{O}_{4}-\mathrm{Pt}-\mathrm{Me}_{x} \mathrm{~S}_{y}$ $(\mathrm{Me}=\mathrm{Pb}, \mathrm{Cu}$ ) hetero-trimers (Figures 2P-Q) could be constructed upon site-specific deposition of the desired tertiary metal or metal-sulfide component on the Pt domain of $\mathrm{Fe}_{3} \mathrm{O}_{4}-\mathrm{Pt}$ heterodimer seeds, a reaction that was not accompanied by the formation of other isomer side-products (Buck et al., 2012). This reaction outcome is intriguing, in that it can be regarded as a NCrelated analogue of regiospecificity in molecular systems, in which out of several products that may be formed with different spatial arrangements of their functional moieties, only one is ultimately obtained. On the basis of competently designed control experiments, the observed chemoselectivity was preliminarily assumed to correlate with an electron enrichment of the Pt domains in the $\mathrm{Fe}_{3} \mathrm{O}_{4}-\mathrm{Pt}$ hetero-dimer seeds due to charge transfer from the adjacent $\mathrm{Fe}_{3} \mathrm{O}_{4}$ domain (a process not achievable in isolated Pt nanoparticles, in fact). Further microscopic investigations of chemoselectivity in the formation of $\mathrm{Ag}-\mathrm{Pt}-\mathrm{Fe}_{3} \mathrm{O}_{4}$ hetero-trimer MHNCs (Hodges et al., 2015) revealed an initial indiscriminate 
Ag nucleation onto both the $\mathrm{Pt}$ and $\mathrm{Fe}_{3} \mathrm{O}_{4}$ surfaces of $\mathrm{Fe}_{3} \mathrm{O}_{4}-\mathrm{Pt}$ seeds, followed by surface diffusion and coalescence of $\mathrm{Ag}$ onto the Pt surface to yield the final $\mathrm{Fe}_{3} \mathrm{O}_{4}-\mathrm{Pt}-\mathrm{Ag}$ hetero-trimer product. The size of the $\mathrm{Ag}$ domain of $\mathrm{Fe}_{3} \mathrm{O}_{4}-\mathrm{Pt}-\mathrm{Ag}$ correlated with the total surface area of the $\mathrm{Fe}_{3} \mathrm{O}_{4}-\mathrm{Pt}$ seeds, which was consistent with a mechanism of Ag coalescence through a surface-mediated process. Additionally, small iron oxide islands on the Pt surface of the $\mathrm{Fe}_{3} \mathrm{O}_{4}-\mathrm{Pt}$ seeds (deposited during their synthesis) were identified as defining the morphology of the Ag domain (Hodges et al., 2015). In line with these results, further studies have been successfully extended to the case of ternary $\mathrm{Au}-\mathrm{Pt}-\mathrm{Cu}_{x} \mathrm{~S}_{y} \mathrm{HNCs}$ synthesized upon chemoselective metal-sulfide deposition over preformed Pt-Au hetero-dimers (Bradley et al., 2015).

To obtain other ternary MHNC isomers, the concept of a solidstate protecting group was borrowed from its organic-chemistry analogue and introduced into a typical colloidal MHNC synthesis (Hodges et al., 2014). A thin amorphous iron oxide shell was installed onto the $\mathrm{Pt}$ domain of preformed $\mathrm{Fe}_{3} \mathrm{O}_{4}-\mathrm{Pt}$ heterodimers to serve as a solid-state protecting group that isolated the $\mathrm{Pt}$ moiety, thus re-directing the nucleation of a third domain of Ag or $\mathrm{Au}$ to an otherwise disfavored site, namely the $\mathrm{Fe}_{3} \mathrm{O}_{4}$ domain. This strategy thus allowed producing the distinct and otherwise inaccessible $\mathrm{Ag}-\mathrm{Fe}_{3} \mathrm{O}_{4}-\mathrm{Pt}$ and $\mathrm{Au}-\mathrm{Fe}_{3} \mathrm{O}_{4}-\mathrm{Pt}$ hetero-trimer isomers, respectively.

Occasionally, heterogeneous nucleation processes have been captured to interplay and/or compete with red-ox replacement, cation exchange and the Kirkendall reaction pathways, leading to heterostructures that, however, embody an irregular distribution of their chemical composition or contain voids (Wark et al., 2008; Yang et al., 2009; Liu and Walker, 2010; Wang et al., 2013a). In the realm of MHNCs, an exception is provided by the outcome of the reaction of presynthesized metallic $\alpha$-Fe NCs with nickel acetylacetonate (Slaton et al., 2015). Mechanistic insight into this system indicated that galvanic replacement of $\mathrm{Fe}$ for Ni took place within the $\alpha$-Fe NCs, followed by rapid oxidation of both $\mathrm{Ni}$ and Fe to yield multidomain alloyed- $\mathrm{Fe}_{x} \mathrm{Ni}_{y}-\mathrm{Me}_{3} \mathrm{O}_{4}(\mathrm{Me}=\mathrm{Fe}, \mathrm{Ni})$ heterostructures.

\section{Post-Deposition Crystallization, Coalescence and Dewetting of Heterogeneosly Nucleated Shell}

Detailed investigations on the formation of heterostructures based on either $\mathrm{FePt}$ or $\gamma-\mathrm{Fe}_{2} \mathrm{O}_{3}$ and metal chalcogenides of the type $\mathrm{MeX}(\mathrm{Me}=\mathrm{Cd}, \mathrm{Zn}, \mathrm{Hg} ; \mathrm{X}=\mathrm{S}, \mathrm{Se}$ ) (Gu et al., 2004; Kwon and Shim, 2005; Kwon et al., 2006; Selvan et al., 2007; Zanella et al., 2008; He et al., 2009; McDaniel and Shim, 2009) have revealed that interfacial strain emerging upon crystallization and induced coalescence in the early post-deposition stages impacted on their topological evolution (Figure 3A). Examples of hetero-dimer and hetero-oligomer HNCs derived from these pathways are displayed in Figures 3B-E. Upon reacting either $\gamma-\mathrm{Fe}_{2} \mathrm{O}_{3}$ or FePt seeds with suitable organometallic precursors that were added sequentially at low temperature, a highly defective and amorphous MeX layer was initially deposited. Upon prolonged heating at $280^{\circ} \mathrm{C}$, the amorphous MeX shell gradually crystallized, consequently exerting strain across the formed lattice-mismatched seed/MeX heterojunctions. Over time, the shell coalesced, reshaping into a discrete MeX grain aside of the $\gamma-\mathrm{Fe}_{2} \mathrm{O}_{3}$ seed (Gu et al., 2004; Kwon and
Shim, 2005; Kwon et al., 2006; Selvan et al., 2007; Zanella et al., 2008; He et al., 2009). Such evolution was explained by considering that the large junction tension in the initially attained core@shell nanostructures could be greatly relieved during the annealing at high temperature, as supply of extra thermal energy promoted coalescence and dewetting of the crystallizing shell into a separate domain aside, which resulted in an obvious reduction of the interfacial area shared between the two materials. This interfacial energy gain could thus be large enough to compensate for the proportionally smaller increase in the overall surface energy that eventually accompanied formation of the non-core@shell heterostructure (Gentili et al., 2012; Thompson, 2012). In the case of the $\gamma-\mathrm{Fe}_{2} \mathrm{O}_{3}-\mathrm{MeX}$ system, the observed topological evolution was rationalized on the basis of the CSLT theory (Randle, 1997; Kwon and Shim, 2005; Kwon et al., 2006; McDaniel and Shim, 2009). Indeed, the mean number of MeX domains that could be accommodated on each $\gamma-\mathrm{Fe}_{2} \mathrm{O}_{3}$ seed (Figures 3B,C) correlated with the seed size and with the degree of lattice match achievable at the relevant $\gamma-\mathrm{Fe}_{2} \mathrm{O}_{3} / \mathrm{MeX}$ heterointerfaces (Kwon and Shim, 2005; Kwon et al., 2006; McDaniel and Shim, 2009). Adjustment of the ligand environment and of the growth kinetics regime (McDaniel and Shim, 2009) could facilitate installation of either one or multiple $\mathrm{CdS}$ sections that developed anisotropically out of $\gamma-\mathrm{Fe}_{2} \mathrm{O}_{3}$ seeds (Figures 3D,E). These magnetic-oxide/semiconductor MHNCs could still exhibit appreciable photoluminescence from the semiconductor MeX domains, while retaining the typical superparamagnetic behavior of nanoscale spinel-cubic iron oxide (Gu et al., 2004; Kwon and Shim, 2005; Kwon et al., 2006; Selvan et al., 2007; Zanella et al., 2008; He et al., 2009), which suggests their usefulness as bifunctional probes for dual-mode bioimaging (Selvan et al., 2007; Gao et al., 2009).

Only recently, it has been realized that post-deposition crystallization-dewetting mechanism could be involved in the formation of magneto-plasmonic hetero-dimer MHNCs more frequently than initially assumed. In fact, accurate experimental observations have allowed discrediting previous mechanistic assumptions, according to which several types of the previously developed hetero-dimer MHNCs would result from direct facetselective heterogeneous nucleation of the secondary domain onto the preexisting seeds. On the basis of the following significant reports, a mechanistic revisitation of earlier synthetic achievements should be made for the broad family of metal/iron ferrite heterostructures synthesized starting from metal seeds. The first case study that is worth mentioning concerns the formation of AuPt- $\mathrm{Fe}_{3} \mathrm{O}_{4}$ hetero-dimer MHNCs (George et al., 2011a). The starting AuPt seeds were initially captured to convert to AuPt@Fe $\mathrm{O}_{4}$ core@shell MHNCs at relatively low temperature $\left(190^{\circ} \mathrm{C}\right)$. Subsequently, under harsher conditions $\left(280-320^{\circ} \mathrm{C}\right)$, the $\mathrm{Fe}_{3} \mathrm{O}_{4}$ shell partially dewetted and restructured into a discrete spherical $\mathrm{Fe}_{3} \mathrm{O}_{4}$ domain attached aside each AuPt seed core. Along this process, the AuPt domain retained a thin discontinuous $\mathrm{Fe}_{3} \mathrm{O}_{4}$ shell on the hemispherical region that was diametrically opposite to the location of the major coalesced $\mathrm{Fe}_{3} \mathrm{O}_{4}$ domain. A similar mechanism relying on the dewetting of an unstable thin $\mathrm{Fe}_{3} \mathrm{O}_{4}$ shell formed on the metal seeds at early to intermediate stages may thus be expected to be involved in the formation of other $\mathrm{Me}-\mathrm{Fe}_{3} \mathrm{O}_{4}(\mathrm{Me}=\mathrm{Au}, \mathrm{AuPt})$ hetero-dimers. 
Actually, interesting "tug-of-war" etching-destabilization experiments, aimed at probing the chemical reactivity of these heterostructures (Wang et al., 2009b; Lee et al., 2010b; George et al., 2011a), have shown that Au could be selectively leached out from $\mathrm{Me}-\mathrm{Fe}_{3} \mathrm{O}_{4}$ hetero-dimers upon oxidation with $\mathrm{I}_{2}$ at near room temperature. Depending on the topological profile of the starting hetero-dimers, either nearly spherical $\mathrm{Fe}_{3} \mathrm{O}_{4} \mathrm{NCs}$, each bearing a concavity, or peanut-/dumbbell-like solid-hollow homo-dimer $\mathrm{Fe}_{3} \mathrm{O}_{4} \mathrm{NCs}$ were obtained. In all cases, the formed concave region or void was exactly commensurate to the volume that was originally occupied by the Au domain; in addition, in the particular case of $\mathrm{AuPt}-\mathrm{Fe}_{3} \mathrm{O}_{4}, \mathrm{I}_{2}$-driven oxidation of the AuPt domain led to a nanocontainer section that encased a residual Pt domain (George et al., 2011a). The formation of such exotic nanostructures with concave surfaces or extended cavities indirectly discredited the earlier assumption that the metal (hemi)domain of these hetero-dimer MHNCs was totally "naked," that is, in direct contact with the external environment; in contrast, the metaletching experiments unveiled that the portion of the metal, which was not nested in the $\mathrm{Fe}_{3} \mathrm{O}_{4}$ domain, could accommodate a thin, porous (hence, permeable), or discontinuous $\mathrm{Fe}_{3} \mathrm{O}_{4}$ shell that guaranteed its chemical accessibility, a feature that should be taken into account to explain their (electro)catalytic performance (Yin et al., 2008; Wang et al., 2009a, 2010; Wu et al., 2009; Lee et al., 2010b; George et al., 2011a, 2013; Lin and Doong, 2011; Chen et al., 2012; Sun et al., 2012).

A very recent detailed study has unexpectedly revealed participation of dewetting also in the formation of all-metallic $\mathrm{Au}-\mathrm{Pt}$ alloy hetero-dimer MHNCs (Kwon et al., 2015). According to this report, heteroepitaxial deposition of Au on the Pt-alloy seeds proceeded through the initial formation of a thin $\mathrm{Au}$ shell that exerted high stress onto the seed underneath. In the subsequent reaction stage, the lattice strain intensified to the point of inducing Au dewetting, a process that, in turn, allowed for strain relaxation. Thus, the Au/Pt-alloy heterostructures underwent a topological transition from a core@shell to a dumbbell-like configuration.

\section{Heterogeneous Nucleation at Liquid/Liquid Interfaces between Immiscible Solvents}

An interesting technique to synthesize magneto-plasmonic hetero-dimer MHNCs relies on performing the deposition of a secondary metal component on seeds located at the liquid/liquid interface between immiscible liquid phases (Figure 4A). Examples of MHNCs derived by this strategy are shown in Figures 4B-G. In the reported procedure, an aqueous solution containing a $\mathrm{Au}(\mathrm{III})$ or $\mathrm{Ag}(\mathrm{I})$ salt was put in contact with an immiscible organic phase that contained surfactant-capped $\mathrm{Fe}_{x} \mathrm{O}_{y}$ or $\mathrm{FePt}$ seeds dissolved in a non-polar solvent, such as dichlorobenzene, dichloromethane, hexane, or DOE (Gu et al., 2005; Pan et al., 2010). Upon ultrasound irradiation under inert atmosphere, an emulsion formed, which consisted of a continuous aqueous phase containing "colloidosomes," organic microdroplets stabilized by the hydrophobic-capped seed NCs that had self-assembled at the organic/water interfaces (Dinsmore et al., 2002). Under these conditions, the seeds provided catalytically active sites onto which the $\mathrm{Ag}^{+}$or $\mathrm{AuCl}_{4}^{-}$ions were reduced to the respective metals during the ultrasonication ( $\mathrm{Gu}$ et al., 2005). As the seeds were only partially exposed to the aqueous phase, metal deposition was spatially restricted to a small region of their surface and proceeded self-catalytically, thus allowing the formation of only one metal domain on each seed (Figures 4B-E). The "colloidosome"-based approach was extended to the synthesis of solid-Ag/hollow- $\boldsymbol{\gamma}-\mathrm{Fe}_{2} \mathrm{O}_{3}$ hetero-dimer MHNCs (Figures 4F,G) starting from hollow $\gamma-\mathrm{Fe}_{2} \mathrm{O}_{3}$ seeds prepared by manipulating the Kirkendall mechanism (An and Hyeon, 2009; Zhang et al., 2009; Pan et al., 2010; Wang et al., 2013b; Qi et al., 2015). These MHNCs were used to accommodate a site-differential surface distribution of biomolecules that were exploitable for biomedical purposes (An and Hyeon, 2009; Gao et al., 2009; Pan et al., 2010).

\section{Self-Regulated Homogeneous and Heterogeneous Nucleation in the Absence of Presynthesized Seeds}

A few reports have documented the synthesis of hetero-dimer MHNCs by one-pot approaches that do not involve the introduction of seeds presynthesized in a separate environment (Figure 5A). In these cases, all reagents required to construct the heterostructures are loaded together into the same solvent/surfactant medium since the beginning of the synthesis. Conditions may be serendipitously identified under which the specific nucleation and growth processes that underlie the formation of each different material module require distinct activation energies and are thus triggered at substantially different rates, thus proceeding in sequence with negligible temporal overalap (Erdemir et al., 2009). Liquid-cell TEM imaging, a technique that has very recently been advanced to the point of allowing NC evolution to be tracked directly in solution in real time (Ross, 2015), has unambiguously confirmed that self-regulated homogeneous and heterogenous nucleation dynamics can indeed be operative in the formation of MHNCs (Liang et al., 2015). Figures 5B-E collects examples of MHNCs derived by such pathways.

$\mathrm{Co}_{9} \mathrm{~S}_{8}-\mathrm{PdS}_{x}$ hetero-dimer MHNCs with acorn profile (Figures 5A,B) were produced by co-pyrolysis of the corresponding cobalt and copper carboxylate precursors in the presence of alkylthiols as the sulfur source (Teranishi et al., 2004, 2007a). As these metal sulfides are only partially miscible and feature large interfacial energy, a solid-solution could not be created, whereas phase segregation of two materials took place instead. During the reaction, selective homogeneous nucleation and growth of one material did occur first; then, as the critical miscibility threshold was reached, the second component started to develop, progressing through an interface of graded composition (Erdemir et al., 2009). Similarly, Au-Ni hetero-dimer MHNCs (Figure 5C) were obtained upon heating $\mathrm{HAuCl}_{4}$ and $\mathrm{Ni}\left(\mathrm{NO}_{3}\right)_{2}$ in octadecyl amine (ODA) at $120^{\circ} \mathrm{C}$, during which initially generated $\mathrm{Au}$ seeds triggered the otherwise kinetically hindered reduction of $\mathrm{Ni}^{2+}$-ODA complexes at their surface (Wang and Li, 2010).

Another interesting case is represented by the evolution of $\mathrm{FePt}-\mathrm{Fe}_{\mathrm{x}} \mathrm{O}_{\mathrm{y}}$ hetero-dimers for which independent domain size tunability could be easily achieved (Figure 5D). The two material modules formed sequentially in two steps during the reaction of platinum acetylacetonate and $\mathrm{Fe}(\mathrm{CO})_{5}$ in hot OLAM/OLAC/ODE mixtures (Figuerola et al., 2008). Initially, homogeneous nucleation and growth of FePt NCs were allowed to take place at 
lower temperature $\left(T \approx 180-200^{\circ} \mathrm{C}\right)$; then, a thin polycrystalline $\mathrm{Fe}_{x} \mathrm{O}_{y}$ shell was deposited onto the in situ generated FePt seeds upon prolonged heating of the reaction mixture at $T \approx 295^{\circ} \mathrm{C}$. The shell rapidly dewetted out and evolved into a separate cubic spinel $\mathrm{Fe}_{3} \mathrm{O}_{4} @ \gamma-\mathrm{Fe}_{2} \mathrm{O}_{3}$ core@shell domain attached aside, leaving a thin iron oxide shell on the diametrically opposite side of FePt (Nolle et al., 2009). Overall, this process permitted alleviating the intervening interfacial strain due to large difference $(\sim 8 \%)$ in lattice parameters between $\mathrm{FePt}$ and iron oxide (Erdemir et al., 2009; Nolle et al., 2009; Gentili et al., 2012; Thompson, 2012), albeit at the cost of nucleation of dislocations within the heterodimer lattice (Figuerola et al., 2008). Since each reaction step was selectively activated under distinct thermal conditions, regulation of both the temperature and the heating time guaranteed that the two material sections of the MHNCs formed at different stages of the synthesis course (Erdemir et al., 2009). As a consequence of the magnetic exchange coupling holding between the two soft and hard materials, the $\mathrm{FePt}-\mathrm{Fe}_{x} \mathrm{O}_{y}$ MHNCs exhibited tunable single-phase-like magnetic behavior and superior performance as MRI contrast agents, not otherwise achievable by their individual components (Figuerola et al., 2008; Nolle et al., 2009).

In a later report, OLAM-driven thermal reduction of rhodium acetate and decomposition of iron acetylacetonate were accomplished in consecutive heating steps at $200^{\circ} \mathrm{C}$ and $300^{\circ} \mathrm{C}$, respectively, to synthesize asymmetric peanut-shaped $\mathrm{Rh}-\mathrm{Fe}_{3} \mathrm{O}_{4}$ heterodimer MHNCs in OLAM/OLAC media (Jang et al., 2011b). In these heterostructures, the tiny $\mathrm{Rh}$ domain exhibited excellent iron-oxide-promoted catalytic activities for the selective reduction of nitroarenes and alkenes, while the larger supporting iron oxide allowed facile magnetic recyclability.

\section{Induced Attachment of Preformed MHNCs}

A total-synthesis framework with the potential to access increasingly complex MHNCs envisions utilization of smaller, low-order MHNCs as inorganic precursors equipped with functional moieties that can be forced to react, stick, and fuse, thereby leading to larger, higher-order MHNCs (Figure 6A). This synthetic scheme that conceptually mimics molecular coupling or condensation reactions in the organic-chemistry toolkit, has earlier been exploited for the creation of corresponding prototypes of allpolymeric nano- and microparticle clusters (Duguet et al., 2011; Vogel et al., 2015). Examples of all-inorganic MHNCs obtained by exploitation of this strategy are shown in Figures 6B-F. $\mathrm{Fe}_{3} \mathrm{O}_{4}-\mathrm{Au}-\mathrm{Fe}_{3} \mathrm{O}_{4}$ hetero-trimer MHNCs, in which a Au section bridged two $\mathrm{Fe}_{3} \mathrm{O}_{4}$ domains, were obtained by inducing welding of the $\mathrm{Au}$ domains that belonged to distinct peanut-shaped $\mathrm{Au}-\mathrm{Fe}_{3} \mathrm{O}_{4}$ hetero-dimers under the assistance of added elemental sulfur (Shi et al., 2006b). Due to its high affinity to Au surfaces, $\mathrm{S}$ was presumed to adsorb on the $\mathrm{Au}$ of the heterodimers and to facilitate displacement of the capping surfactants thereon, thus making the $\mathrm{Au}$ sections prone to fuse with each other to lower their total surface energy (Figures 6B,C). Further elaboration of this strategy enabled access to higherorder hetero-oligomers with linear or variably branched topology. For example, $\mathrm{Fe}_{3} \mathrm{O}_{4}-\mathrm{Pt}-\mathrm{Au}-\mathrm{Au}-\mathrm{Pt}-\mathrm{Fe}_{3} \mathrm{O}_{4}$ MHNCs with different three-dimensional connectivity were synthesized upon sulfur-participated selective coupling of preformed $\mathrm{Au}-\mathrm{Pt}-\mathrm{Fe}_{3} \mathrm{O}_{4}$ hetero-trimers through their Au domains (Buck et al., 2012). In another account, preformed $\mathrm{PtPb}-\mathrm{Fe}_{3} \mathrm{O}_{4}$ hetero-dimers were observed to spontaneously aggregate, upon aging at room temperature in hexane, into multinuclear $\left(\mathrm{PtPb}-\mathrm{Fe}_{3} \mathrm{O}_{4}\right)_{n}$ flower-like heterostructures (Figure 6D), each resulting from the congregation of several $\mathrm{PtPb}-\mathrm{Fe}_{3} \mathrm{O}_{4}$ hetero-dimers via their $\mathrm{PtPb}$ domains (Bradley et al., 2013).

In another report, peanut-shaped $\mathrm{PdS}_{x}-\mathrm{Co}_{9} \mathrm{~S}_{8}-\mathrm{PdS}_{x}$ MHNCs, in which a central $\mathrm{Co}_{9} \mathrm{~S}_{8}$ section connected two $\mathrm{PdS}_{x}$ domains (Figures 6E,F), were synthesized by reacting $\mathrm{PdS}_{x}$ seeds with cobalt acetate and octadecanthiol precursors in DOE at $230^{\circ} \mathrm{C}$ (Teranishi et al., 2007a,b). As demonstrated by time monitoring of the reaction course, the ternary $\mathrm{PdS}_{x}-\mathrm{Co}_{9} \mathrm{~S}_{8}-\mathrm{PdS}_{x}$ MHNCs evolved upon crystal-oriented coalescence of acornshaped $\mathrm{PdS}_{x}-\mathrm{Co}_{9} \mathrm{~S}_{8}$ hetero-dimers that had been generated in the earlier reaction stages. The process appeared to be promoted by the weak organic passivation of the $\mathrm{Co}_{9} \mathrm{~S}_{8}$ domains, which made them inclined to weld with each other.

\section{Heterostructures Based on Anisotropically Shaped Material Modules}

MHNCs with a spatially asymmetric arrangement of their component modules have been created by manipulating heterogeneous nucleation/growth reactions on anisotropically shaped seeds, such as nanorods, nanowires, and branched NCs (Cozzoli et al., 2006; Jun et al., 2006). In addition to offering peculiar shape-dependent properties, anisotropic seeds normally exhibit a pronounced facetdependent reactivity, thus representing interesting model platforms over which pathways to site-selective implantation of foreign domains can be investigated and rationalized. On a thermodynamic basis, the preference for secondary material deposition to take place at given locations of anisotropic seeds should correspond to the lowest-energy topological configuration, among all those allowed by the surface-interface energy variation accompanying the formation of all relevant heterointerfaces (Eq. 1) (Cozzoli et al., 2006; Jun et al., 2006; Costi et al., 2010; de Mello Donegà, 2011; Banin et al., 2014). For example, selective heterogeneous nucleation may be viewed as being energetically convenient when it permits elimination of some unstable facets of the seeds (e.g., high-index facets, located, for example, at the apexes or edge regions of nanorods) at the proportionally smaller cost of the interfacial strain energy spent in the formation of the heterojunctions. However, when nucleation and growth occur under kinetically controlled regimes, and/or other chemical or physical transformative pathways come into play (e.g., intraparticle ripening, atomic diffusion, and ion exchange), thermodynamic predictions may not be verified and thus the comprehension of the mechanisms underlying topology selection may be far less straightforward (Cozzoli et al., 2006; Jun et al., 2006; Casavola et al., 2007, 2008; Wetz et al., 2007; Maynadiè et al., 2009; Costi et al., 2010; de Mello Donegà, 2011; Banin et al., 2014).

The following paragraphs describe the major synthetic strategies that have been devised to construct MHNCs that entail anisotropic (linear or branched) material sections. The main growth mechanisms that have been exploited include: (i) regioselective heterogeneous nucleation/growth governed by the 


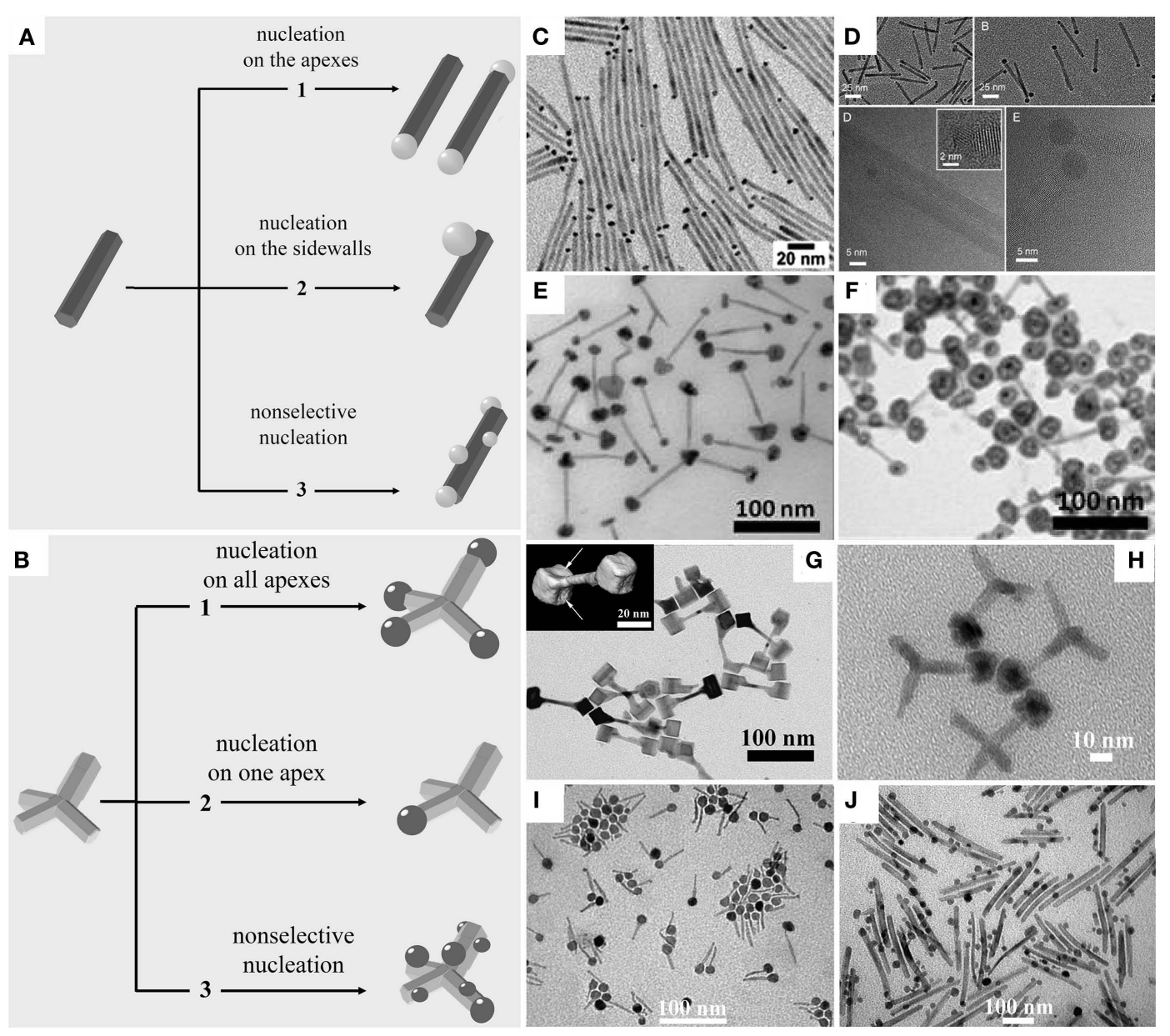

FIGURE 7 | Examples of anisotropic magnetic heterostructured nanocrystals (MHNCs) synthesized by regioselective heterogeneous deposition of secondary materials domains on preformed nanorod and tetrapod seeds. (A,B) Sketch of the mechanism. (C-J) Low-resolution transmission electron microscopy images of: (C) nanodumbbells of NiPt-tipped CdS nanorods [reproduced from Habas et al. (2008) with permission, copyright American Chemical Society]; (D) nanodumbbells of Ni-tipped CdS nanorods [reproduced from Nakibli and Amirav (2016) with permission, copyright American Chemical Society]; (F) nanodumbbells of Pt@Co-tipped CdS nanorods obtained upon Co deposition on the tips of preformed Pt-tipped CdS nanorods and (E) nanodumbbells of corresponding Pt@Co $\mathrm{O}_{y}$-tipped CdS nanorods with Pt@Co $\mathrm{O}_{y}$ yolk@shell termini obtained upon Co oxidation of their parent Pt@Co-tipped CdS nanorods [reproduced from Hill et al. (2012) with permission, copyright American Chemical Society]; (G) nanodumbbells of Co nanorods bearing concave Fe cubes at the apexes, along with (see inset) a tomographic reconstruction of a single heterostructure, where arrows indicate concave surfaces on the terminal Fe cubes [reproduced from Liakakos et al. (2014) with permission; copyright American Chemical Society]; (H) CdSe@CdS core@shell tetrapods asymmetrically decorated with a single Au@Co core@shell tip domain [reproduced with permission from Pavlopoulos et al. (2016), copyright Wiley-VCH Verlag GmbH \& Co. KGaA]; (I) nanomatchsticks made of single- $\gamma$ - $\mathrm{Fe}_{2} \mathrm{O}_{3}$-tipped brookite $\mathrm{TiO}_{2}$ nanorods [reproduced from Buonsanti et al. (2010) with permission, copyright American Chemical Society]; ( $\mathbf{J}$ ) hetero-oligomers made of multiply $\gamma-\mathrm{Fe}_{2} \mathrm{O}_{3}$-decorated brookite $\mathrm{TiO}_{2}$ nanorods [adapted from Buonsanti et al. (2009) with permission from the PCCP Owner Societies].

inherent structure and reactivity of the seeds; (ii) surfactantcontrolled regioselective heterogeneous deposition; and (iii) strain-driven heteroepitaxial growth. Examples that demonstrate the degree of synthetic control achievable by these pathways can be found in Figures 7-9.

\section{Facet-Controlled Regioselective Heterogeneous Nucleation and Growth}

The most developed breeds of anisotropically shaped HNCs are represented by polytypic nanoheterostructures based on the archetypal II-VI metal-chalcogenide compounds of the type MeX $(\mathrm{Me}=\mathrm{Cd}, \mathrm{Zn}, \mathrm{Mn} ; \mathrm{X}=\mathrm{S}, \mathrm{Se})$ and on transition-metal oxides (such as $\mathrm{ZnO}, \mathrm{TiO}_{2}$ ), for which refined synthetic capabilities have been mastered over the past two decades (Cozzoli et al., 2006; Jun et al., 2006; Li et al., 2013; Sitt et al., 2013). Nanoplatelets, nanorods, nanowires, and polypods of these materials commonly crystallize in low-symmetric crystal phase (e.g., hexagonal, tetragonal, and orthorhombic) that preferentially extend along or perpendicular to their axis of higher symmetry. Shape anisotropy has important consequences on the seeding behavior of these NCs when used in seed-mediated syntheses. The facets at the apex, edges, and longitudinal/basal sidewalls generally feature distinct atomic arrangement, hence dissimilar chemical reactivity. In nanorods/wires lacking a plane of symmetry perpendicular to their major axis (e.g., hexagonal wurtzite), the two terminal basal facets at the extremities are crystallographically, hence chemically, 


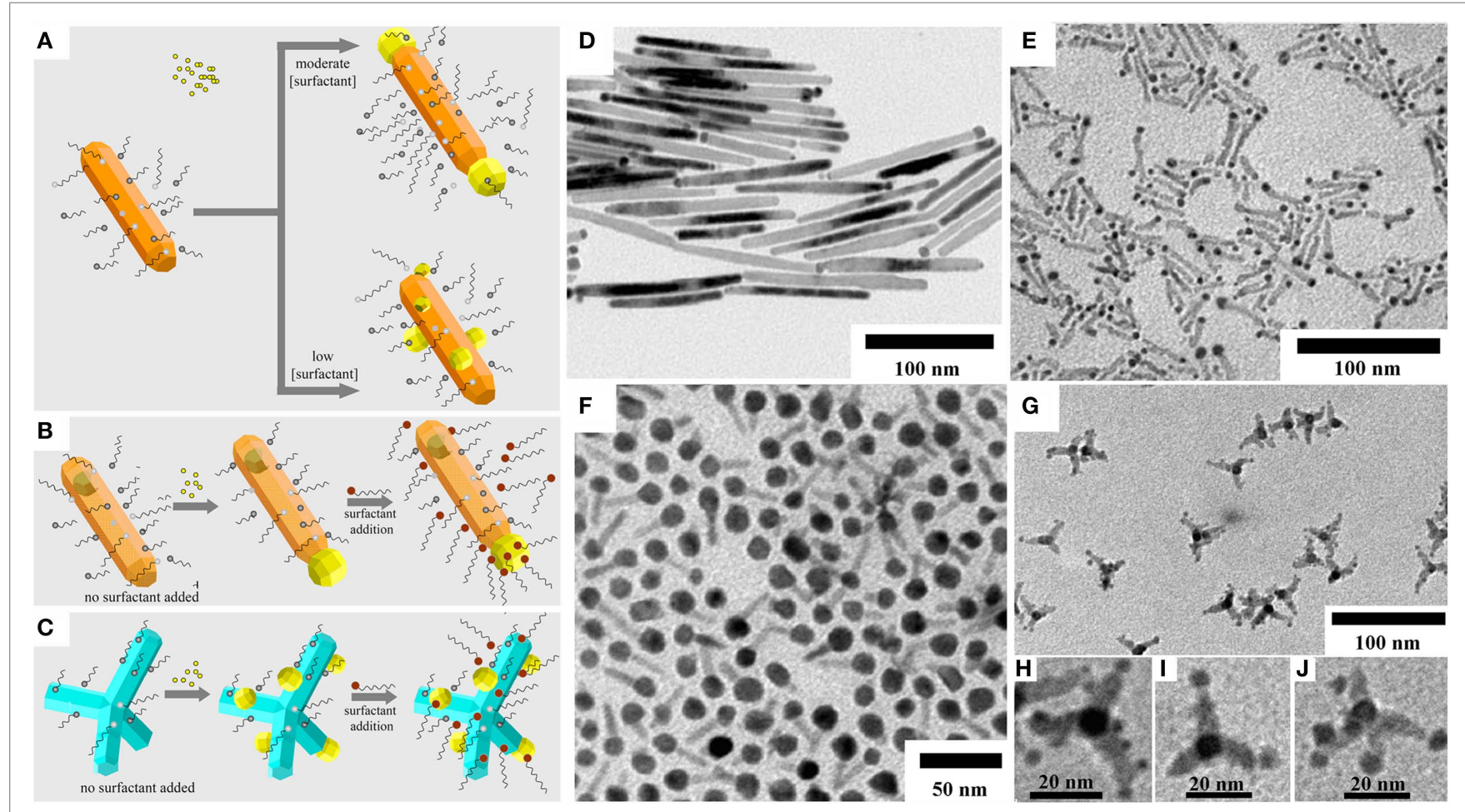

FIGURE 8 | Examples of anisotropic magnetic heterostructured nanocrystals (MHNCs) synthesized by surfactant-controlled regioselective heterogeneous nucleation on preformed nanorod and branched seeds. (A-C) Sketch of the mechanism [adapted from Carbone and Cozzoli (2010), copyright 2010, with permission from Elsevier]; (D-J) Low-magnification transmission electron microscopy galleries of: (D) dumbbell-like heterostructured nanocrystals (HNCs) made of Au-tipped Co nanorods [reproduced from Wetz et al. (2007) with permission, copyright Wiley-VCH Verlag GmbH \& Co. KGaA]; (E) dumbbell-like and matchstick-like Co-tipped anatase $\mathrm{TiO}_{2}$ nanorods [reproduced from Casavola et al. (2007) with permission, copyright American Chemical Society]; (F) matchstick-like MHNCs made of eccentric CdSe@CdS nanorods tipped with a single Co head [synthesized according to Deka et al. (2009)]; (G-J) HNCs made of multiply Co-decorated $\gamma-\mathrm{Fe}_{2} \mathrm{O}_{3}$ tetrapods [reproduced with permission from Casavola et al. (2009), copyright American Chemical Society].

non-equivalent. Arrowing or tapering at the nanorod termini may further exacerbate such dissimilarities. In line with the mechanism of their anisotropic growth, the longitudinal sidewalls and the tips of nanorods/nanowires can be expected to exhibit significantly different propensity to accommodate secondary material domains (Cozzoli et al., 2006; Casavola et al., 2008; Carbone and Cozzoli, 2010; Costi et al., 2010; de Mello Donegà, 2011; Sitt et al., 2013; Banin et al., 2014; Xu et al., 2015) in seeded-growth synthesis (Figure 7A). Similarly, the apexes and the longitudinal sidewalls of the arms sections of branched NCs (Li et al., 2013) can be expected to exhibit distinct reactivity behavior (Figure 7B). Furthermore, depending on the specific case, shaped NCs, which have formed with a lattice deviating from the ideal bulk crystallographic structure, or, which have been configured with an inner core@shell architecture, can hold an intrinsic dipole moment or accommodate defective or strained near sub-surface regions, which may influence their seeding capabilities (Menagen et al., 2008; Deka et al., 2009; Costi et al., 2010; Li et al., 2013; Banin et al., 2014).

At the relevant bonding heterointerfaces, the mismatched lattice arrangement can break the crystal periodicity to a varying extent, giving rise to noticleable interfacial strain, which may, in turn, affect their electronic-band structure (Costi et al., 2010; Sitt et al., 2013; Banin et al., 2014; Xu et al., 2015) as well as the type and extent of exchange coupling between different magnetic phases or between the optical and magnetic functionalities
(Casavola et al., 2007, 2009; Wetz et al., 2007; Deka et al., 2009; Maynadiè et al., 2009; Liakakos et al., 2014). Interfacial misfit strain may be alleviated by different pathways. For example, at the high temperatures at which colloidal synthesis is typically carried out, the overgrown material may adapt to the lattice parameters of the seed at the interface (coherency strain) (Dunstan, 1997; Chen et al., 2003; Xu et al., 2015). In other circumstances, the constituent atomic species of the connected materials may interdiffuse, accommodating variation in the chemical composition and/or crystal-lattice parameters across the heterojunction regions (Koo and Korgel, 2008; Yuhas et al., 2009). In the extreme cases of exceedingly large misfit, plastic relaxation may induce formation of interfacial defects, such as dislocations and stacking faults (Dunstan, 1997; Markov, 2003; Carbone and Cozzoli, 2010; Li et al., 2014). Experimental results (Casavola et al., 2007, 2009; Koo and Korgel, 2008; Deka et al., 2009; Yuhas et al., 2009; Liakakos et al., 2014) and thermodynamic modeling (Sadowski and Ramprasad, 2010; Xu et al., 2015) have indicated that the temperature and the particular chemical pathways underlying MHNC formation are decisive in determining whether either uniformly alloyed, graded, or abrupt interfaces may be attained.

As for their core@shell counterparts, in anisotropic MHNCs based on associations of dissimilar semiconductors, (magnetic) metals and metal oxides, the energy level structure 


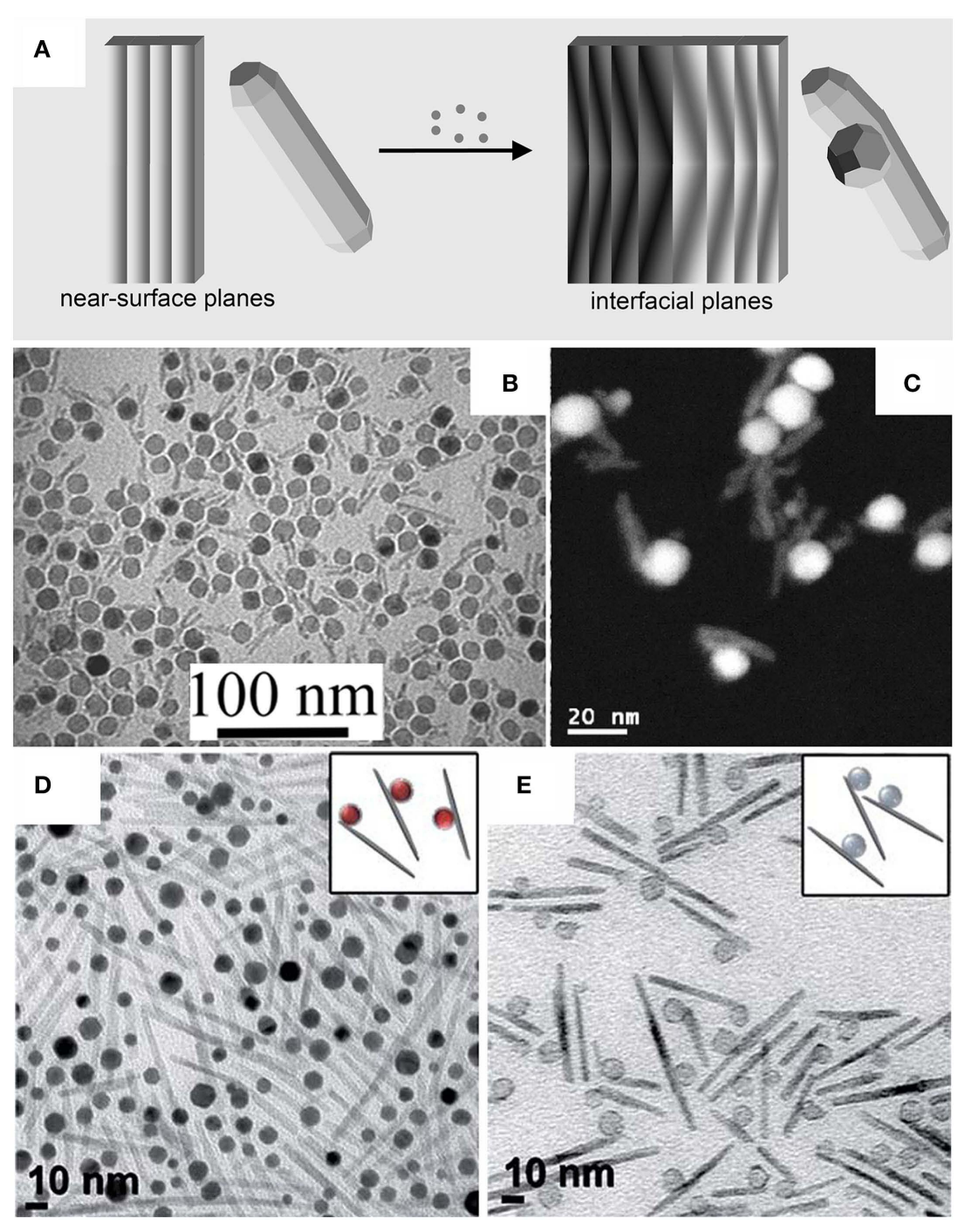

FIGURE 9 | Examples of magnetic heterostructured nanocrystals formed upon strain-driven heteroepitaxial growth. (A) Sketch of the mechanism [adapted from Casavola et al. (2008) with permission, copyright Wiley-VCH Verlag GmbH \& Co. KGaA]. (B,C) Low-resolution transmission electron microscopy (TEM) image and high-angle annular dark-field image in scanning transmission electron microscopy mode, respectively, of binary heterostructures made of single- $\gamma-\mathrm{Fe}_{2} \mathrm{O}_{3}$-functionalzied anatase $\mathrm{TiO}_{2}$ nanorods [reproduced with permission from Buonsanti et al. (2006), copyright American Chemical Society]; (D) TEM images of binary heterostructures made of asymmetrically Au@Fe $\mathrm{O}_{y}$-decorated $\mathrm{Fe}_{x} \mathrm{O}_{y}$ nanorods; and (E) corresponding all-Fe $\mathrm{O}_{y}$ nanostructures obtained after Au leaching [reproduced from George et al. (2011b) with permission from The Royal Society of Chemistry].

across the interface can be purposely engineered upon proper heterostructure design, so as to promote confinement or spatial separation of the photogenerated charge carriers (Costi et al., 2010; de Mello Donegà, 2011; Sitt et al., 2013; Banin et al., 2014; Xu et al., 2015). Interestingly, the overall magnetic behavior may also result in being remarkably affected, even when magnetic modules are connected to non-magnetic materials (Casavola et al., 2007, 2009; Wetz et al., 2007; Deka et al., 2009; Maynadiè et al., 2009; Liakakos et al., 2014). Such hybrid nanoarchitectures hold promise as new functional elements for the realization of optoelectronic devices and efficient magnetically recoverable photo(electro)catalytic platforms (Costi et al., 2010; de Mello Donegà, 2011; Sitt et al., 2013; Banin et al., 2014), spintronic devices, and magnetic recording systems (Casavola et al., 2007,
2009; Wetz et al., 2007; Deka et al., 2009; Maynadiè et al., 2009; Liakakos et al., 2014).

Demonstration of all the synthesis concepts and structureproperty relations summarized above is provided in numerous accounts illustrating the colloidal fabrication and characterization of MHNCs with matchstick-like, dumbbell-like, and branched topologies, in which either one or both apexes, or the longitudinal sidewalls of nanorod (arm) sections of the seeds are decorated with domains of other materials. Representative examples are reported in Figures 7C-J.

Intriguing breeds of MHNCs obtained by the heterogeneous nucleation/growth are represented by semiconductor-metal heterostructures, whereby anisotropic semiconductor NCs have been exploited as underlying substrates for accommodating various 
metal domains. Standard pyrolytic organometallic routes were suitably combined and utilized to synthesize $\mathrm{CdX}-\mathrm{Me}$ and Me-CdX-Me heterostructructures (Figures 7C,D) based on CdX $(\mathrm{X}=\mathrm{Se}, \mathrm{S})$ nanorods functionalized with magnetic and nonmagnetic domains $(\mathrm{Me}=\mathrm{Au}, \mathrm{Pt}, \mathrm{PtNi}, \mathrm{PtCo}, \mathrm{Co}, \mathrm{Ni}$ ) (Mokari et al., 2004, 2005; Habas et al., 2008; Jen-La Plante et al., 2009; Maynadiè et al., 2009; Yuhas et al., 2009; Nakibli and Amirav, 2016). For these systems, a high degree of selectivity for metal nucleation at both seed apexes was readily achievable (Figure 7A, path 1) (Habas et al., 2008; Jen-La Plante et al., 2009; Wang et al., 2009d; Nakibli and Amirav, 2016), whereas indiscriminate deposition at random sites was observed when large aspect-ratio nanorods/nanowires were used as starting seeds, or when the synthesis was carried out at exceedingly high reactant-to-seed concentration ratios (Figure 7A, path 3). However, within the context of delicately manipulated organometallic chemistry, the choice of coordinating ligands and adjustment of their concentration in the liquid media appeared to be critical to ensuring topological selectivity, disclosing the important role of ligandcontrolled kinetic processes (Habas et al., 2008; Maynadiè et al., 2009; Hill et al., 2012; Nakibli and Amirav, 2016).

As an effort toward increased structural and compositional complexity, secondary metal domains on preformed heterostructures were envisaged to be exploitable as highly reactive sites onto which exclusive deposition of a third component could be steered. This idea has actually been proven to be practically feasible within the framework of rationally designed multiseeding-step reaction schemes (Hill et al., 2012; Pavlopoulos et al., 2016). In one report, Pt-tipped Cd@CdS core@shell nanorods were exploited to direct the otherwise unfeasible overgrowth of Co onto the Pt tips leading to corresponding Pt@Co-CdSe@CdS-Pt@Co dumbbell-like MHNCswithPt@Co core@shell terminal domains; these MHNCs could be then converted into Pt@ $\mathrm{Co}_{x} \mathrm{O}_{y}-\mathrm{CdSe} @ \mathrm{CdS}-\mathrm{Pt} @ \mathrm{Co}_{x} \mathrm{O}_{y}$ dumbbell-like heterostructures with $\mathrm{Pt} @ \mathrm{Co}_{x} \mathrm{O}_{y}$ yolk@shell termini (Figures 7E,F) upon selective oxidation and hollowing of the Co shell of the Pt@Co apexes (Hill et al., 2012). In another protocol (Pavlopoulos et al., 2016), asymmetrically Au-tipped CdSe@CdS tetrapods were first fabricated by accommodating one single $\mathrm{Au}$ sphere at one of the four arm termini of preformed Cd@CdS core@shell tetrapods through a photodeposition process that proceeded through the initial attainment of multiply Au-decorated tetrapod arms, followed by intraparticle ripening (Mokari et al., 2005) of the as-nucleated $\mathrm{Au}$ patches into a single $\mathrm{Au}$ tip (Figure 7B, paths 1 and 2); subsequently, thermolysis of $\mathrm{Co}_{2}(\mathrm{CO})_{8}$ in the presence of the Au-CdSe@CdS tetrapod heterostructures and of carboxylic-acid-terminated polystyrene ligands in 1,2,4trichlorobenzene at $T=140^{\circ} \mathrm{C}$ enabled selective overgrowth of a Co shell around the previously implanted Au domains. As a result, MHNCs made of CdSe@CdS core@shell tetrapods asymmetrically decorated with a singleAu@Co core@shell domain at one apex were obtained (Figure $7 \mathbf{H}$ ).

For all of the aforementioned semiconductor-based MHNCs, the strong electronic-band coupling between the semiconductor and the magnetic-metal sections was corroborated by a significant luminescence quenching, which transcribed into increased charge-carrier separating capabilities (Costi et al., 2008, 2010; Sitt et al., 2013; Banin et al., 2014). However, localized surface states at the relevant interfaces were also invoked to account for the dramatically altered optoelectronic properties of the MHNCs (Steiner et al., 2005; Costi et al., 2008, 2010; Jen-La Plante et al., 2009; Sitt et al., 2013; Banin et al., 2014). Depending on the reaction medium (e.g., the presence of hole/electron scavengers) and the irradiation conditions, the metal section could either act a sink for the electrons photogenerated within the semiconductor, or help transferring them to the solution. Manipulation of these mechanisms enabled exploitation of these MHNCs as photocatalysts capable of performing efficient charge retention or affording enhanced photocatalytic reduction yields, respectively (Costi et al., 2008, 2010; Elmalem et al., 2008; Amirav and Alivisatos, 2010; Sitt et al., 2013; Banin et al., 2014; Nakibli and Amirav, 2016). The magnetic-metal domains could also be utilized to enable magnetic separation and subsequent recycling of the photocatalysts. The inherent magnetic properties were also found to be modified upon heterojunction attainment (Jen-La Plante et al., 2009; Maynadiè et al., 2009). Interestingly, selective anchoring of magnetic-metal sections onto shaped semiconductor NCs was proven to be a valuable strategy toward promoting the self-assembly of corresponding anisotropic MHNCs into nanoheterostructure "polymers" by profiting from tip-to-tip dipolar magnetic interactions (Hill et al., 2012; Pavlopoulos et al., 2016).

Another relevant family of MHNCs obtained by the selective heterogeneous nucleation/growth mechanism is represented by all-metallic anisotropic heterostructures. Tadpole-like MHNCs made of one-sided Au-tipped FePt nanorods, periodically Audecorated FePt nanorods and $\mathrm{Au}-\mathrm{FePt}$ necklace-like heterostructures were synthesized upon reduction of $\mathrm{Au}-\mathrm{OLAM}$ complex on preformed FePt nanorod seeds in organic media under controlled $\mathrm{Ar}$ or $\mathrm{Ar} / \mathrm{H}_{2}$ atmosphere at $25-80^{\circ} \mathrm{C}$ (Mao et al., 2011; $\mathrm{Wu}$ et al., 2011b). Under the specified reaction conditions, the regioselectivity for the possible destination sites of $\mathrm{Au}$ was found to correlate with the characteristics of the reducing atmosphere (Wu et al., 2011b) or with the reducibility of the starting Au molecular precursor (Mao et al., 2011), which indeed appeared to dictate the number of Au nucleation sites on the FePt seeds. To explain the formation of a periodic arrangements of $\mathrm{Au}$ domains over the thin, high aspect-ratio FePt nanorod seeds (Mao et al., 2011), an intraparticle Ostwarld ripening mechanism (Mokari et al., 2005) was invoked. All these FePt-Au MHNCs exhibited appealing electrocatalytic activity for $\mathrm{O}_{2}$ reduction (Mao et al., 2011), catalytic activity for methanol oxidation and better tolerance to poisoning $\mathrm{CO}$ on the catalyst surface compared to commercial Pt catalysts (Wu et al., 2011b).

In a very recent case study, selective growth at the apexes of anisotropic seeds was observed to result in shaped domains of the secondary material (Liakakos et al., 2014). Anisole solutions containing an organometallic iron precursor, $\mathrm{Fe}\left[\mathrm{N}-\left(\mathrm{SiMe}_{3}\right)_{2}\right]_{2}$, lauric acid (LA), and hexadecylamine (HDA) were reacted with HDA-capped Co nanorods at $150^{\circ} \mathrm{C}$ under $\mathrm{H}_{2}$ for $24 \mathrm{~h}$, yielding dumbbell-like MHNCs (Figure 7E) in which large cubic-shaped Fe domains with concave facets covered the termini of the starting Co seeds (Liakakos et al., 2014). The growth of Fe started from the Co nanorod tips and continued on the lateral facets along the longitudinal axis thereon, forming triangular prismatic domains. The latter eventually evolved into concave cubes surrounding the 
whole nanorod perimeter in proximity of both apexes. Some Co atoms appeared to be etched away from the seed tips during Fe overgrowth, whereas a small amount of non-crystallized $\mathrm{Fe}$ was found deposited on the Co domains. Interestingly, the soft iron nanocube domains behaved as easy nucleation centers that induced the magnetization reversal of the entire nanoheterostructure, leading to a drastic modification of the overall effective magnetic anisotropy (Liakakos et al., 2014).

Various prototypes of oxide-based MHNCs have been reported. A non-aqueous approach to magnetic-semiconductor $\gamma-\mathrm{Fe}_{2} \mathrm{O}_{3}-\mathrm{TiO}_{2}$ MHNCs (Figures 7I,J) with switchable binary to high-order topologies and tunable geometric parameters was developed (Buonsanti et al., 2009, 2010) The synthesis exploited brookite $\mathrm{TiO}_{2}$ nanorods with either rectangular- or tapered shape profiles (the latter terminating into arrow-like apexes with high-index facets) (Buonsanti et al., 2008) as substrate seeds for accommodating $\gamma-\mathrm{Fe}_{2} \mathrm{O}_{3}$ upon decomposition of $\mathrm{Fe}(\mathrm{CO})_{5}$ in OLAM/OLAC/hexadecan-1,2-diol mixtures at $280-300^{\circ} \mathrm{C}$. $\mathrm{The}^{\mathrm{TiO}} 2$ seeds exhibited size- and shape-dependent anisotropic reactivity, which allowed producing MHNCs individually made of a single $\mathrm{TiO}_{2}$ section supporting either one or multiple spherical $\gamma-\mathrm{Fe}_{2} \mathrm{O}_{3}$ domains at distinct locations in a highly controlled manner (Figure $\mathbf{7 A}$, paths 1 and 3). The $\mathrm{TiO}_{2} / \gamma-\mathrm{Fe}_{2} \mathrm{O}_{3}$ heteroepitaxy relationships were deciphered by CSLT-based analysis of HRTEM images of MHNCs viewed under different orientations (Randle, 1997; Markov, 2003; Narayan and Larson, 2003). In addition, the detailed spatial distribution of the component materials and of misfit-strain-induced lattice deformation (dilatation/compression, curvature, shear) across individual MHNCs were mapped by geometric phase analysis (GPA) of their HRTEM phase contrast (Hÿtch et al., 1998; Hytch and Plamann, 2001). These studies allowed understanding that the surface-interface energy balance associated with the formation of the different architectures could not be used as an unambiguous criterion to explain the observed regioselectivity, since the different MHNC configurations in fact appeared to be overall nearly energetically equivalent. Alternatively, the synthesis outcome was rationalized within the framework of a kinetic mechanistic picture assuming rapid establishment of a diffusion-limited growth regime for the foreign $\gamma-\mathrm{Fe}_{2} \mathrm{O}_{3}$ component. According to this model, $\gamma-\mathrm{Fe}_{2} \mathrm{O}_{3}$ overgrowth on the $\mathrm{TiO}_{2}$ seeds could switch from a thermodynamically controlled (corresponding to a non-selective $\gamma-\mathrm{Fe}_{2} \mathrm{O}_{3}$ deposition on the nanorod seeds) to a kinetically driven regime (corresponding to selective $\gamma-\mathrm{Fe}_{2} \mathrm{O}_{3}$ nucleation and growth on the seed apex) when highly anisotropically reactive seeds (the tapered nanorods with arrow-like terminations) were utilized under conditions (high precursor-to-seed ratios) favoring establishment of steep monomer gradients across the diffusion layer around each seed (Buonsanti et al., 2010). The as-synthesized $\gamma-\mathrm{Fe}_{2} \mathrm{O}_{3}-\mathrm{TiO}_{2}$ MHNCs displayed a rich scenario of photocatalytically driven charge retention/release properties and modified magnetic responses, which clearly diverged from those afforded by their individual components and physical-mixture counterparts (Buonsanti et al., 2009, 2010).

Intriguing branched $\alpha-\mathrm{Fe}_{2} \mathrm{O}_{3}-\mathrm{SnO}_{2} \mathrm{MHNCs}$ were built upon epitaxial $\mathrm{SnO}_{2}$ overgrowth on shaped $\alpha-\mathrm{Fe}_{2} \mathrm{O}_{3}$ seeds upon hydrothermal dehydratation of $\mathrm{Sn}(\mathrm{OH})_{6}^{2-}$ species (Niu et al., $2008,2010)$. For instance, when sixfold symmetric spindle-shaped $\alpha-\mathrm{Fe}_{2} \mathrm{O}_{3}$ seeds were used, multiple $\mathrm{SnO}_{2}$ domains indiscriminately nucleated onto each (110) facet, developing into small adjacent rods that progressively merged together laterally to decrease the overall surface energy. On the other hand, when overgrowth took place on cubic-shaped seeds, the secondary $\mathrm{SnO}_{2}$ nucleated on the $\alpha-\mathrm{Fe}_{2} \mathrm{O}_{3}$ facets slantwise at fixed angle, an arrangement that alleviated interfacial lattice mismatch and prevented the generation of misfit dislocations. A similar tendency toward branching was observed on employing hexahedron-shaped $\alpha-\mathrm{Fe}_{2} \mathrm{O}_{3}$ seeds (Niu et al., 2008). Compared to the bare $\alpha-\mathrm{Fe}_{2} \mathrm{O}_{3}$ particles, branched $\alpha-\mathrm{Fe}_{2} \mathrm{O}_{3}-\mathrm{SnO}_{2} \mathrm{MHNCs}$ showed prominent photocatalytic activity toward organic dye degradation under both visible and UV light irradiation (Niu et al., 2010). Worthy to mention are also hyperbranched $\alpha-\mathrm{Fe}_{2} \mathrm{O}_{3}-\mathrm{SnO}_{2}$ heterostructures with a sixfold symmetry, composed of a $\mathrm{SnO}_{2}$ nanowire stem and $\alpha-\mathrm{Fe}_{2} \mathrm{O}_{3}$ nanorod branches, which were generated by hydrothermal overgrowth of iron oxide on preformed $\mathrm{SnO}_{2}$ nanowire substrates prepared by a vapor-transport deposition route (Zhou et al., 2011). Such architectures demonstrated superior performance as anode material for lithium-ion batteries as a result of the synergistic effect of the $\alpha-\mathrm{Fe}_{2} \mathrm{O}_{3}$ and $\mathrm{SnO}_{2}$ components arranged in branched architectures.

\section{Surfactant-Controlled Regioselective Heterogeneous Nucleation and Growth}

For several material associations, careful investigation of the conditions underlying selection of MHNCs in non-equivalent topologies has suggested that the site-dependent accessibility and chemical reactivity of anisotropically shaped seeds may be governed by dynamic facet-preferential adhesion of surfactants or ligands (Figures 8A-C), a mechanism that has frequently been invoked to explain anisotropic growth of NCs (Burda et al., 2005; Cozzoli et al., 2006; Jun et al., 2006; Baghbanzadeh et al., 2011). Examples of elaborate MHNCs, which demonstrate the involvement of these pathways, are reported in Figures 8D-L.

A clear influence of the growth environment on the ultimate location of the secondary material domains was drawn in the synthesis of magnetic-plasmonic $\mathrm{Au}-\mathrm{Co}-\mathrm{Au}$ nanodumbbells (Figure 8D) accomplished by reacting Co nanorods with a $\mathrm{AuCl}$ (tetrahydrothiophene) complex precursor in the presence of LA and HAD (Wetz et al., 2007). In this system, the Co seeds triggered the otherwise kinetically hindered reduction of $\mathrm{Au}(\mathrm{I})$ to $\mathrm{Au}(0)$, while a dense protective coating of surface-adsorbed LA and HDA on the longitudinal sidewalls of the nanorods restricted Au deposition to their apexes only (Figure 8A).

Magnetic/semiconductor Co-functionalized anatase $\mathrm{TiO}_{2}$ nanorods (Figure 8E) were synthesized by manipulating the pyrolysis of $\mathrm{Co}_{2}(\mathrm{CO})_{8}$ under assistance of octanoic acid (OCAC) and OLAM at $250-280^{\circ} \mathrm{C}$ (Casavola et al., 2007). The OCAC and OLAM concentrations were judiciously regulated during the synthesis course as a means of modulating the accessibility of the reactive cobalt species to the $\mathrm{TiO}_{2}$ nanorod facets exposed at the apexes and at the longitudinal sidewalls. Such adjustment enabled switching heterogeneous Co nucleation from a regioselective to a non-selective decoration regime, 
in which multiple metal domains formed either on the tips only or indiscriminately over the whole body of the $\mathrm{TiO}_{2}$ seeds (Figure 8A). According to CSLT-based interpretation (Randle, 1997), both types of $\mathrm{TiO}_{2}$ HNCs were regarded as being nearly equivalent in terms of interfacial strain that had to be accommodated, regardless of the different structure types and densities of heterojunctions attained. Hence, the site-preferential Co overgrowth was rationalized as being a process that compensated for the increase in the overall surface energy caused by progressive depletion of the organic ligands on the seed surface at increasingly low surfactant content, to an extent that strongly depended on the type of facets involved in the depassivation process. As the two materials communicated through rather extended interfaces, the magnetic anisotropy of Co was found to be unpredictably altered due to the peculiar structural and/or electronic features attained at the heterojunctions with the non-magnetic $\mathrm{TiO}_{2}$ support (Casavola et al., 2007).

To achieve Co deposition on eccentric CdSe@CdS core@shell nanorods and $\gamma-\mathrm{Fe}_{2} \mathrm{O}_{3}$ tetrapods upon $\mathrm{Co}_{2}(\mathrm{CO})_{8}$ decomposition, non-coordinating ODE was selected as the reaction medium, without any extra surfactants or ligands added in (Casavola et al., 2009; Deka et al., 2009). The seeds became activated toward heterogeneous nucleation due to partial displacement of their original capping molecules A technique that involved controllably slow $\mathrm{Co}_{2}(\mathrm{CO})_{8}$ addition and delayed delivery of excess OLAC to a stage at which Co growth had almost reached completion, guaranteed the high-yield formation of heterostructures with high colloidal stability (Figures 8B,C). In the first documented case (Figure 8F), matchstick-like MHNCs, made of single-Co-tipped CdSe@CdS nanorods were obtained, whereby the Co domain formed on the apex that was diametrically opposed to the CdS region embedding the CdSe core (Deka et al., 2009). This outcome was consistent with the growth mechanism of CdSe@CdS core@shell nanorods themselves, which involved asymmetric unidirectional development of the CdS rodlike shell out of the CdSe seed cores (Carbone et al., 2007). Pertinent control syntheses, in which corresponding CdS-only nanorod were utilized as seeds, clarified that the inner core@shell arrangement of the CdSe@CdS core@shell seeds was an essential structural prerequisite to ensuring that the apexes acted as the exclusive nucleation sites. Thus, the unique seeding behavior was tentatively explained as being governed by electronic effects, in relation to the different inner dipole moment held by the CdSe@CdS seeds, with respect to their CdS-only counterparts. Interestingly, the CdSe@CdS-Co MHNCs exhibited appreciable luminescence in spite of photoexcited electrons transferring from the semiconductor to the metal domain and showed ferromagnetic-like behavior at room temperature, an anomaly that could arise from the unique structure of the intervening heterojunctions. The second case (Figures 8G-L) pertains MHNCs made of a $\gamma-\mathrm{Fe}_{2} \mathrm{O}_{3}$ tetrapod skeleton randomly decorated with multiple Co domains (Casavola et al., 2009). In these branched heterostructures ferrimagnetic-ferromagnetic exchange coupling resulted in a wealth of unique magnetic properties, including noticleable exchange bias, increased saturation magnetization and coercivity, and enhanced thermal stability.

Relying upon similar synthetic concepts, $\mathrm{MNHC}$ made of $\mathrm{ZnO}$ nanorod sections decorated with multiple Fe@Fe $\mathrm{O}_{y}$ core@shell nanospheres (Kostopoulou et al., 2012) were synthesized by performing heterogeneous nucleation of $\mathrm{Fe}$ on preformed $\mathrm{ZnO}$ nanorod seeds upon decomposing $\mathrm{Fe}(\mathrm{CO})_{5}$ in a non-coordinating ODE (i.e., in the absence of extra ligands or surfactants) at $230^{\circ} \mathrm{C}$, which was followed by partial surface air oxidation of the Fe domains to corresponding $\mathrm{Fe}_{\mathrm{F}} \mathrm{Fe}_{x} \mathrm{O}_{y}$ core@shell domains (Figure 8A). Interestingly, these MHNCs exhibited the band-edge $\mathrm{UV}$ photoluminescence of $\mathrm{ZnO}$ and size-dependent magnetic exchange-bias effects.

Finally, a more recent report documents the attainment of MHNCs with branched topology, composed of multiply Codecorated $\mathrm{CoO}$ tetrapods, through a one-pot protocol (Deng et al., 2014). Nucleation and growth of the CoO skeleton, and heterogeneous deposition of Co thereon, were found to be events controllably induced at different temperatures $\left(200^{\circ} \mathrm{C}\right.$ and $320^{\circ} \mathrm{C}$, respectively) during the thermal processing of cobalt acetylacetonate and 1,2-dodecanediol reducing agent in hot ODE.

\section{Strain-Driven Heterostructure Topology}

MHNCs with interesting structural features can be generated as a consequence of the emergence of intense strain fields during heteroepitaxial growth (Figure 9A). The cases reported in Figures 9B-E illustrate how strain could influence the growth and the topology of the colloidal heterostructures.

Asymmetric binary MHNCs, each made of one rod-shaped $\mathrm{TiO}_{2}$ section and one size-tunable $\gamma-\mathrm{Fe}_{2} \mathrm{O}_{3}$ spherical domain attached longitudinally (Figures 9B,C) were obtained by decomposing $\mathrm{Fe}(\mathrm{CO})_{5}$ on anatase $\mathrm{TiO}_{2}$ nanorod seeds in a ternary mixture of OLAC, OLAM and hexadecan-1, 2-diol at $240-300^{\circ} \mathrm{C}$ (Buonsanti et al., 2006). CSLT-based analysis of the heteroepitaxy relationships (Randle, 1997; Markov, 2003; Narayan and Larson, 2003) holding between the $\mathrm{TiO}_{2}$ and $\gamma-\mathrm{Fe}_{2} \mathrm{O}_{3}$, and study of GPA-derived maps (Hÿtch et al., 1998; Hytch and Plamann, 2001) of the spatial distribution of the component phases across individual HNCs shed light into some peculiar aspects of the growth mechanism of the MHNCs. The $\gamma-\mathrm{Fe}_{2} \mathrm{O}_{3}$ deposition was understood to proceed as a means of selectively eliminating highenergy edges on the longitudinal stepped sidewalls of the $\mathrm{TiO}_{2}$ seeds. However, the huge interfacial strain (8-11\%) restricted the degree of "wetting" of the groove facets underneath the edges, making the supporting $\mathrm{TiO}_{2}$ section deformed and curved toward the $\gamma-\mathrm{Fe}_{2} \mathrm{O}_{3}$ sphere, as evidenced, for example, by HAADF-STEM imaging (Figure 9C) and GPA-constructed phase maps. The $\mathrm{TiO}_{2}$ and $\gamma-\mathrm{Fe}_{2} \mathrm{O}_{3}$ lattices coincidently matched via a rather limited junction area at which the near-interface planes of the respective materials were locally bent. This curving allowed the interfacial strain to be relieved to a remarkable extent at a proportionally smaller cost of increased surface energy. This mechanism justifies the inhibition of reiterated deposition events and the modest size to which the $\gamma-\mathrm{Fe}_{2} \mathrm{O}_{3}$ domains could ultimately be grown. Due to the small contact area shared with $\mathrm{TiO}_{2}$, the magnetic properties of the MHNCs essentially resembled those of isolated $\gamma-\mathrm{Fe}_{2} \mathrm{O}_{3}$ particles (Buonsanti et al., 2006).

In another report, MHNCs with analogous topologies, made of a spinel $\mathrm{Fe}_{x} \mathrm{O}_{y}$ nanorod section asymmetrically decorated with a spherical $\mathrm{Au} @ \mathrm{Fe}_{x} \mathrm{O}_{y}$ core@shell domain (Figures 9D,E), were 
generated through an opposite reaction sequence (George et al., 2011b). Primary Au seeds were combined with $\mathrm{Fe}(\mathrm{CO})_{5}$ in ODEdiluted mixtures of OLAM, OLAC and dodecyldimethylammonium bromide (DDAB) at $300^{\circ} \mathrm{C}$. A thin, nearly ubiquitous, yet discontinuous shell of spinel $\mathrm{Fe}_{x} \mathrm{O}_{y}$ initially formed on the spherical Au seeds. However, upon prolonged annealing at high temperature, the combined effects of the strain increasing at the $\mathrm{Au} / \mathrm{Fe}_{x} \mathrm{O}_{y}$ interface and of the facet-selective binding of DDAB on $\mathrm{Fe}_{x} \mathrm{O}_{y}$ promoted breaking of the $\mathrm{Fe}_{x} \mathrm{O}_{y}$ growth symmetry, leading to tangential evolution of a $\mathrm{Fe}_{x} \mathrm{O}_{y}$ nanorod out of each $\mathrm{Au} @ \mathrm{Fe}_{x} \mathrm{O}_{y}$ core@shell intermediate. This mechanism was corroborated by topological and structural analyses of corresponding all- $\mathrm{Fe}_{x} \mathrm{O}_{y}$ nanostructures with mixed spherical and linear dimensionality, which were derived upon $\mathrm{I}_{2}$-driven leaching of $\mathrm{Au}$ from the $\mathrm{Au} @ \mathrm{Fe}_{x} \mathrm{O}_{y}-\mathrm{Fe}_{x} \mathrm{O}_{y}$ nanorod heterostructures (Figure 9E).

\section{CONCLUSION}

Design and construction of modular MHNCs that group epitaxially connected NC domains of magnetic and non-magnetic materials stand out as a frontier research area in which nanochemistry approaches have progressed tremendously over the past years. The challenges posed by the wet-chemical synthesis of colloidal "nanocrystal molecules" reflect the inherent difficulty of refining the ability to tailor specific nanoscale inorganic NC modules and of deepening the understanding of the intricately entangled thermodynamic conditions and kinetic processes that may allow their combination via programed heteroepitaxy in liquid media. Synthetic achievements and characterization reports documented so far suggest that an increased level of topological sophistication and regioselectivity in MHNC design and engineering should be heavily founded on far deeper mechanistic knowledge of the solid-state and liquid-phase formation pathways that underlie MHNC evolution under carefully specified conditions.

As of today, full realization of the technological potential of MHNCs is hindered by the level of synthetic precision and property control with which these nanoheterostructures can be engineered to meet specific purposes. Unfortunately, successful access to multifunctionality often conflicts with partial degradation of

\section{REFERENCES}

Amirav, L., and Alivisatos, A. P. (2010). Photocatalytic hydrogen production with tunable nanorod heterostructures. J. Phys. Chem. Lett. 1, 1051-1054. doi: $10.1021 /$ jz100075c

An, K., and Hyeon, T. (2009). Synthesis and biomedical applications of hollow nanostructures. Nano Today 4, 359-373. doi:10.1016/j.nantod. 2009.06.013

Armelles, G., Cebollada, A., Garcia-Martin, A., and Gonzalez, M. U. (2013). Magnetoplasmonics: combining magnetic and plasmonic functionalities. Adv. Opt. Mater. 1, 10-35. doi:10.1002/adom.201200011

Baghbanzadeh, M., Carbone, L., Cozzoli, P. D., and Kappe, C. O. (2011). Microwave-assisted synthesis of colloidal inorganic nanocrystals. Angew. Chem. Int. Ed. 50, 11312-11359. doi:10.1002/anie.201101274

Banin, U., Ben-Shahar, Y., and Vinokurov, K. (2014). Hybrid semiconductor-metal nanoparticles: from architecture to function. Chem. Mater. 26, 97-110. doi:10.1021/cm402131n some native properties of the concerned component modules due, for example, to unfavorable changes in electronic structure and/or to formation of defective interfaces. In many cases, it remains hard to unambiguously unveil and discriminate mere proximity effects from the genuine emergence of new or abnormal magnetic behavior or other chemical-physical properties as a consequence of the electronic interactions and exchange coupling via the relevant heterojunctions, especially for associations of magnetic and non-magnetic materials. The support of theoretical investigations, which are still rather sparse, would be of immense significance and usefulness in an effort toward decoupling the structure and roles played by nanoscale heterointerfaces (Xu et al., 2015; Mathew et al., 2016).

On the long term, it can be envisioned that development of an ingenious mechanism-driven design framework and synthetic capabilities for the elaboration of MHNCs will pave the way to both fundamental comprehension and practical utilization of novel unconventional properties and functionalities, particularly in the emerging fields of spintronics and magnetoplasmonics, green catalysis and biomedical theranostics.

\section{AUTHOR CONTRIBUTIONS}

PDC conceived and drafted the work; all the authors made substantial contribution to the design of the article, the acquisition, analysis, or interpretation of data for the work, and helped revising it critically for important intellectual content; they all approved the final version to be published; they agreed to be accountable for all aspects of the work in ensuring that questions related to the accuracy or integrity of any part of the work are appropriately investigated and resolved.

\section{FUNDING}

The authors acknowledge financial support from the Apulia Regional Government through Project "NANOAPULIA" (proj. cod. MDI6SR1 - CUP B38C14001140008) and from the Fondazione Cariplo through Project "Chemical synthesis and characterization of magneto-plasmonic nano-heterostructures" (proj. no. 2010-0612).

Bigall, N. C., Parak, W. J., and Dorfs, D. (2012). Fluorescent, magnetic and plasmonic - hybrid multifunctional colloidal nano objects. Nano Today 7, 282-296. doi:10.1016/j.nantod.2012.06.007

Bouet, C., Tessier, M. D., Ithurria, S., Mahler, B., Nadal, B., and Dubertret, B. (2013). Flat colloidal semiconductor nanoplatelets. Chem. Mater. 25, 1262-1271. doi:10.1021/cm303786a

Bradley, M. J., Biacchi, A. J., and Schaak, R. E. (2013). Chemical transformation of $\mathrm{Pt}-\mathrm{Fe}_{3} \mathrm{O}_{4}$ colloidal hybrid nanoparticles into $\mathrm{PtPb}-\mathrm{Fe}_{3} \mathrm{O}_{4}$ and $\mathrm{Pt}_{3} \mathrm{Sn}-\mathrm{Fe}_{3} \mathrm{O}_{4}$ heterodimers and $\left(\mathrm{PtPb}-\mathrm{Fe}_{3} \mathrm{O}_{4}\right)_{\mathrm{n}}$ nanoflowers. Chem. Mater. 25, 1886-1892. doi: $10.1021 / \mathrm{cm} 4005163$

Bradley, M. J., Read, C. G., and Schaak, R. E. (2015). Pt-Au nanoparticle heterodimers as seeds for $\mathrm{Pt}-\mathrm{Au}$-metal sulfide heterotrimers: thermal stability and chemoselective growth characteristics. J. Phys. Chem. C 119, 8952-8959. doi:10.1021/acs.jpcc.5b01274

Buck, M. R., Bondi, J. F., and Schaak, R. E. (2012). A total-synthesis framework for the construction of high-order colloidal hybrid nanoparticles. Nat. Chem. 4, 37-44. doi:10.1038/nchem.1195 
Buck, M. R., and Schaak, R. E. (2013). Emerging strategies for the total synthesis of inorganic nanostructures. Angew. Chem. Int. Ed. 52, 6154-6178. doi:10.1002/ anie. 201207240

Buonsanti, R., Casavola, M., Caputo, G., and Cozzoli, P. D. (2007). Advances in the chemical fabrication of complex multimaterial nanocrystals. Recent Pat. Nanotechnol. 1, 224-232. doi:10.2174/187221007782360420

Buonsanti, R., Grillo, V., Carlino, E., Giannini, C., Curri, M. L., Innocenti, C., et al. (2006). Seeded growth of asymmetric binary nanocrystals made of a semiconductor $\mathrm{TiO}_{2}$ rodlike section and a magnetic $\gamma-\mathrm{Fe}_{2} \mathrm{O}_{3}$ spherical domain. J. Am. Chem. Soc. 128, 16953-16970. doi:10.1021/ja066557h

Buonsanti, R., Grillo, V., Carlino, E., Giannini, C., Gozzo, F., Garcia-Hernandez, M., et al. (2010). Architectural control of seeded-grown magnetic-semicondutor iron oxide- $\mathrm{TiO}_{2}$ nanorod heterostructures: the role of seeds in topology selection. J. Am. Chem. Soc. 132, 2437-2464. doi:10.1021/ja910322a

Buonsanti, R., Grillo, V., Carlino, E., Giannini, C., Kipp, T., Cingolani, R., et al. (2008). Nonhydrolytic synthesis of high-quality anisotropically shaped brookite $\mathrm{TiO}_{2}$ nanocrystals. J. Am. Chem. Soc. 130, 11223-11233. doi:10.1021/ja803559b

Buonsanti, R., Snoeck, E., Giannini, C., Gozzo, F., Garcia-Hernandez, M., Garcia, M. A., et al. (2009). Colloidal semiconductor/magnetic heterostructures based on iron-oxide-functionalized brookite $\mathrm{TiO}_{2}$ nanorods. Phys. Chem. Chem. Phys. 11, 3680-3691. doi:10.1039/b821964h

Burda, C., Chen, X. B., Narayanan, R., and El-Sayed, M. A. (2005). Chemistry and properties of nanocrystals of different shapes. Chem. Rev. 105, 1025-1102. doi:10.1021/cr030063a

Carbone, L., and Cozzoli, P. D. (2010). Colloidal heterostructured nanocrystals: synthesis and growth mechanisms. Nano Today 5, 449-493. doi:10.1016/j. nantod.2010.08.006

Carbone, L., Nobile, C., Giorgi, M. D., Sala, F. D., Morello, G., Pompa, P., et al. (2007). Synthesis and micrometer-scale assembly of colloidal CdSe/CdS nanorods prepared by a seeded growth approach. Nano Lett. 7, 2942-2950. doi:10.1021/nl0717661

Carenco, S., Moldovan, S., Roiban, L., Florea, I., Portehault, D., Valle, K., et al. (2016). The core contribution of transmission electron microscopy to functional nanomaterials engineering. Nanoscale 8, 1260-1279. doi:10.1039/c5nr05460e

Carey, G. H., Abdelhady, A. L., Ning, Z., Thon, S. M., Bakr, O. M., and Sargent, E. H. (2015). Colloidal quantum dot solar cells. Chem. Rev. 115, 12732-12763. doi:10.1021/acs.chemrev.5b00063

Casavola, M., Buonsanti, R., Caputo, G., and Cozzoli, P. D. (2008). Colloidal strategies for preparing oxide-based hybrid nanocrystals. Eur. J. Inorg. Chem. 837-854. doi:10.1002/ejic.200701047

Casavola, M., Falqui, A., Garcia, M. A., Garcia-Hernandez, M., Giannini, C., Cingolani, R., et al. (2009). Exchange-coupled bimagnetic cobalt/iron oxide branched nanocrystal heterostructures. Nano Lett. 9, 366-376. doi:10.1021/ nl803151n

Casavola, M., Grillo, V., Carlino, E., Giannini, C., Gozzo, F., Fernandez Pinel, E., et al. (2007). Topologically controlled growth of magnetic-metalfunctionalized semiconductor oxide nanorods. Nano Lett. 7, 1386-1395. doi:10.1021/nl070550w

Chatterjee, K., Sarkar, S., Jagajjanani Rao, K., and Paria, S. (2014). Core/shell nanoparticles in biomedical applications. Adv. Colloid Interface Sci. 209, 8-39. doi:10.1016/j.cis.2013.12.008

Chen, S., Si, R., Taylor, E., Janzen, J., and Chen, J. (2012). Synthesis of $\mathrm{Pd} / \mathrm{Fe}_{3} \mathrm{O}_{4}$ hybrid nanocatalysts with controllable interface and enhanced catalytic activities for CO oxidation. J. Phys. Chem. C 116, 12969-12976. doi:10.1021/jp3036204

Chen, X. B., Lou, Y. B., Samia, A. C., and Burda, C. (2003). Coherency strain effects on the optical response of core/shell heteronanostructures. Nano Lett. 3, 799-803. doi:10.1021/nl034243b

Chng, L. L., Erathodiyil, N., and Ying, J. Y. (2013). Nanostructured catalysts for organic transformations. Acc. Chem. Res. 46, 1825-1837. doi:10.1021/ar300197s

Choi, J. S., Jun, Y. W., Yeon, S. I., Kim, H. C., Shin, J. S., and Cheon, J. (2006). Biocompatible heterostructured nanoparticles for multimodal biological detection. J. Am. Chem. Soc. 128, 15982-15983. doi:10.1021/ja066547g

Choi, S.-H., Na, H. B., Park, Y. I., An, K., Kwon, S. G., Jang, Y., et al. (2008). Simple and generalized synthesis of oxide-metal heterostructured nanoparticles and their applications in multimodal biomedical probes. J. Am. Chem. Soc. 130, 15573-15580. doi:10.1021/ja805311x

Comin, A., Korobchevskaya, K., George, C., Diaspro, A., and Manna, L. (2012). Plasmon bleaching dynamics in colloidal gold-iron oxide nanocrystal heterodimers. Nano Lett. 12, 921-926. doi:10.1021/nl2039875
Costi, R., Saunders, A. E., and Banin, U. (2010). Colloidal hybrid nanostructures: a new type of functional materials. Angew. Chem. Int. Ed. 49, 4878-4897. doi:10.1002/anie. 200906010

Costi, R., Saunders, A. E., Elmalem, E., Salant, A., and Banin, U. (2008). Visible light-induced charge retention and photocatalysis with hybrid $\mathrm{CdSe}-\mathrm{Au}$ nanodumbbells. Nano Lett. 8, 637-641. doi:10.1021/nl0730514

Cozzoli, P. D., Pellegrino, T., and Manna, L. (2006). Synthesis, properties and perspectives of hybrid nanocrystal structures. Chem. Soc. Rev. 35, 1195-1208. doi:10.1039/b517790c

Cozzoli, P. D. (ed.). (2008). Advanced Wet-Chemical Synthetic Approaches to Inorganic Nanostructures. Kerala, India: Transworld Research Network.

de Mello Donegà, C. (2011). Synthesis and properties of colloidal heteronanocrystals. Chem. Soc. Rev. 40, 1512-1546. doi:10.1039/c0cs00055h

de Mello Donegà, C., Liljeroth, P., and Vanmaekelbergh, D. (2005). Physicochemical evaluation of the hot-injection method, a synthesis route for monodisperse nanocrystals. Small 1, 1152-1162. doi:10.1002/smll.200500239

De Trizio, L., and Manna, L. (2016). Forging colloidal nanostructures via cation exchange reactions. Chem. Rev. 116, 10852-10887. doi:10.1021/acs.chemrev. 5 b00739

Deka, S., Falqui, A., Bertoni, G., Sangregorio, C., Poneti, G., Morello, G., et al. (2009). Fluorescent asymmetrically cobalt-tipped CdSe@CdS core@shell nanorod heterostructures exhibiting room-temperature ferromagnetic behavior. J. Am. Chem. Soc. 131, 12817-12828. doi:10.1021/ja904493c

Deng, X., Yang, D., Tan, G., Li, X., Zhang, J., Liu, Q., et al. (2014). Bimagnetic h-Co/h-CoO nanotetrapods: preparation, nanoscale characterization, three-dimensional architecture and their magnetic properties. Nanoscale 6, 13710-13718. doi:10.1039/c4nr02287d

Dinsmore, A. D., Hsu, M. F., Nikolaides, M. G., Marquez, M., Bausch, A. R., and Weitz, D. A. (2002). Colloidosomes: selectively permeable capsules composed of colloidal particles. Science 298, 1006-1009. doi:10.1126/science.1074868

Duguet, E., Desert, A., Perro, A., and Ravaine, S. (2011). Design and elaboration of colloidal molecules: an overview. Chem. Soc. Rev. 40, 941-960. doi:10.1039/ C0CS00048E

Dunstan, D. J. (1997). Strain and strain relaxation in semiconductors. J. Mater. Sci. Mater. Electron. 8, 337-375. doi:10.1023/a:1018547625106

Elmalem, E., Saunders, A. E., Costi, R., Salant, A., and Banin, U. (2008). Growth of photocatalytic CdSe-Pt nanorods and nanonets. Adv. Mater. 20, 4312-4317. doi:10.1002/adma.200800044

Erdemir, D., Lee, A. Y., and Myerson, A. S. (2009). Nucleation of crystals from solution: classical and two-step models. Acc. Chem. Res. 42, 621-629. doi: $10.1021 / \operatorname{ar} 800217 \mathrm{x}$

Feyen, M., Weidenthaler, C., Schüth, F., and Lu, A.-H. (2010). Regioselectively controlled synthesis of colloidal mushroom nanostructures and their hollow derivatives. J. Am. Chem. Soc. 132, 6791-6799. doi:10.1021/ja101270r

Figuerola, A., Fiore, A., Di Corato, R., Falqui, A., Giannini, C., Micotti, E., et al. (2008). One-pot synthesis and characterization of size-controlled bimagnetic FePt-iron oxide heterodimer nanocrystals. J. Am. Chem. Soc. 130, 1477-1487. doi:10.1021/ja078034v

Franchini, I. R., Bertoni, G., Falqui, A., Giannini, C., Wang, L. W., and Manna, L. (2010). Colloidal PbTe-Au nanocrystal heterostructures. J. Mater. Chem. 20, 1357-1366. doi:10.1039/b915687a

Freeman, R., and Willner, I. (2012). Optical molecular sensing with semiconductor quantum dots (QDs). Chem. Soc. Rev. 41, 4067-4085. doi:10.1039/c2cs15357b

Frey, N. A., Peng, S., Cheng, K., and Sun, S. (2009). Magnetic nanoparticles: synthesis, functionalization, and applications in bioimaging and magnetic energy storage. Chem. Soc. Rev. 38, 2532-2542. doi:10.1039/b815548h

Gao, J., Gu, H., and Xu, B. (2009). Multifunctional magnetic nanoparticles: design, synthesis, and biomedical applications. Acc. Chem. Res. 42, 1097-1107. doi:10.1021/ar9000026

Gao, N., and Fang, X. (2015). Synthesis and development of graphene-inorganic semiconductor nanocomposites. Chem. Rev. 115, 8294-8343. doi:10.1021/ cr400607y

Gebauer, D., and Colfen, H. (2011). Prenucleation clusters and non-classical nucleation. Nano Today 6, 564-584. doi:10.1016/j.nantod.2011.10.005

Gentili, D., Foschi, G., Valle, F., Cavallini, M., and Biscarini, F. (2012). Applications of dewetting in micro and nanotechnology. Chem. Soc. Rev. 41, 4430-4443. doi:10.1039/c2cs35040h

George, C., Dorfs, D., Bertoni, G., Falqui, A., Genovese, A., Pellegrino, T., et al. (2011a). A cast-mold approach to iron oxide and Pt/iron oxide nanocontainers 
and nanoparticles with a reactive concave surface. J. Am. Chem. Soc. 133, 2205-2217. doi:10.1021/ja108781w

George, C., Genovese, A., Qiao, F., Korobchevskaya, K., Comin, A., Falqui, A., et al. (2011b). Optical and electrical properties of colloidal (spherical Au)(spinel ferrite nanorod) heterostructures. Nanoscale 3, 4647-4654. doi:10.1039/ clnr10768b

George, C., Genovese, A., Casu, A., Prato, M., Povia, M., Manna, L., et al. (2013). CO oxidation on colloidal $\mathrm{Au}_{0.80} \mathrm{Pd}_{0.20}-\mathrm{Fe}_{\mathrm{x}} \mathrm{O}_{\mathrm{y}}$ dumbbell nanocrystals. Nano Lett. 13, 752-757. doi:10.1021/nl304448p

Ghigna, P., and Spinolo, G. (2015). High-energy X-ray absorption spectroscopy in materials chemistry. Sci. Adv. Mater. 7, 2216-2233. doi:10.1166/sam.2015.2270

Ghosh Chaudhuri, R., and Paria, S. (2011). Core/shell nanoparticles: classes, properties, synthesis mechanisms, characterization, and applications. Chem. Rev. 112, 2373-2433. doi:10.1021/cr100449n

Giannini, C., Ladisa, M., Altamura, D., Siliqi, D., Sibillano, T., and De Caro, L. (2016). X-ray diffraction: a powerful technique for the multiple-length-scale structural analysis of nanomaterials. Crystals 6, 87. doi:10.3390/cryst6080087

Gu, H. W., Yang, Z. M., Gao, J. H., Chang, C. K., and Xu, B. (2005). Heterodimers of nanoparticles: formation at a liquid-liquid interface and particle-specific surface modification by functional molecules. J. Am. Chem. Soc. 127, 34-35. doi:10.1021/ja045220h

Gu, H. W., Zheng, R. K., Zhang, X. X., and Xu, B. (2004). Facile one-pot synthesis of bifunctional heterodimers of nanoparticles: a conjugate of quantum dot and magnetic nanoparticles. J. Am. Chem. Soc. 126, 5664-5665. doi:10.1021/ ja0496423

Habas, S. E., Yang, P., and Mokari, T. (2008). Selective growth of metal and binary metal tips on CdS nanorods. J. Am. Chem. Soc. 130, 3294-3295. doi:10.1021/ ja800104w

He, J., Liu, Y. J., Hood, T. C., Zhang, P., Gong, J. L., and Nie, Z. H. (2013). Asymmetric organic/metal(oxide) hybrid nanoparticles: synthesis and applications. Nanoscale 5, 5151-5166. doi:10.1039/c3nr34014g

He, S. L., Zhang, H. W., Delikanli, S., Qin, Y. L., Swihart, M. T., and Zeng, H. (2009). Bifunctional magneto-optical FePt-CdS hybrid nanoparticles. J. Phys. Chem. C 113, 87-90. doi:10.1021/jp806247f

Hill, L. J., Bull, M. M., Sung, Y., Simmonds, A. G., Dirlam, P. T., Richey, N. E., et al. (2012). Directing the deposition of ferromagnetic cobalt onto Pttipped CdSe@CdS nanorods: synthetic and mechanistic insights. ACS Nano 6, 8632-8645. doi:10.1021/nn3019859

Hodges, J. M., Biacchi, A. J., and Schaak, R. E. (2014). Ternary hybrid nanoparticle isomers: directing the nucleation of $\mathrm{Ag}$ on $\mathrm{Pt}-\mathrm{Fe}_{3} \mathrm{O}_{4}$ using a Solid-State Protecting Group. ACS Nano 8, 1047-1055. doi:10.1021/nn405943z

Hodges, J. M., Morse, J. R., Fenton, J. L., Ackerman, J. D., Alameda, L. T., and Schaak, R. E. (2016). Insights into the seeded-growth synthesis of colloidal hybrid nanoparticles. Chem. Mater. doi:10.1021/acs.chemmater.6b02795

Hodges, J. M., Morse, J. R., Williams, M. E., and Schaak, R. E. (2015). Microscopic investigation of chemoselectivity in $\mathrm{Ag}-\mathrm{Pt}-\mathrm{Fe}_{3} \mathrm{O}_{4}$ heterotrimer formation: mechanistic insights and implications for controlling high-order hybrid nanoparticle morphology. J. Am. Chem. Soc. 137, 15493-15500. doi:10.1021/ jacs.5b10254

Hou, X., Zhang, X., Chen, S., Kang, H., and Tan, W. (2012). Facile synthesis of $\mathrm{Ni} / \mathrm{Au}, \mathrm{Ni} / \mathrm{Ag}$ hybrid magnetic nanoparticles: new active substrates for surface enhanced Raman scattering. Colloids Surf. A Physicochem. Eng. Asp. 403, 148-154. doi:10.1016/j.colsurfa.2012.04.005

$\mathrm{Hu}, \mathrm{L}$., Zhang, R., and Chen, Q. (2014). Synthesis and assembly of nanomaterials under magnetic fields. Nanoscale 6, 14064-14105. doi:10.1039/c4nr05108d

$\mathrm{Hu}, \mathrm{S}$., and Wang, X. (2013). Ultrathin nanostructures: smaller size with new phenomena. Chem. Soc. Rev. 42, 5577-5594. doi:10.1039/c3cs00006k

Huang, J. M., Sun, Y. H., Huang, S. S., Yu, K., Zhao, Q., Peng, F., et al. (2011). Crystal engineering and SERS properties of $\mathrm{Ag}-\mathrm{Fe}_{3} \mathrm{O}_{4}$ nanohybrids: from heterodimer to core-shell nanostructures. J. Mater. Chem. 21, 17930-17937. doi:10.1039/ cljm13045e

Hytch, M. J., and Plamann, T. (2001). Imaging conditions for reliable measurement of displacement and strain in high-resolution electron microscopy. Ultramicroscopy 87, 199-212. doi:10.1016/S0304-3991(00)00099-1

Hÿtch, M. J., Snoeck, E., and Kilaas, R. (1998). Quantitative measurement of displacement and strain fields from HREM micrographs. Ultramicroscopy 74, 131-146. doi:10.1016/S0304-3991(98)00035-7

Jang, Y., Chung, J., Kim, S., Jun, S. W., Kim, B. H., Lee, D. W., et al. (2011a). Simple synthesis of $\mathrm{Pd}-\mathrm{Fe}_{3} \mathrm{O}_{4}$ heterodimer nanocrystals and their application as a magnetically recyclable catalyst for Suzuki cross-coupling reactions. Phys. Chem. Chem. Phys. 13, 2512-2516. doi:10.1039/c0cp01680b

Jang, Y., Kim, S., Jun, S. W., Kim, B. H., Hwang, S., Song, I. K., et al. (2011b) Simple one-pot synthesis of $\mathrm{Rh}-\mathrm{Fe}_{3} \mathrm{O}_{4}$ heterodimer nanocrystals and their applications to a magnetically recyclable catalyst for efficient and selective reduction of nitroarenes and alkenes. Chem. Commun. 47, 3601-3603. doi:10.1039/c0cc04816j

Jen-La Plante, I., Habas, S. E., Yuhas, B. D., Gargas, D. J., and Mokari, T. (2009). Interfacing metal nanoparticles with semiconductor nanowires. Chem. Mater. 21, 3662-3667. doi:10.1021/cm900775w

Jerison, E. R., Xu, Y., Wilen, L. A., and Dufresne, E. R. (2011). Deformation of an elastic substrate by a three-phase contact line. Phys. Rev. Lett. 106, 186103. doi:10.1103/PhysRevLett.106.186103

Jiang, G., Huang, Y., Zhang, S., Zhu, H., Wu, Z., and Sun, S. (2016). Controlled synthesis of $\mathrm{Au}-\mathrm{Fe}$ heterodimer nanoparticles and their conversion into $\mathrm{Au}$ $\mathrm{Fe}_{3} \mathrm{O}_{4}$ heterostructured nanoparticles. Nanoscale 8, 17947-17952. doi:10.1039/ c6nr06395k

Jiang, J., Gu, H., Shao, H., Devlin, E., Papaefthymiou, G. C., and Ying, J. Y. (2008). Bifunctional $\mathrm{Fe}_{3} \mathrm{O}_{4}-\mathrm{Ag}$ heterodimer nanoparticles for two-photon fluorescence imaging and magnetic manipulation. Adv. Mater. 20, 4403-4407. doi:10.1002/ adma.200800498

Jun, Y.-W., Choi, J.-S., and Cheon, J. (2006). Shape control of semiconductor and metal oxide nanocrystals through nonhydrolytic colloidal routes. Angew. Chem. Int. Ed. 45, 3414-3439. doi:10.1002/anie.200503821

Jun, Y.-W., Choi, J.-S., and Cheon, J. (2007). Heterostructured magnetic nanoparticles: their versatility and high performance capabilities. Chem. Commun. 1203-1214. doi:10.1039/b614735f

Kaewsaneha, C., Tangboriboonrat, P., Polpanich, D., Eissa, M., and Elaissari, A. (2013). Janus colloidal particles: preparation, properties, and biomedical applications. ACS Appl. Mater. Interfaces 5, 1857-1869. doi:10.1021/am302528g

Kamat, P. V. (2007). Meeting the clean energy demand: nanostructure architectures for solar energy conversion. J. Phys. Chem. C 111, 2834-2860. doi:10.1021/ jp066952u

Kim, B. H., Hackett, M. J., Park, J., and Hyeon, T. (2013). Synthesis, characterization, and application of ultrasmall nanoparticles. Chem. Mater. 26, 59-71. doi: $10.1021 / \mathrm{cm} 402225 \mathrm{z}$

Kim, M., and Song, H. (2014). Precise adjustment of structural anisotropy and crystallinity on metal- $\mathrm{Fe}_{3} \mathrm{O}_{4}$ hybrid nanoparticles and its influence on magnetic and catalytic properties. J. Mater. Chem. C 2, 4997-5004. doi:10.1039/c4tc00416g

Koo, B., and Korgel, B. A. (2008). Coalescence and interface diffusion in linear CdTe/CdSe/CdTe heterojunction nanorods. Nano Lett. 8, 2490-2496. doi:10.1021/nl8015126

Korobchevskaya, K., George, C., Diaspro, A., Manna, L., Cingolani, R., and Comin, A. (2011). Ultrafast carrier dynamics in gold/iron-oxide nanocrystal heterodimers. Appl. Phys. Lett. 99, 011907. doi:10.1063/1.3609324

Kostopoulou, A., Thétiot, F., Tsiaoussis, I., Androulidaki, M., Cozzoli, P. D., and Lappas, A. (2012). Colloidal anisotropic $\mathrm{ZnO}-\mathrm{Fe} @ \mathrm{Fe}_{\mathrm{x}} \mathrm{O}_{\mathrm{y}}$ nanoarchitectures with interface-mediated exchange-bias and band-edge ultraviolet fluorescence. Chem. Mater. 24, 2722-2732. doi:10.1021/cm3008182

Krylova, G., Giovanetti, L. J., Requejo, F. G., Dimitrijevic, N. M., Prakapenka, A., and Shevchenko, E. V. (2012). Study of nucleation and growth mechanism of the metallic nanodumbbells. J. Am. Chem. Soc. 134, 4384-4392. doi:10.1021/ ja211459p

Kwon, K.-W., Lee, B. H., and Shim, M. (2006). Structural evolution in metal oxide/semiconductor colloidal nanocrystal heterostructures. Chem. Mater. 18, 6357-6363. doi:10.1021/cm0621390

Kwon, K. W., and Shim, M. (2005). $\gamma-\mathrm{Fe}_{2} \mathrm{O}_{3} / \mathrm{II}-\mathrm{VI}$ sulfide nanocrystal heterojunctions. J. Am. Chem. Soc. 127, 10269-10275. doi:10.1021/ja051713q

Kwon, S. G., and Hyeon, T. (2008). Colloidal chemical synthesis and formation kinetics of uniformly sized nanocrystals of metals, oxides, and chalcogenides. Acc. Chem. Res. 41, 1696-1709. doi:10.1021/ar8000537

Kwon, S. G., Krylova, G., Phillips, P. J., Klie, R. F., Chattopadhyay, S., Shibata, T., et al. (2015). Heterogeneous nucleation and shape transformation of multicomponent metallic nanostructures. Nat. Mater. 14, 215-223. doi:10.1038/ nmat4115

Lattuada, M., and Hatton, T. A. (2011). Synthesis, properties and applications of Janus nanoparticles. Nano Today 6, 286-308. doi:10.1016/j.nantod.2011.04.008

Lee, J.-S., Bodnarchuk, M. I., Shevchenko, E. V., and Talapin, D. V. (2010a). "Magnet-in-the-semiconductor" FePt-PbS and FePt-PbSe nanostructures: 
magnetic properties, charge transport, and magnetoresistance. J. Am. Chem. Soc. 132, 6382-6391. doi:10.1021/ja100029s

Lee, Y., Garcia, M. A., Frey Huls, N. A., and Sun, S. (2010b). Synthetic tuning of the catalytic properties of $\mathrm{Au}-\mathrm{Fe}_{3} \mathrm{O}_{4}$ nanoparticles. Angew. Chem. Int. Ed. 49, 1271-1274. doi:10.1002/anie.200906130

Lee, K. S., Anisur, R. M., Kim, K. W., Kim, W. S., Park, T. J., Kang, E. J., et al. (2012). Seed size-dependent formation of $\mathrm{Fe}_{3} \mathrm{O}_{4} / \mathrm{MnO}$ hybrid nanocrystals: selective, magnetically recyclable catalyst systems. Chem. Mater. 24, 682-687. doi:10.1021/cm2027724

Lee, K. T., and Cho, J. (2011). Roles of nanosize in lithium reactive nanomaterials for lithium ion batteries. Nano Today 6, 28-41. doi:10.1016/j.nantod.2010.11.002

Leung, K. C.-F., Xuan, S., Zhu, X., Wang, D., Chak, C.-P., Lee, S.-F., et al. (2012). Gold and iron oxide hybrid nanocomposite materials. Chem. Soc. Rev. 41, 1911-1928. doi: $10.1039 / \mathrm{C} 1 \mathrm{CS} 15213 \mathrm{~K}$

Levin, C. S., Hofmann, C., Ali, T. A., Kelly, A. T., Morosan, E., Nordlander, P., et al. (2009). Magnetic-plasmonic core-shell nanoparticles. ACS Nano 3, 1379-1388. doi:10.1021/nn900118a

Lhuillier, E., Pedetti, S., Ithurria, S., Nadal, B., Heuclin, H., and Dubertret, B. (2015). Two-dimensional colloidal metal chalcogenides semiconductors: synthesis, spectroscopy, and applications. Acc. Chem. Res. 48, 22-30. doi:10.1021/ ar500326c

Li, H. B., Kanaras, A. G., and Manna, L. (2013). Colloidal branched semiconductor nanocrystals: state of the art and perspectives. Acc. Chem. Res. 46, 1387-1396. doi:10.1021/ar3002409

Li, T., Senesi, A. J., and Lee, B. (2016). Small angle X-ray scattering for nanoparticle research. Chem. Rev. 116, 11128-11180. doi:10.1021/acs.chemrev.5b00690

Li, X. L., Wang, C. X., and Yang, G. W. (2014). Thermodynamic theory of growth of nanostructures. Progr. Mater. Sci. 64, 121-199. doi:10.1016/j.pmatsci.2014.03. 002

Li, Y., Zhang, Q., Nurmikko, A. V., and Sun, S. (2005). Enhanced magnetooptical response in dumbbell-like $\mathrm{Ag}-\mathrm{CoFe}_{2} \mathrm{O}_{4}$ nanoparticle pairs. Nano Lett. 5, 1689-1692. doi:10.1021/nl050814j

Liakakos, N., Gatel, C., Blon, T., Altantzis, T., Lentijo-Mozo, S., Garcia-Marcelot, C., et al. (2014). Co-Fe nanodumbbells: synthesis, structure, and magnetic properties. Nano Lett. 14, 2747-2754. doi:10.1021/nl500734k

Liang, W. I., Zhang, X. W., Zan, Y. L., Pan, M., Czarnik, C., Bustillo, K., et al. (2015). In situ study of $\mathrm{Fe}_{3} \mathrm{Pt}_{-}-\mathrm{Fe}_{2} \mathrm{O}_{3}$ core-shell nanoparticle formation. J. Am. Chem. Soc. 137, 14850-14853. doi:10.1021/jacs.5610076

Liao, F., Lo, B. T. W., and Tsang, E. (2016). The applications of nano-heterojunction in optical and thermal catalysis. Eur. J. Inorg. Chem. 2016, 1924-1938. doi:10.1002/ejic.201501213

Lim, J., and Majetich, S. A. (2013). Composite magnetic-plasmonic nanoparticles for biomedicine: manipulation and imaging. Nano Today 8, 98-113. doi:10.1016/ j.nantod.2012.12.010

Lin, F. H., and Doong, R. A. (2011). Bifunctional $\mathrm{Au}-\mathrm{Fe}_{3} \mathrm{O}_{4}$ heterostructures for magnetically recyclable catalysis of nitrophenol reduction. J. Phys. Chem. C 115, 6591-6598. doi:10.1021/jp110956k

Liu, F., Zhu, J., Yang, W., Dong, Y., Hou, Y., Zhang, C., et al. (2014). Building nanocomposite magnets by coating a hard magnetic core with a soft magnetic shell. Angew. Chem. Int. Ed. 53, 2176-2180. doi:10.1002/anie.201309723

Liu, J., Qiao, S. Z., Chen, J. S., Lou, X. W., Xing, X. R., and Lu, G. Q. (2011). Yolk/shell nanoparticles: new platforms for nanoreactors, drug delivery and lithium-ion batteries. Chem. Commun. 47, 12578-12591. doi:10.1039/c1cc13658e

Liu, S., Guo, S., Sun, S., and You, X.-Z. (2015). Dumbbell-like Au- $\mathrm{Fe}_{3} \mathrm{O}_{4}$ nanoparticles: a new nanostructure for supercapacitors. Nanoscale 7, 4890-4893. doi:10.1039/c5nr00135h

Liu, Y. L., and Walker, A. R. H. (2010). Facile one-pot synthesis of metalsemiconductor hybrid nanocrystals via chemical transformation: the case of $\mathrm{Cu}-\mathrm{Cu}_{\mathrm{x}} \mathrm{S}$ heterodimers and hetero-oligomers. J. Phys. Chem. C 114, 4264-4271. doi:10.1021/jp908981n

López-Ortega, A., Estrader, M., Salazar-Alvarez, G., Roca, A. G., and Nogués, J. (2015). Applications of exchange coupled bi-magnetic hard/soft and soft/hard magnetic core/shell nanoparticles. Phys. Rep. 553, 1-32. doi:10.1016/j.physrep. 2014.09.007

Mao, J., Cao, X., Zhen, J., Shao, H., Gu, H., Lu, J., et al. (2011). Facile synthesis of hybrid nanostructures from nanoparticles, nanorods and nanowires. J. Mater. Chem. 21, 11478-11481. doi:10.1039/c1jm10349k

Mao, Y. W., Yi, P. W., Deng, Z. W., and Ge, J. P. (2013). $\mathrm{Fe}_{3} \mathrm{O}_{4}-\mathrm{Ag}$ heterostructure nanocrystals with tunable Ag domains and magnetic properties. CrystEngComm 15, 3575-3581. doi:10.1039/c3ce40095f
Markov, I. V. (2003). Crystal Growth for Beginners: Fundamentals of Nucleation, Crystal Growth, and Epitaxy. Singapore: World Scientific.

Mathew, K., Singh, A. K., Gabriel, J. J., Choudhary, K., Sinnott, S. B., Davydov, A. V., et al. (2016). MPInterfaces: a materials project based python tool for highthroughput computational screening of interfacial systems. Comput. Mater. Sci. 122, 183-190. doi:10.1016/j.commatsci.2016.05.020

Maynadiè, J., Salant, A., Falqui, A., Respaud, M., Shaviv, E., Banin, U., et al. (2009). Cobalt growth on the tips of CdSe nanorods. Angew. Chem. Int. Ed. 48, 1814-1817. doi:10.1002/anie.200804798

McDaniel, H., and Shim, M. (2009). Size and growth rate dependent structural diversification of $\mathrm{Fe}_{3} \mathrm{O}_{4} / \mathrm{CdS}$ anisotropic nanocrystal heterostructures. ACS Nano 3, 434-440. doi:10.1021/nn800737a

Melinon, P., Begin-Colin, S., Duvail, J. L., Gauffre, F., Boime, N. H., Ledoux, G., et al. (2014). Engineered inorganic core/shell nanoparticles. Phys. Rep. 543, 163-197. doi:10.1016/j.physrep.2014.05.003

Menagen, G., Mocatta, D., Salant, A., Popov, I., Dorfs, D., and Banin, U. (2008). Selective gold growth on CdSe seeded CdS nanorods. Chem. Mater. 20, 6900-6902. doi:10.1021/cm801702x

Michalet, X., Pinaud, F. F., Bentolila, L. A., Tsay, J. M., Doose, S., Li, J. J., et al. (2005). Quantum dots for live cells, in vivo imaging, and diagnostics. Science 307, 538-544. doi:10.1126/science.1104274

Midgley, P. A., and Weyland, M. (2003). 3D electron microscopy in the physical sciences: the development of Z-contrast and EFTEM tomography. Ultramicroscopy 96, 413-431. doi:10.1016/S0304-3991(03)00105-0

Mirtchev, P., Liao, K., Jaluague, E., Qiao, Q., Tian, Y., Varela, M., et al. (2014). $\mathrm{Fe}_{2} \mathrm{O}_{3} / \mathrm{Cu}_{2} \mathrm{O}$ heterostructured nanocrystals. J. Mater. Chem. A 2, 8525-8533. doi:10.1039/c4ta01757a

Mokari, T., Rothenberg, E., Popov, I., Costi, R., and Banin, U. (2004). Selective growth of metal tips onto semiconductor quantum rods and tetrapods. Science 304, 1787-1790. doi:10.1126/science.1097830

Mokari, T., Sztrum, C. G., Salant, A., Rabani, E., and Banin, U. (2005). Formation of asymmetric one-sided metal-tipped semiconductor nanocrystal dots and rods. Nat. Mater. 4, 855-863. doi:10.1038/nmat1505

Moon, G. D., Ko, S., Min, Y., Zeng, J., Xia, Y., and Jeong, U. (2011). Chemical transformations of nanostructured materials. Nano Today 6, 186-203. doi:10.1016/j.nantod.2011.02.006

Nag, A., Kundu, J., and Hazarika, A. (2014). Seeded-growth, nanocrystal-fusion, ion-exchange and inorganic-ligand mediated formation of semiconductorbased colloidal heterostructured nanocrystals. CrystEngComm 16, 9391-9407. doi:10.1039/c4ce00462k

Nakhjavan, B., Tahir, M. N., Natalio, F., Gao, H., Schneider, K., Schladt,

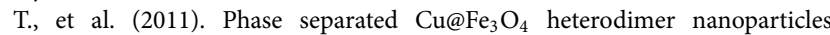
from organometallic reactants. J. Mater. Chem. 21, 8605-8611. doi:10.1039/ cljm10922g

Nakibli, Y., and Amirav, L. (2016). Selective growth of Ni tips on nanorod photocatalysts. Chem. Mater. 28, 4524-4527. doi:10.1021/acs.chemmater.6b01482

Narayan, J., and Larson, B. C. (2003). Domain epitaxy: a unified paradigm for thin film growth. J. Appl. Phys. 93, 278-285. doi:10.1063/1.1528301

Niederberger, M., and Pinna, N. (2009). Metal Oxide Nanoparticles in Organic Solvents: Synthesis, Formation, Assembly and Application. London, UK: SpringerVerlag.

Niu, M., Cheng, Y., Wang, Y., Cui, L., Bao, F., and Zhou, L. (2008). Novel nanocrystal heterostructures: crystallographic-oriented growth of $\mathrm{SnO}_{2}$ nanorods onto $\alpha-\mathrm{Fe}_{2} \mathrm{O}_{3}$ nanohexahedron. Cryst. Growth Des. 8, 1727-1729. doi:10.1021/ cg701048d

Niu, M., Huang, F., Cui, L., Huang, P., Yu, Y., and Wang, Y. (2010). Hydrothermal synthesis, structural characteristics, and enhanced photocatalysis of $\mathrm{SnO}_{2} / \alpha-$ $\mathrm{Fe}_{2} \mathrm{O}_{3}$ semiconductor nanoheterostructures. ACS Nano 4, 681-688. doi:10.1021/ nn901119a

Nolle, D., Goering, E., Tietze, T., Schutz, G., Figuerola, A., and Manna, L. (2009). Structural and magnetic deconvolution of $\mathrm{FePt} / \mathrm{FeO}_{\mathrm{x}}$-nanoparticles using $\mathrm{X}$-ray magnetic circular dichroism. New J. Phys. 11, 033034. doi:10.1088/1367-2630/ $11 / 3 / 033034$

Oszajca, M. F., Bodnarchuk, M. I., and Kovalenko, M. V. (2014). Precisely engineered colloidal nanoparticles and nanocrystals for $\mathrm{Li}$-Ion and $\mathrm{Na}$-Ion batteries model systems or practical solutions? Chem. Mater. 26, 5422-5432. doi:10.1021/ cm5024508

Palui, G., Aldeek, F., Wang, W., and Mattoussi, H. (2015). Strategies for interfacing inorganic nanocrystals with biological systems based on polymer-coating. Chem. Soc. Rev. 44, 193-227. doi:10.1039/c4cs00124a 
Pan, Y., Gao, J. H., Zhang, B., Zhang, X. X., and Xu, B. (2010). Colloidosome-based synthesis of a multifunctional nanostructure of silver and hollow iron oxide nanoparticles. Langmuir 26, 4184-4187. doi:10.1021/la904067q

Pang, X., Wan, C., Wang, M., and Lin, Z. (2014). Strictly biphasic soft and hard janus structures: synthesis, properties, and applications. Angew. Chem. Int. Ed. 53, 5524-5538. doi:10.1002/anie.201309352

Parak, W. J., Gerion, D., Pellegrino, T., Zanchet, D., Micheel, C., Williams, S. C., et al. (2003). Biological applications of colloidal nanocrystals. Nanotechnology 14, R15-R27. doi:10.1088/0957-4484/14/7/201

Park, J., Joo, J., Kwon, S. G., Jang, Y., and Hyeon, T. (2007). Synthesis of monodisperse spherical nanocrystals. Angew. Chem. Int. Ed. Engl. 46, 4630-4660. doi:10.1002/anie.200603148

Pavlopoulos, N. G., Dubose, J. T., Pinna, N., Willinger, M.-G., Char, K., and Pyun, J. (2016). Synthesis and assembly of dipolar heterostructured tetrapods: colloidal polymers with "Giant tert-butyl" groups. Angew. Chem. Int. Ed. 55, 1787-1791. doi:10.1002/anie.201510458

Pazos-Perez, N., Gao, Y., Hilgendorff, M., Irsen, S., Perez-Juste, J., Spasova, M., et al. (2007). Magnetic-noble metal nanocomposites with morphology-dependent optical response. Chem. Mater. 19, 4415-4422. doi:10.1021/cm070248o

Pellegrino, T., Fiore, A., Carlino, E., Giannini, C., Cozzoli, P. D., Ciccarella, G., et al. (2006). Heterodimers based on $\mathrm{CoPt}_{3}$-Au nanocrystals with tunable domain size. J. Am. Chem. Soc. 128, 6690-6698. doi:10.1021/ja0607741

Peng, S., Lei, C., Ren, Y., Cook, R. E., and Sun, Y. (2011). Plasmonic/magnetic bifunctional nanoparticles. Angew. Chem. Int. Ed. 50, 3158-3163. doi:10.1002/ anie.201007794

Peng, X., Chen, J., Misewich, J. A., and Wong, S. S. (2009). Carbon nanotubenanocrystal heterostructures. Chem. Soc. Rev. 38, 1076-1098. doi:10.1039/ b811424m

Pineider, F., De Julián Fernández, C., Videtta, V., Carlino, E., Al Hourani, A., Wilhelm, F., et al. (2013). Spin-polarization transfer in colloidal magneticplasmonic Au/iron oxide hetero-nanocrystals. ACS Nano 7, 857-866. doi: $10.1021 / \mathrm{nn} 305459 \mathrm{~m}$

Purbia, R., and Paria, S. (2015). Yolk/shell nanoparticles: classifications, synthesis, properties, and applications. Nanoscale 7, 19789-19873. doi:10.1039/ c5nr04729c

Qi, J., Lai, X., Wang, J., Tang, H., Ren, H., Yang, Y., et al. (2015). Multi-shelled hollow micro-/nanostructures. Chem. Soc. Rev. 44, 6749-6773. doi:10.1039/c5cs00344j

Quarta, A., Di Corato, R., Manna, L., Ragusa, A., and Pellegrino, T. (2007). Fluorescent-magnetic hybrid nanostructures: preparation, properties, and applications in biology. IEEE Trans. Nanobioscience 6, 298-308. doi:10.1109/TNB. 2007.908989

Randle, V. (1997). The Role of the Coincidence Site Lattice in Grain Boundary Engineering. Cambridge, England: Woodhead Publishing Limited.

Rawalekar, S., and Mokari, T. (2013). Rational design of hybrid nanostructures for advanced photocatalysis. Adv. Energy Mater. 3, 12-27. doi:10.1002/aenm. 201200511

Ringe, E. (2014). Nanocrystalline materials: recent advances in crystallographic characterization techniques. IUCrJ 1, 530-539. doi:10.1107/ S2052252514020818

Ross, F. M. (2015). Opportunities and challenges in liquid cell electron microscopy. Science 350, 1490. doi:10.1126/science.aaa9886

Ruckenstein, E., and Djikaev, Y. S. (2005). Recent developments in the kinetic theory of nucleation. Adv. Colloid Interface Sci. 118, 51-72. doi:10.1016/j.cis. 2005.06.001

Sadowski, T., and Ramprasad, R. (2010). Ab initio thermodynamic model to assess stability of heterostructure nanocrystals. Appl. Phys. Lett. 96, 101906. doi:10.1063/1.3330924

Schick, I., Lorenz, S., Gehrig, D., Tenzer, S., Storck, W., Fischer, K., et al. (2014). Inorganic Janus particles for biomedical applications. Beilstein J. Nanotechnol. 5, 2346-2362. doi:10.3762/bjnano.5.244

Schladt, T. D., Graf, T., Köhler, O., Bauer, H., Dietzsch, M., Mertins, J., et al. (2012). Synthesis and magnetic properties of FePt@MnO nano-heteroparticles. Chem. Mater. 24, 525-535. doi:10.1021/cm2030685

Schladt, T. D., Shukoor, M. I., Schneider, K., Tahir, M. N., Natalio, F., Ament, I., et al. (2010). Au@MnO nanoflowers: hybrid nanocomposites for selective dual functionalization and imaging. Angew. Chem. Int. Ed. 49, 3976-3980. doi:10. 1002/anie.200906689

Selvan, S. T., Patra, P. K., Ang, C. Y., and Ying, J. Y. (2007). Synthesis of silica-coated semiconductor and magnetic quantum dots and their use in the imaging of live cells. Angew. Chem. Int. Ed. 46, 2448-2452. doi:10.1002/anie.200604245
Seyring, M., Song, X., and Rettenmayr, M. (2011). Advance in orientation microscopy: quantitative analysis of nanocrystalline structures. ACS Nano 5, 2580-2586. doi:10.1021/nn1023126

Shi, W., Sahoo, Y., Zeng, H., Ding, Y., Swihart, M. T., and Prasad, P. N. (2006a). Anisotropic growth of PbSe nanocrystals on $\mathrm{Au}-\mathrm{Fe}_{3} \mathrm{O}_{4}$ hybrid nanoparticles. Adv. Mater. 18, 1889-1894. doi:10.1002/adma.200600685

Shi, W., Zeng, H., Sahoo, Y., Ohulchanskyy, T. Y., Ding, Y., Wang, Z. L., et al. (2006b). A general approach to binary and ternary hybrid nanocrystals. Nano Lett. 6, 875-881. doi:10.1021/nl0600833

Shim, M., and McDaniel, H. (2010). Anisotropic nanocrystal heterostructures: synthesis and lattice strain. Curr. Opin. Solid State Mater. Sci. 14, 83-94. doi:10.1016/j.cossms.2010.04.001

Sitt, A., Hadar, I., and Banin, U. (2013). Band-gap engineering, optoelectronic properties and applications of colloidal heterostructured semiconductor nanorods. Nano Today 8, 494-513. doi:10.1016/j.nantod.2013.08.002

Slaton, R. D., Bae, I.-T., Lutz, P. S., Pathade, L., and Maye, M. M. (2015). The transformation of $\alpha$-Fe nanoparticles into multi-domain $\mathrm{FeNi}-\mathrm{M}_{3} \mathrm{O}_{4}(\mathrm{M}=\mathrm{Fe}$, Ni) heterostructures by galvanic exchange. J. Mater. Chem. C 3, 6367-6375. doi:10.1039/c5tc00929d

Song, H. (2015). Metal hybrid nanoparticles for catalytic organic and photochemical transformations. Acc. Chem. Res. 48, 491-499. doi:10.1021/ar500411s

Steiner, D., Mokari, T., Banin, U., and Millo, O. (2005). Electronic structure of metal-semiconductor nanojunctions in gold CdSe nanodumbbells. Phys. Rev. Lett. 95, 056805. doi:10.1103/PhysRevLett.95.056805

Su, L. W., Jing, Y., and Zhou, Z. (2011). Li ion battery materials with core-shell nanostructures. Nanoscale 3, 3967-3983. doi:10.1039/c1nr10550g

Sun, X., Guo, S., Liu, Y., and Sun, S. (2012). Dumbbell-like PtPd-Fe $\mathrm{O}_{4}$ nanoparticles for enhanced electrochemical detection of $\mathrm{H}_{2} \mathrm{O}_{2}$. Nano Lett. 12, 4859-4863. doi:10.1021/nl302358e

Talapin, D. V., Lee, J. S., Kovalenko, M. V., and Shevchenko, E. V. (2010). Prospects of colloidal nanocrystals for electronic and optoelectronic applications. Chem. Rev. 110, 389-458. doi:10.1021/cr900137k

Tao, K., Zhou, H., Dou, H., Xing, B., Li, W., and Sun, K. (2009). Direct deposition of fluorescent emission-tunable CdSe on magnetite nanocrystals. J. Phys. Chem. C 113, 8762-8766. doi:10.1021/jp901335s

Teranishi, T., Inoue, Y., Nakaya, M., Oumi, Y., and Sano, T. (2004). Nanoacorns: anisotropically phase-segregated CoPd sulfide nanoparticles. J. Am. Chem. Soc 126, 9914-9915. doi:10.1021/ja047606y

Teranishi, T., Inoue, Y., Saruyama, M., Nakaya, M., and Kanehara, M. (2007a). Anisotropically phase-segregated $\mathrm{Co}_{9} \mathrm{~S}_{8} / \mathrm{PdS}_{\mathrm{x}}$ nanoacorns: stability improvement and new heterostructures. Chem. Lett. 36, 490-491. doi:10.1246/cl.2007.490

Teranishi, T., Saruyama, M., Nakaya, M., and Kanehara, M. (2007b). Anisotropically phase-segregated Pd-Co-Pd sulfide nanoparticles formed by fusing two Co-Pd sulfide nanoparticles. Angew. Chem. Int. Ed. 46, 1713-1715. doi:10.1002/anie. 200603865

Thompson, C. V. (2012). Solid-state dewetting of thin films. Annu. Rev. Mater. Res. 42, 399-434. doi:10.1146/annurev-matsci-070511-155048

Tian, G., Zhang, X., Gu, Z., and Zhao, Y. (2015). Recent advances in upconversion nanoparticles-based multifunctional nanocomposites for combined cancer therapy. Adv. Mater. 27, 7692-7712. doi:10.1002/adma.201503280

Tong, H., Ouyang, S., Bi, Y., Umezawa, N., Oshikiri, M., and Ye, J. (2012). Nanophotocatalytic materials: possibilities and challenges. Adv. Mater. 24, 229-251. doi:10.1002/adma.201102752

Umut, E., Pineider, F., Arosio, P., Sangregorio, C., Corti, M., Tabak, F., et al. (2012). Magnetic, optical and relaxometric properties of organically coated goldmagnetite $\left(\mathrm{Au}-\mathrm{Fe}_{3} \mathrm{O}_{4}\right)$ hybrid nanoparticles for potential use in biomedical applications. J. Magn. Magn. Mater. 324, 2373-2379. doi:10.1016/j.jmmm.2012. 03.005

Vaneski, A., Schneider, J., Susha, A. S., and Rogach, A. L. (2014). Colloidal hybrid heterostructures based on II-VI semiconductor nanocrystals for photocatalytic hydrogen generation. J. Photochem. Photobiol. C Photochem. Rev. 19, 52-61. doi:10.1016/j.jphotochemrev.2013.12.001

Vanmaekelbergh, D. (2011). Self-assembly of colloidal nanocrystals as route to novel classes of nanostructured materials. Nano Today 6, 419-437. doi:10.1016/ j.nantod.2011.06.005

Velasco, V., Munoz, L., Mazario, E., Menendez, N., Herrasti, P., Hernando, A., et al. (2015). Chemically synthesized $\mathrm{Au}-\mathrm{Fe}_{3} \mathrm{O}_{4}$ nanostructures with controlled optical and magnetic properties. J. Phys. D Appl. Phys. 48, 035502. doi:10.1088/ 0022-3727/48/3/035502 
Victor, V., Laura, M., Eva, M., Nieves, M., Pilar, H., Antonio, H., et al. (2015). Chemically synthesized $\mathrm{Au}-\mathrm{Fe}_{3} \mathrm{O}_{4}$ nanostructures with controlled optical and magnetic properties. J. Phys. D Appl. Phys. 48, 035502. doi:10.1088/0022-3727/ 48/3/035502

Vogel, N., Retsch, M., Fustin, C. A., Del Campo, A., and Jonas, U. (2015). Advances in colloidal assembly: the design of structure and hierarchy in two and three dimensions. Chem. Rev. 115, 6265-6311. doi:10.1021/cr400081d

Wang, C., Daimon, H., and Sun, S. (2009a). Dumbbell-like Pt- $\mathrm{Fe}_{3} \mathrm{O}_{4}$ nanoparticles and their enhanced catalysis for oxygen reduction reaction. Nano Lett. 9, 1493-1496. doi:10.1021/nl8034724

Wang, C., Wei, Y., Jiang, H., and Sun, S. (2009b). Tug-of-War in nanoparticles: competitive growth of $\mathrm{Au}$ on $\mathrm{Au}-\mathrm{Fe}_{3} \mathrm{O}_{4}$ nanoparticles. Nano Lett. 9, 4544-4547. doi:10.1021/nl903077t

Wang, C., Xu, C., Zeng, H., and Sun, S. (2009c). Recent progress in syntheses and applications of dumbbell-like nanoparticles. Adv. Mater. 21, 3045-3052. doi:10.1002/adma.200900320

Wang, L., Liu, X. Z., Yang, M., Fan, Y. J., and Zhan, J. H. (2009d). Electroless plating of nickel nanoparticles on CdS nanowires. Eur. J. Inorg. Chem. 897-902. doi:10.1002/ejic.200800855

Wang, C., Yin, H., Dai, S., and Sun, S. (2010). A general approach to noble metalmetal oxide dumbbell nanoparticles and their catalytic application for CO oxidation. Chem. Mater. 22, 3277-3282. doi:10.1021/cm100603r

Wang, D., Li, X., Li, H., Li, L., Hong, X., Peng, Q., et al. (2013a). Semiconductornoble metal hybrid nanomaterials with controlled structures. J. Mater. Chem. A 1, 1587-1590. doi:10.1039/c2ta00765g

Wang, W. S., Dahl, M., and Yin, Y. D. (2013b). Hollow nanocrystals through the nanoscale Kirkendall effect. Chem. Mater. 25, 1179-1189. doi:10.1021/ cm3030928

Wang, D., and Li, Y. (2010). One-pot protocol for Au-based hybrid magnetic nanostructures via a noble-metal-induced reduction process. J. Am. Chem. Soc. 132, 6280-6281. doi:10.1021/ja100845v

Wang, F., Richards, V. N., Shields, S. P., and Buhro, W. E. (2014). Kinetics and mechanisms of aggregative nanocrystal growth. Chem. Mater. 26, 5-21. doi:10.1021/cm402139r

Wang, L., Bai, J., Li, Y., and Huang, Y. (2008). Multifunctional nanoparticles displaying magnetization and near-IR absorption. Angew. Chem. Int. Ed. Engl. 47, 2439-2442. doi:10.1002/anie.200800014

Wang, Y., He, J., Liu, C., Chong, W. H., and Chen, H. (2015). Thermodynamics versus kinetics in nanosynthesis. Angew. Chem. Int. Ed. 54, 2022-2051. doi:10.1002/anie.201402986

Wark, S. E., Hsia, C.-H., and Son, D. H. (2008). Effects of ion solvation and volume change of reaction on the equilibrium and morphology in cation-exchange reaction of nanocrystals. J. Am. Chem. Soc. 130, 9550-9555. doi:10.1021/ ja802187c

Wei, Y., Klajn, R., Pinchuk, A. O., and Grzybowski, B. A. (2008). Synthesis, shape control, and optical properties of hybrid $\mathrm{Au} / \mathrm{Fe}_{3} \mathrm{O}_{4}$ "nanoflowers". Small 4, 1635-1639. doi:10.1002/smll.200800511

Wetz, F., Soulantica, K., Falqui, A., Respaud, M., Snoeck, E., and Chaudret, B. (2007). Hybrid Co-Au nanorods: controlling Au nucleation and location. Angew. Chem. Int. Ed. Engl. 46, 7079-7081. doi:10.1002/anie.200702017

Wilker, M. B., Schnitzenbaumer, K. J., and Dukovic, G. (2012). Recent progress in photocatalysis mediated by colloidal II-VI nanocrystals. Isr. J. Chem. 52, 1002-1015. doi:10.1002/ijch.201200073

Wu, B., Zhang, H., Chen, C., Lin, S., and Zheng, N. (2009). Interfacial activation of catalytically inert $\mathrm{Au}(6.7 \mathrm{~nm})-\mathrm{Fe}_{3} \mathrm{O}_{4}$ dumbbell nanoparticles for $\mathrm{CO}$ oxidation. Nano Res. 2, 975-983. doi:10.1007/s12274-009-9102-Z

Wu, H. M., Chen, O., Zhuang, J. Q., Lynch, J., Lamontagne, D., Nagaoka, Y., et al. (2011a). Formation of heterodimer nanocrystals: $\mathrm{UO}_{2} / \mathrm{In}_{2} \mathrm{O}_{3}$ and $\mathrm{FePt} / \mathrm{In}_{2} \mathrm{O}_{3}$. J. Am. Chem. Soc. 133, 14327-14337. doi:10.1021/ja2023724

Wu, J. J., Hou, Y. L., and Gao, S. (2011b). Controlled synthesis and multifunctional properties of FePt-Au heterostructures. Nano Res. 4, 836-848. doi:10.1007/ s12274-011-0140-y

Xu, B., Zhou, G., and Wang, X. (2015). Rational synthesis and the structure-property relationships of nanoheterostructures: a combinative study of experiments and theory. NPG Asia Mater. 7, e164. doi:10.1038/am.2015.4
Xu, C., Xie, J., Ho, D., Wang, C., Kohler, N., Walsh, E. G., et al. (2008). $\mathrm{Au}^{-\mathrm{Fe}_{3} \mathrm{O}_{4}}$ dumbbell nanoparticles as dual-functional probes. Angew. Chem. Int. Ed. 47, 173-176. doi:10.1002/anie.200704392

Xu, Z., Liu, Y., Ren, F., Yang, F., and Ma, D. (2016). Development of functional nanostructures and their applications in catalysis and solar cells. Coord. Chem. Rev. 32, 153-180. doi:10.1016/j.ccr.2016.03.002

Yan, J., Bae, S. C., and Granick, S. (2015a). Colloidal superstructures programmed into magnetic janus particles. Adv. Mater. 27, 874-879. doi:10.1002/adma. 201403857

Yan, Y., Miao, J., Yang, Z., Xiao, F.-X., Yang, H. B., Liu, B., et al. (2015b). Carbon nanotube catalysts: recent advances in synthesis, characterization and applications. Chem. Soc. Rev. 44, 3295-3346. doi:10.1039/c4cs00492b

Yang, J., Elim, H. I., Zhang, Q., Lee, J. Y., and Ji, W. (2006a). Rational synthesis, self-assembly, and optical properties of $\mathrm{PbS}$-Au heterogeneous nanostructures via preferential deposition. J. Am. Chem. Soc. 128, 11921-11926. doi:10.1021/ ja062494r

Yang, J., Levina, L., Sargent, E. H., and Kelley, S. O. (2006b). Heterogeneous deposition of noble metals on semiconductor nanoparticles in organic or aqueous solvents. J. Mater. Chem. 16, 4025-4028. doi:10.1039/b612255h

Yang, J., Peng, J., Zhang, Q., Peng, F., Wang, H., and Yu, H. (2009). One-step synthesis and characterization of gold-hollow PbSx hybrid nanoparticles. Angew. Chem. Int. Ed. 48, 3991-3995. doi:10.1002/anie.200806036

Yang, J., Son, J. S., Yu, J. H., Joo, J., and Hyeon, T. (2013). Advances in the colloidal synthesis of two-dimensional semiconductor nanoribbons. Chem. Mater. 25, 1190-1198. doi:10.1021/cm303145f

Yang, W., Lei, W., Yu, Y., Zhu, W., George, T. A., Li, X. Z., et al. (2015). From FePt- $\mathrm{Fe}_{3} \mathrm{O}_{4}$ to $\mathrm{L}_{0}-\mathrm{FePt}-\mathrm{Fe}$ nanocomposite magnets with a gradient interface. J. Mater. Chem. C 3, 7075-7080. doi:10.1039/c5tc01145k

Yang, J., and Ying, J. Y. (2009). A general phase-transfer protocol for metal ions and its application in nanocrystal synthesis. Nat. Mater. 8, 683-689. doi:10.1038/ nmat 2490

Yin, H., Wang, C., Zhu, H., Overbury, S. H., Sun, S., and Dai, S. (2008). Colloidal deposition synthesis of supported gold nanocatalysts based on $\mathrm{Au}-\mathrm{Fe}_{3} \mathrm{O}_{4}$ dumbbell nanoparticles. Chem. Commun. 4357-4359. doi:10.1039/b807591c

Yin, P. T., Shah, S., Chhowalla, M., and Lee, K.-B. (2015). Design, synthesis, and characterization of graphene-nanoparticle hybrid materials for bioapplications. Chem. Rev. 115, 2483-2531. doi:10.1021/cr500537t

Yu, H., Chen, M., Rice, P. M., Wang, S. X., White, R. L., and Sun, S. (2005). Dumbbell-like bifunctional $\mathrm{Au}-\mathrm{Fe}_{3} \mathrm{O}_{4}$ nanoparticles. Nano Lett. 5, 379-382. doi:10.1021/nl047955q

Yu, S. M., Hachtel, J. A., Chisholm, M. F., Pantelides, S. T., Laromaine, A., and Roig, A. (2015). Magnetic gold nanotriangles by microwave-assisted polyol synthesis. Nanoscale 7, 14039-14046. doi:10.1039/c5nr03113c

Yuhas, B. D., Habas, S. E., Fakra, S. C., and Mokari, T. (2009). Probing compositional variation within hybrid nanostructures. ACS Nano 3, 3369-3376. doi:10.1021/ nn901107p

Zanella, M., Falqui, A., Kudera, S., Manna, L., Casula, M. F., and Parak, W. J. (2008) Growth of colloidal nanoparticles of group II-VI and IV-VI semiconductors on top of magnetic iron-platinum nanocrystals. J. Mater. Chem. 18, 4311-4317. doi:10.1039/b804154g

Zeng, J., Jianliu, H., Liu, C., Wu, C. H., Lin, Y., Wang, X., et al. (2010). Gold-based hybrid nanocrystals through heterogeneous nucleation and growth. Adv. Mater. 22, 1936-1940. doi:10.1002/adma.200903982

Zhai, Y., Han, L., Wang, P., Li, G., Ren, W., Liu, L., et al. (2011). Superparamagnetic plasmonic nanohybrids: shape-controlled synthesis, TEM-induced structure evolution, and efficient sunlight-driven inactivation of bacteria. ACS Nano 5, 8562-8570. doi:10.1021/nn201875k

Zhang, H., Liu, Y., Yao, D., and Yang, B. (2012). Hybridization of inorganic nanoparticles and polymers to create regular and reversible self-assembly architectures Chem. Soc. Rev. 41, 6066-6088. doi:10.1039/c2cs35038f

Zhang, J., Huang, F., and Lin, Z. (2010). Progress of nanocrystalline growth kinetics based on oriented attachment. Nanoscale 2, 18-34. doi:10.1039/b9nr00047j

Zhang, L., Dou, Y. H., and Gu, H. C. (2006). Synthesis of $\mathrm{Ag}-\mathrm{Fe}_{3} \mathrm{O}_{4}$ heterodimeric nanoparticles. J. Colloid Interface Sci. 297, 660-664. doi:10.1016/j. jcis.2005.11.009 
Zhang, Q., Wang, W., Goebl, J., and Yin, Y. (2009). Self-templated synthesis of hollow nanostructures. Nano Today 4, 494-507. doi:10.1016/j.nantod.2009. 10.008

Zhou, W., Cheng, C., Liu, J., Tay, Y. Y., Jiang, J., Jia, X., et al. (2011). Epitaxial growth of branched $\alpha-\mathrm{Fe}_{2} \mathrm{O}_{3} / \mathrm{SnO}_{2}$ nano-heterostructures with improved lithium-ion battery performance. Adv. Func. Mater. 21, 2439-2445. doi:10.1002/ adfm.201100088

Zhu, J., Wu, J., Liu, F., Xing, R., Zhang, C., Yang, C., et al. (2013). Controlled synthesis of FePt-Au hybrid nanoparticles triggered by reaction atmosphere and FePt seeds. Nanoscale 5, 9141-9149. doi:10.1039/c3nr02911e
Conflict of Interest Statement: The authors declare that the research was conducted in the absence of any commercial or financial relationships that could be construed as a potential conflict of interest.

Copyright (c) 2016 Scarfiello, Nobile and Cozzoli. This is an open-access article distributed under the terms of the Creative Commons Attribution License (CC BY). The use, distribution or reproduction in other forums is permitted, provided the original author(s) or licensor are credited and that the original publication in this journal is cited, in accordance with accepted academic practice. No use, distribution or reproduction is permitted which does not comply with these terms. 